\title{
The vaginal microbiome and preterm birth
}

\author{
Jennifer M. Fettweis ${ }^{1,2,3}$, Myrna G. Serrano ${ }^{1,3}$, J. Paul Brooks ${ }^{3,4}$, David J. Edwards ${ }^{3,5}$, \\ Philippe H. Girerd ${ }^{2,3}$, Hardik I. Parikh', Bernice Huang', Tom J. Arodz ${ }^{3,6}$, Laahirie Edupuganti, ${ }^{1,3}$,

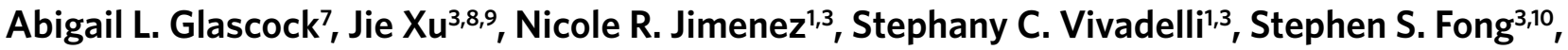 \\ Nihar U. Sheth"1, Sophonie Jean1, Vladimir Lee ${ }^{1,3}$, Yahya A. Bokhari6, Ana M. Lara1, Shreni D. Mistry1, \\ Robert A. Duckworth III', Steven P. Bradley1, Vishal N. Koparde ${ }^{11}$, X. Valentine Orenda ${ }^{1}$, \\ Sarah H. Milton², Sarah K. Rozycki'2, Andrey V. Matveyev', Michelle L. Wright ${ }^{13,14,15}$, \\ Snehalata V. Huzurbazar ${ }^{16}$, Eugenie M. Jackson ${ }^{16}$, Ekaterina Smirnova ${ }^{17,18}{ }^{18}$ Jonas Korlach ${ }^{19}$, \\ Yu-Chih Tsai ${ }^{19}$, Molly R. Dickinson', Jamie L. Brooks', Jennifer I. Drake', Donald O. Chaffin ${ }^{20}$, \\ Amber L. Sexton ${ }^{20}$, Michael G. Gravett ${ }^{20,21}$, Craig E. Rubens ${ }^{20}$, N. Romesh Wijesooriya9 \\ Karen D. Hendricks-Muñoz ${ }^{3,8,9}$, Kimberly K. Jefferson ${ }^{1,3}$, Jerome F. Strauss III ${ }^{2,3}$ and Gregory A. Buck ${ }^{1,3,6 \star}$
}

The incidence of preterm birth exceeds $10 \%$ worldwide. There are significant disparities in the frequency of preterm birth among populations within countries, and women of African ancestry disproportionately bear the burden of risk in the United States. In the present study, we report a community resource that includes 'omics' data from approximately 12,000 samples as part of the integrative Human Microbiome Project. Longitudinal analyses of 16 S ribosomal RNA, metagenomic, metatranscriptomic and cytokine profiles from 45 preterm and 90 term birth controls identified harbingers of preterm birth in this cohort of women predominantly of African ancestry. Women who delivered preterm exhibited significantly lower vaginal levels of Lactobacillus crispatus and higher levels of BVAB1, Sneathia amnii, TM7-H1, a group of Prevotella species and nine additional taxa. The first representative genomes of BVAB1 and TM7-H1 are described. Preterm-birth-associated taxa were correlated with proinflammatory cytokines in vaginal fluid. These findings highlight new opportunities for assessment of the risk of preterm birth.

A pproximately 15 million preterm births at less than 37 weeks of gestation occur annually worldwide ${ }^{1}$. Preterm birth (PTB) remains the second most common cause of neonatal death across the globe, and the most common cause of infant mortality in middle- and high-income economies ${ }^{2}$. The consequences of PTB persist from early childhood into adolescence and adulthood ${ }^{3,4}$. In the United States, striking population differences with respect to PTB exist, with women of African ancestry having a substantially larger burden of risk. The estimated annual cost of PTB in the United States alone is over US $\$ 26.2$ billion $^{5}$. Despite these statistics, there remains a paucity of effective strategies for predicting and preventing PTB.

Although maternal and fetal genetics, and gene-environment interactions, clearly play roles in determining the length of gestation, environmental factors, including the microbiome, are the most important contributors to PTB, particularly among women of African ancestry ${ }^{6}$. Microbe-induced inflammation resulting from urinary tract infection, sexually transmitted infections, including trichomoniasis, or bacterial vaginosis is thought to be a cause of $\mathrm{PTB}^{7,8}$. Ascension of microbes ${ }^{7,9}$ from the lower reproductive tract to the placenta, fetal membranes and uterine cavity, and hematogenous spread of periodontal pathogens from the mouth, have also been invoked to explain the up to $40-50 \%$ of preterm births that are associated with microbial etiologies ${ }^{10,1}$

A homogeneous Lactobacillus-dominated microbiome has long been considered the hallmark of health in the female reproductive tract. In contrast, a vaginal microbiome with high species diversity, as observed with bacterial vaginosis, has been associated with increased risk for acquisition and transmission of sexually

'Department of Microbiology and Immunology, School of Medicine, Virginia Commonwealth University, Richmond, VA, USA. ${ }^{2}$ Department of Obstetrics and Gynecology, School of Medicine, Virginia Commonwealth University, Richmond, VA, USA. ${ }^{3}$ Center for Microbiome Engineering and Data Analysis, Virginia Commonwealth University, Richmond, VA, USA. ${ }^{4}$ Supply Chain Management and Analytics, School of Business, Virginia Commonwealth University, Richmond, VA, USA. ${ }^{5}$ Department of Statistical Sciences and Operations Research, College of Humanities and Sciences, Virginia Commonwealth University, Richmond, VA, USA. ${ }^{6}$ Department of Computer Science, College of Engineering, Virginia Commonwealth University, Richmond, VA, USA. ${ }^{7}$ VCU Life Sciences, Virginia Commonwealth University, Richmond, VA, USA. ${ }^{8}$ Division of Neonatal Medicine, School of Medicine, Virginia Commonwealth University, Richmond, VA, USA. ' Department of Pediatrics, School of Medicine, Children's Hospital of Richmond at Virginia Commonwealth University, Richmond, VA, USA. ${ }^{10}$ Department of Chemical and Life Science Engineering, College of Engineering, Virginia Commonwealth University, Richmond, VA, USA. "'Center for the Study of Biological Complexity, VCU Life Sciences, Virginia Commonwealth University, Richmond, VA, USA. ${ }^{12}$ School of Medicine, Virginia Commonwealth University, Richmond, VA, USA. ${ }^{13}$ Nell Hodgson Woodruff School of Nursing, Emory University, Atlanta, GA, USA. ${ }^{14}$ Department of Women's Health, Dell School of Medicine, University of Texas at Austin, Austin, TX, USA. ${ }^{15}$ School of Nursing, University of Texas at Austin, Austin, TX, USA. ${ }^{16}$ Department of Biostatistics, School of Public Health, West Virginia University, Morgantown, WV, USA. ${ }^{17}$ Department of Mathematical Sciences, University of Montana, Missoula, MT, USA. ${ }^{18}$ Department of Biostatistics, School of Medicine, Virginia Commonwealth University, Richmond, VA, USA. ${ }^{19}$ Pacific Biosciences, Menlo Park, CA, USA. ${ }^{20}$ Global Alliance to Prevent Prematurity and Stillbirth, Seattle, WA, USA. ${ }^{21}$ Department of Obstetrics \& Gynecology, University of Washington, Seattle, WA, USA. *e-mail: gregory.buck@vcuhealth.org 
transmitted infections, PTB and pelvic inflammatory disease ${ }^{12-15}$. However, many asymptomatic healthy women have diverse vaginal microbiota. More refined approaches are needed to assess risk, promote health, and prevent and treat disease ${ }^{16-21}$.

Recent reports of the microbiome in pregnant women ${ }^{22-39}$ have suggested that the composition of the vaginal microbiome has a significant population-specific impact on PTB risk. Several studies that focused on populations predominantly of European descent ${ }^{22-25}$ have associated Lactobacillus crispatus with a lower risk of PTB, and the finding was replicated in a cohort of predominantly African descent $^{25}$. As first reported by Ravel et al. ${ }^{16}$, and subsequently confirmed in other studies ${ }^{21,40}$, the vaginal microbiome profiles of women of African and European ancestry differ significantly. Although distinct taxa have been associated with PTB in women of African ancestry in some studies ${ }^{25,26}$, others have not found significant associations ${ }^{27,30}$. Women of African descent are less likely to exhibit vaginal lactobacilli, frequently have vaginal L. crispatus predominance and are more likely to exhibit increased vaginal microbial diversity ${ }^{16,21}$. Consequently, population-specific studies may be required to assess the broad impacts of the vaginal microbiome on risk of PTB and to identify contributing taxa that may be carried by only a small subset of women.

In the present study, we report a community resource that includes samples collected longitudinally during 1,572 pregnancies of women from diverse ancestries, and omics data generated from samples collected from 597 pregnancies in a collaborative effort under the umbrella of the National Institutes of Health's integrative Human Microbiome Project (iHMP) ${ }^{41}$. Furthermore, we provided an analysis of the longitudinal, comprehensive, multi-omic profiling of vaginal samples from 45 women who experienced spontaneous PTB and 90 case-matched controls, in a cohort of women of predominantly African ancestry. In an initial analysis of this dataset, which represents one of the largest and most comprehensive studies of the vaginal microbiome to date, we identified vaginal microbial signatures in women who went on to experience PTB.

\section{Results}

The Multi-Omic Microbiome Study: Pregnancy Initiative. The longitudinal iHMP study, the Multi-Omic Microbiome Study: Pregnancy Initiative (MOMS-PI) includes a total of 1,572 pregnancies, with 992 pregnancies from clinics associated with the Research Alliance for Microbiome Science (RAMS) Registry, based at Virginia Commonwealth University (VCU) in Virginia, and 580 pregnancies from sites associated with the Global Alliance to Prevent Prematurity and Stillbirth (GAPPS) in Washington State. The resource features two comprehensive datasets of integrated microbiome and host functional properties measured longitudinally in pregnancy and the perinatal period (Fig. 1): (1) the MOMS-PI Preterm Birth (PTB) study dataset generated from a case-control study of 45 women predominantly of African ancestry, who delivered spontaneously preterm, and 90 case-matched women who delivered at term; and (2) the MOMS-PI Term Birth (TB) study dataset generated from an ethnically diverse retrospective cohort study of 90 women, who delivered at term or early term ${ }^{42}$. From a selection of 12,039 samples from 597 pregnancies, we generated: (1) 16S ribosomal RNA (rRNA) taxonomic profiles from 6,452 samples from pregnant women and 2,753 samples from neonates; (2) metagenome profiles from 930 samples from pregnant women and 146 samples from neonates; (3) metatranscriptome profiles from 297 samples from pregnant women; (4) cytokine profiles from 1,223 samples from pregnant women and 173 samples from neonates; and (5) lipid profiles from 63 samples from pregnant women. In the overall MOMS-PI study, we collected a total of 206,437 samples from pregnant women and their neonates, which have been archived in the RAMS Registry (see ramsregistry.vcu.edu) (Fig. 1c). Comprehensive health history and outcome data were also collected longitudinally.
Vaginal microbiome profiles show PTB-associated trends. In the present study, we focus our analysis on a comprehensive multi-omic profiling of vaginal samples in the MOMS-PI PTB study. We analyzed 45 single gestation pregnancies that met the criteria for spontaneous PTB (23-36 weeks 6 days of gestational age) and 90 single gestation pregnancies that extended through term ( $\geq 39$ weeks) to avoid issues possibly associated with early term births ${ }^{43-45}$. The TB controls in the MOMS-PI PTB study were case matched to the PTB group (2TB:1PTB) for age, race and annual household income. On average, the earliest samples were collected at 18 weeks of gestation, and the mean number of sampling visits per participant was 7. The respective mean and median gestational age at delivery was $34,0 / 7$ and $35,6 / 7$ for the PTB group and $40,0 / 7$ and $39,6 / 7$ for the TB group.

The cohort predominantly comprised women of African ancestry $(\sim 78 \%)$, with a median annual income of less than US\$20,000 and an average age of 26 years (Table 1, and see Supplementary Table 1). Microbiome profiles of the first vaginal samples collected at study enrollment (Fig. 2a, and see Extended Data Fig. 1) were generated by $16 \mathrm{~S}$ rRNA taxonomic analysis. For vaginal samples, the dominant bacterial taxon is one clinically meaningful measure by which to stratify samples ${ }^{16,46}$. Women who went on to deliver at term were more likely to exhibit $L$. crispatus predominance in the vaginal microbiome $(P=0.014$, Fig. 2a,b, and see Supplementary Table 2a and Extended Data Fig. 1), paralleling earlier observations ${ }^{17,22-25}$. A Markov chain analysis to assess vagitype changes throughout pregnancy did not reveal statistically significant differences in transition rates between case and control groups. However, point estimates of probabilities of transition to the BV-associated bacterium 1 (BVAB1) vagitype were higher in the PTB group, whereas point estimates of transition to the $L$. crispatus group were higher in the term group, although the differences failed to reach significance (see Supplementary Table 2b).

Overall diversity was increased in samples from women who would go on to experience PTB (see Extended Data Fig. 2), and 12 taxa showed a significant difference in abundance between the PTB and the TB groups (Fig. 2b). L. crispatus was greatly reduced in PTB samples, and several other taxa, including BVAB1, Prevotella cluster 2 and Sneathia amnii, were more abundant in PTB samples $(q<0.05$; Fig. 2b, and see Supplementary Tables 4 and 5). Prevotella cluster 2 comprises several closely related taxa of that genus ${ }^{47}$, including Prevotella timonensis and Prevotella buccalis. Through an analysis of samples collected from the 31 PTB and 59 TB subjects who had samples collected early (6-24 weeks of gestational age) in pregnancy, we identified two additional taxa that were significantly increased in PTB samples: Megasphaera type 1 and TM7-H1 (that is, BVAB-TM7) (see Extended Data Fig. 3). Both of these taxa have been previously associated with adverse conditions of vaginal health ${ }^{12}$. These findings extend those of a previous study that found carriage of BVAB1 and Sneathia species in early and mid-pregnancy to be associated with spontaneous $\mathrm{PTB}^{26}$. To our knowledge, this is the first report of an association of TM7-H1 with PTB.

Early prediction of risk for PTB is critical for the development of new strategies for prevention and intervention. As a proof of concept, we developed a model for identifying the most discriminative taxa for PTB using 16S rRNA data from samples collected at 24 weeks of gestation or earlier. Model construction involved selecting taxa that are differentially represented in the cohorts as assessed using the Mann-Whitney $U$-test (Fig. 2d, and see Extended Data Fig. 3a and Supplementary Table 5), and assigning weights to these taxa using $\mathrm{L}_{1}$-regularized logistic regression. The resulting model incorporates four taxa: S. amnii, BVAB1, Prevotella cluster 2 and TM7-H1, which are all positively correlated with PTB (Fig. 2d, and see Extended Data Fig. 3b,c). The discriminative model is significant $(P=0.0024)$ and has an expected sensitivity of $77.4 \%$, specificity of $76.3 \%$, and an area under the receiver operating characteristics (AUROC) curve 


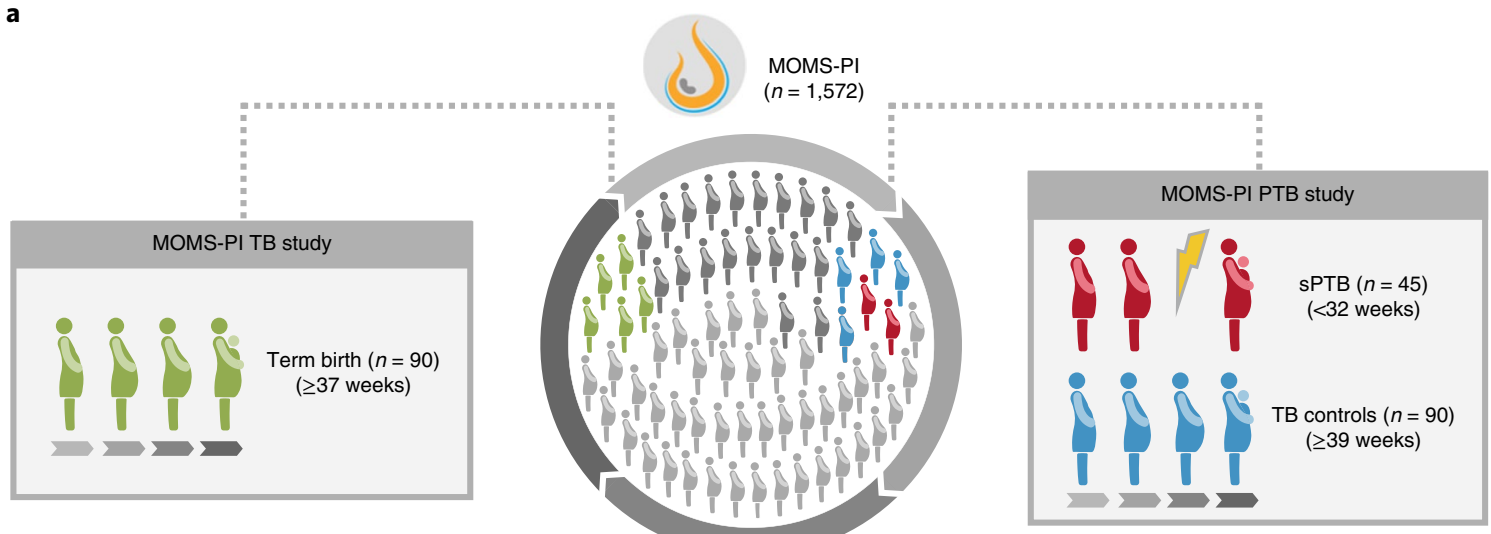

b
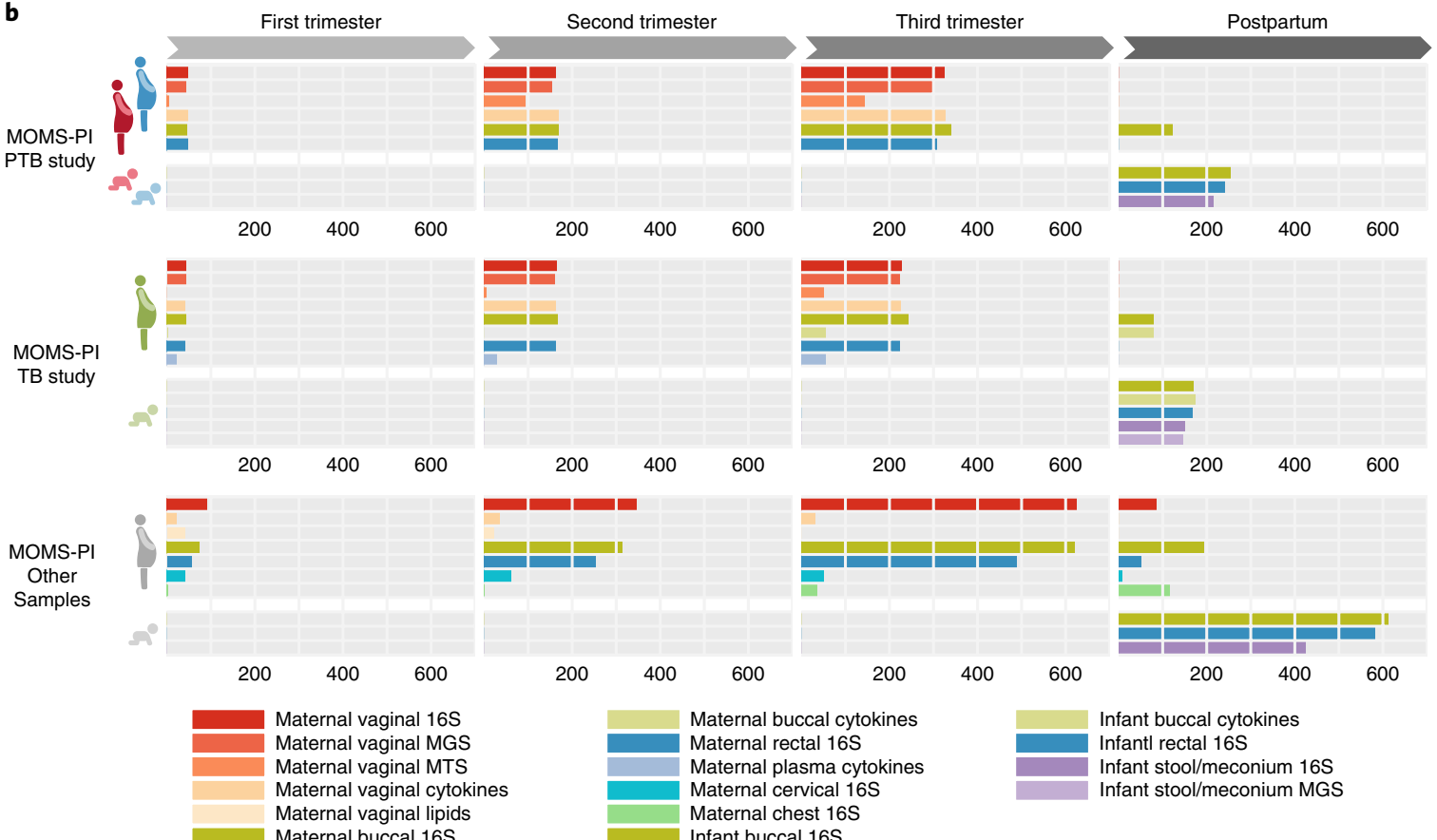

Maternal buccal cytokines

Maternal rectal $16 \mathrm{~S}$

Maternal rectal $16 \mathrm{~S}$

Maternal cervical $16 \mathrm{~S}$

Maternal chest $16 \mathrm{~S}$

Maternal buccal $16 \mathrm{~S}$
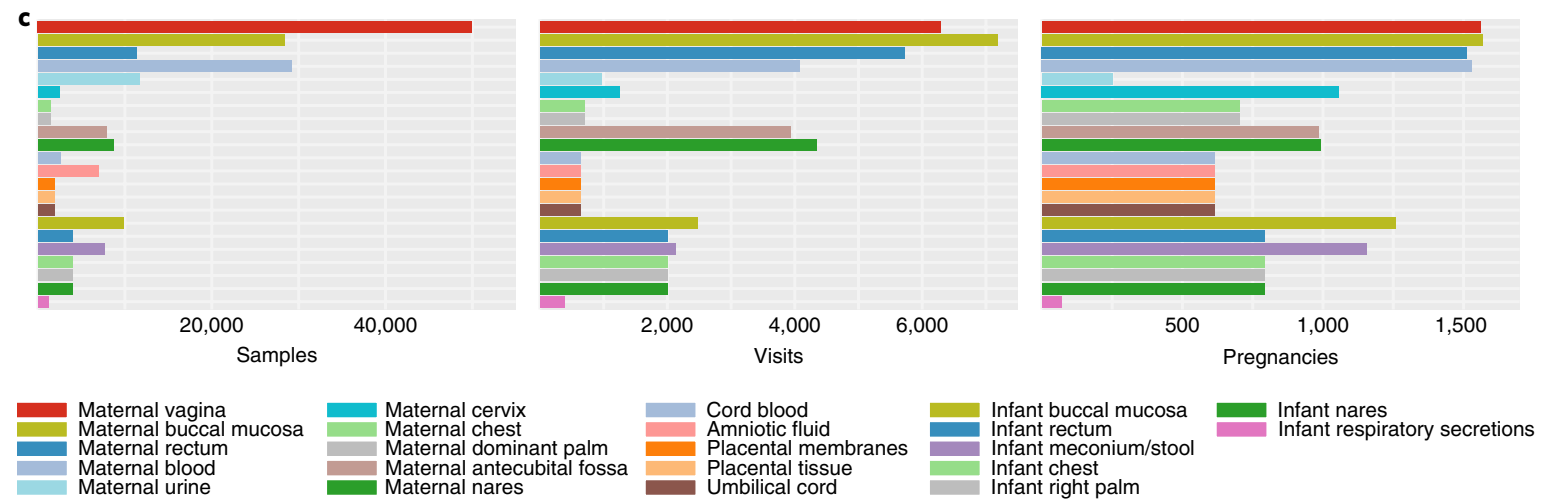

Fig. 1 | MOMS-PI resources. a, An overview of the study designs for the MOMS-PI PTB study (45 spontaneous preterm (sPTB) cases and 90 term controls) and the MOMS-PI TB study (90 women who delivered at term or early term and their neonates). Both cohorts were selected from the phase 1 RAMS Registry cohort $(n=627)$. b, Omics data were generated from samples from the MOMS-PI PTB and MOMS-PI TB studies and 384 additional pregnancies from the overall MOMS-PI cohort. Samples from the 12 women who were selected for both the MOMS-PI PTB study and the MOMS-PI TB study are depicted under both studies. Omics data types include $16 \mathrm{~S}$ rRNA amplicon sequencing, metagenomic sequencing (MGS), metatranscriptomic sequencing (MTS), host cytokine assays and lipidomics. c, A total of 206,437 samples were collected at more than 7,000 visits from 1,572 pregnancies in the MOMS-PI study, and are archived in the RAMS Registry.

of 0.723 for samples not used during training. This model, based on microbiome composition data, had $5-7 \%$ greater sensitivity and specificity than a model constructed using only clinical variables with a slight reduction in the AUROC curve (that is, 0.723 versus 0.764). A network analysis of these four taxa (Fig. 2c) shows them to be positively correlated with taxa associated with vaginal dysbiosis. 
Table 1 | Description of cohort studied in this project

\begin{tabular}{|c|c|c|}
\hline & $\begin{array}{l}\text { Preterm delivery } \\
<37 \text { weeks } \\
(n=45)\end{array}$ & $\begin{array}{l}\text { Term delivery } \\
\geq 39 \text { weeks } \\
(n=90)\end{array}$ \\
\hline Mean age (years) & $26(5.68)$ & $25.9(5.43)$ \\
\hline \multicolumn{3}{|l|}{ Ancestry/ethnicity (no. (\%)) } \\
\hline African & $35(77.8)$ & $71(78.9)$ \\
\hline European & $6(13.3)$ & $13(14.4)$ \\
\hline Hispanic & $3(6.7)$ & $5(5.6)$ \\
\hline Native American & $1(2.2)$ & $1(1.1)$ \\
\hline \multicolumn{3}{|l|}{ Household income (no. (\%)) ${ }^{b}$} \\
\hline$<$ US $\$ 20,000$ & $29(72.5)$ & $66(77.7)$ \\
\hline US $\$ 20,000-59,999$ & $9(22.5)$ & $15(17.6)$ \\
\hline US\$60,000+ & $2(5.0)$ & $4(4.7)$ \\
\hline Vaginal delivery (no. (\%)) & $38(84.4)$ & $74(82.2)$ \\
\hline Previous preterm (no. (\%)) & $14(31.1)$ & $9(10.0)$ \\
\hline $\begin{array}{l}\text { Preterm premature rupture of the } \\
\text { membranes (no. (\%)) }\end{array}$ & $26(57.8)$ & $0(0)$ \\
\hline
\end{tabular}

a Standard deviation listed in parentheses. ${ }^{b}$ Missing values $n=5$ (PTB), $n=5$ (TB).

We further examined longitudinal trends of key taxa using a generalized additive mixed effect model (GAMM) incorporating body mass index (BMI), vaginal $\mathrm{pH}$, ethnicity and preterm status (Fig. 3a, and see Supplementary Fig. 1a). Many taxa identified as associated with PTB in the $16 \mathrm{~S}$ rRNA cross-sectional analyses, including $S$. amnii $(P=0.0015)$, Prevotella cluster $2(P=0.0031)$, BVAB1 $(P=0.0037)$ and $P$ amnii $(P=0.0031)$, were also associated with PTB in this longitudinal analysis. Women who delivered preterm experienced large decreases during pregnancy in $S$. amnii $(P=0.0163)$, BVAB1 $(P=0.0002), P$. amnii $(P=0.0004)$, Gardnerella vaginalis $(P=0.0074)$, TM7-H1 $(P=0.0005)$ and Atopobium vaginae $(P=0.0090)$. Prevotella cluster 2 also showed a decreased prevalence later in pregnancy, but the decrease was not statistically significant. In contrast, $L$. crispatus increased in prevalence $(P=0.0320)$ over the course of the pregnancy in women who delivered preterm. Women who delivered at term exhibited significant decreases in prevalence of $A$. vaginae $(P<0.0001)$ and $G$. vaginalis $(P=0.0012)$, and an increase in L. iners $(P=0.0273)$.

A stratification of the longitudinal GAMM analysis by ancestry (Fig. 3b, and see Supplementary Fig. 1b) showed that, over the duration of pregnancy, women of African ancestry who delivered preterm experienced significant decreases in the prevalence of $A$. vaginae $(P=0.0011)$, BVAB1 $(P=0.0003), G$. vaginalis $(P=0.0002)$, $P$. amnii $(P=0.0013), S$. amnii $(P=0.0219)$ and TM7H1 $(P=0.0014)$. Women of African ancestry $(\mathrm{AA})$ who delivered at term exhibited fewer changes in the modeled taxa throughout pregnancy, although decreases in $A$. vaginae $(P=0.0001)$ and $G$. vaginalis $(P=0.0003)$ and an increase in $L$. iners $(P=0.0404)$ were observed. Women of European ancestry (EA) generally exhibited stable microbiome profiles during pregnancy, although an increase in prevalence of $G$. vaginalis $(P=0.0401)$ was noted for women who delivered preterm. G. vaginalis has been previously reported as a microbial signature for PTB in cohorts of women of predominantly European ancestry ${ }^{25}$. Overall, our observations are consistent with previous reports of dynamic changes in the vaginal microbiome in pregnancy ${ }^{18,48,49}$ and results from the MOMS-PI TB study, which show that the dynamics of vaginal microbiome differ by ancestry, with women of African ancestry exhibiting a more pronounced decrease in microbial diversity throughout the course of a term pregnancy ${ }^{42}$.
A totalof496longitudinalvaginal samples from participantsin the MOMS-PI PTB study (see Fig. 1b) were subjected to metagenomic sequencing (MGS) and a subset of 243 samples was subjected to metatranscriptomic sequencing (MTS) (see Extended Data Fig. 4). At the pathway level, the functional and metabolic potentials of the microbial communities were largely conserved, with the exception of $L$. crispatus-dominated samples, which exhibited a much higher proportional metabolic potential and transcriptional activity of the UDP- $N$-acetyl-D-glucosamine (UDP-GlcNAc) biosynthesis pathway (see Extended Data Fig. 5). UDP-GlcNAc is a precursor for peptidoglycan, one of the best-described, microbe-associated molecular patterns involved in the modulation of host cytokine production via toll-like receptor signaling ${ }^{50}$. Additional studies are required to determine whether combinations of microbeassociated molecular patterns produced by the vaginal microbiota modulate host cytokine levels and impact urogenital health. In contrast, the proportional transcriptional activities of genes classified to the pathway of pyruvate fermentation to acetate and lactate II, and the non-oxidative branch of the pentose phosphate pathway, were lower in L. crispatus-dominated samples (see Extended Data Fig. 5). This finding is consistent with reports of reduced levels of lactic acid and increased concentrations of short-chain fatty acids in vaginal samples of women with bacterial vaginosis ${ }^{51}$. Short-chain fatty acids have been suggested to reduce antimicrobial activity and promote proinflammatory cytokines in the vaginal environment.

Metagenomic assembly of reference genomes of bacterial taxa associated with PTB. MGS data generated with Pacific BioSciences and Illumina sequencing technologies were used to generate the first genomes of TM7-H1 (CP026537) and BVAB1 (PQVO000000), respectively. BVAB1, with a genome of $\sim 1.45$ megabases $(\mathrm{Mb})$, is classified to the Family Lachnospiraceae of the Order Clostridiales, and is not closely related to any other known bacterium (see Supplementary Table 6). TM7-H1, with a genome of $\sim 0.72 \mathrm{Mb}$, falls into the Phylum Candidatus Saccharibacteria and exhibits only $\sim 66 \%$ nucleotide identity with the recently described oral TM7x isolate (NZ_CP007496) ${ }^{52}$. TM7$\mathrm{H} 1$ encodes a putative $\alpha$-amylase and is predicted to be able to utilize glycogen as a carbon source (see Supplementary Data 1). Similar to TM7 $\mathrm{x}^{52}$, TM7-H1 lacks de novo biosynthetic capabilities for essential amino acids (see Supplementary Table 7), and likely depends on other organisms in the vaginal environment for survival. However, although TM7x is an obligate parasitic epibiont, it remains unknown whether TM7-H1 similarly lives on the surface of another bacterial species in the vaginal environment. We identified 243 and 421 metabolic reactions, respectively, in TM7-H1 and BVAB1 (see Supplementary Tables 7 and 8, and Supplementary Data 2). Both organisms are predicted to have the ability to produce pyruvate, acetate, L-lactate and propionate. BVAB1 encodes additional pathways for production of acetaldehyde, D-lactate, formate and acetyl-CoA. Neither is predicted to have a functional tricarboxylate cycle, and TM7-H1 completely lacks genes related to butyrate metabolism. As described above, production of shortchain fatty acids has been linked to a proinflammatory state ${ }^{51}$, with possible implications for disease.

Bacterial taxa associated with PTB in metagenomic and metatranscriptomic data. On average, approximately $95 \%$ of MGS reads and $30 \%$ of MTS reads were identified as human (see Extended Data Fig. 4). Most non-human MGS and MTS reads mapped to our customized vaginal bacterial database, with only a small fraction remaining unmapped (that is, average of $0.45 \%$ fullterm metagenomics, $0.41 \%$ preterm metagenomics, $1.67 \%$ full-term metatranscriptomics and $2.46 \%$ preterm metatranscriptomics) (see Supplementary Data 3 ). We compared the relative proportional 


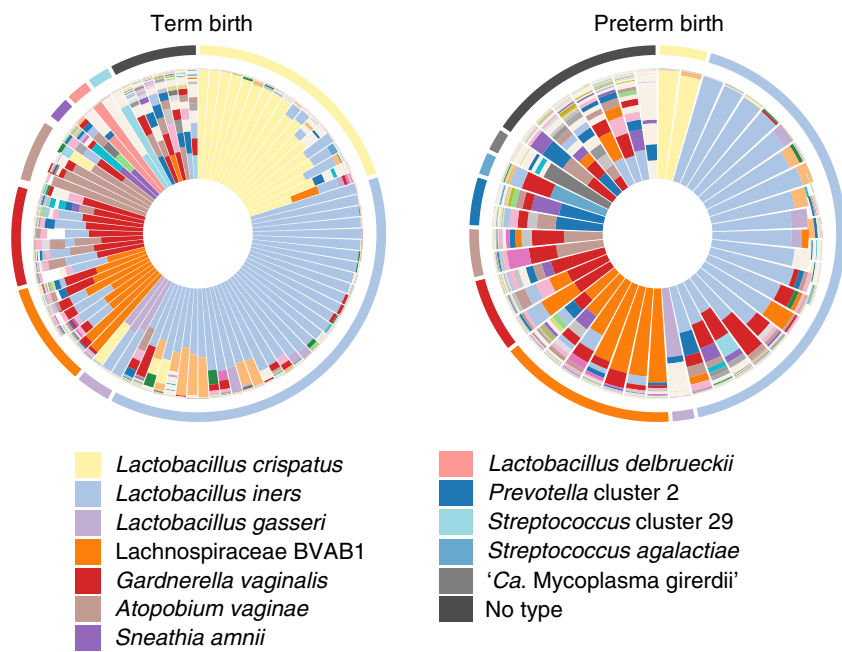

c

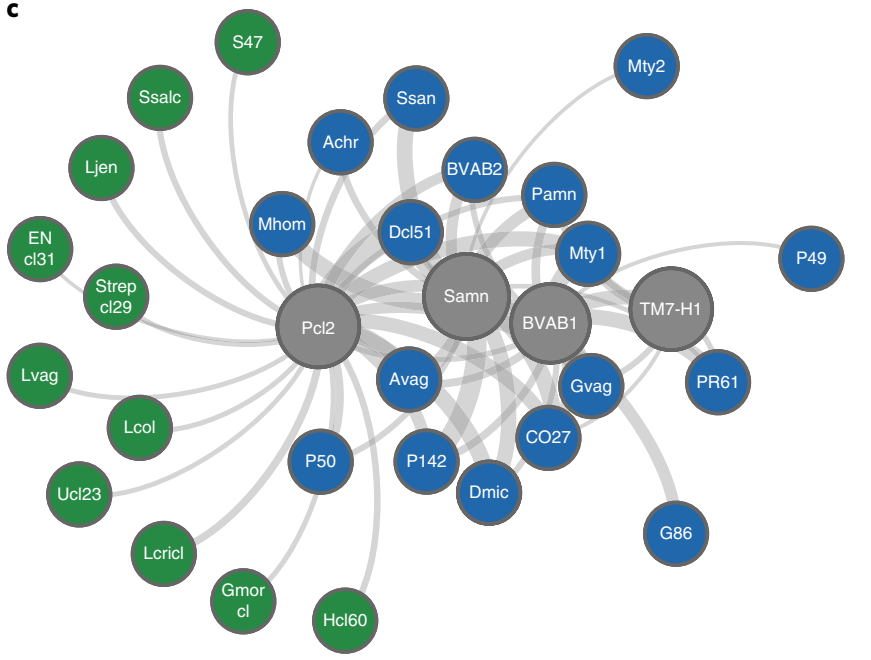

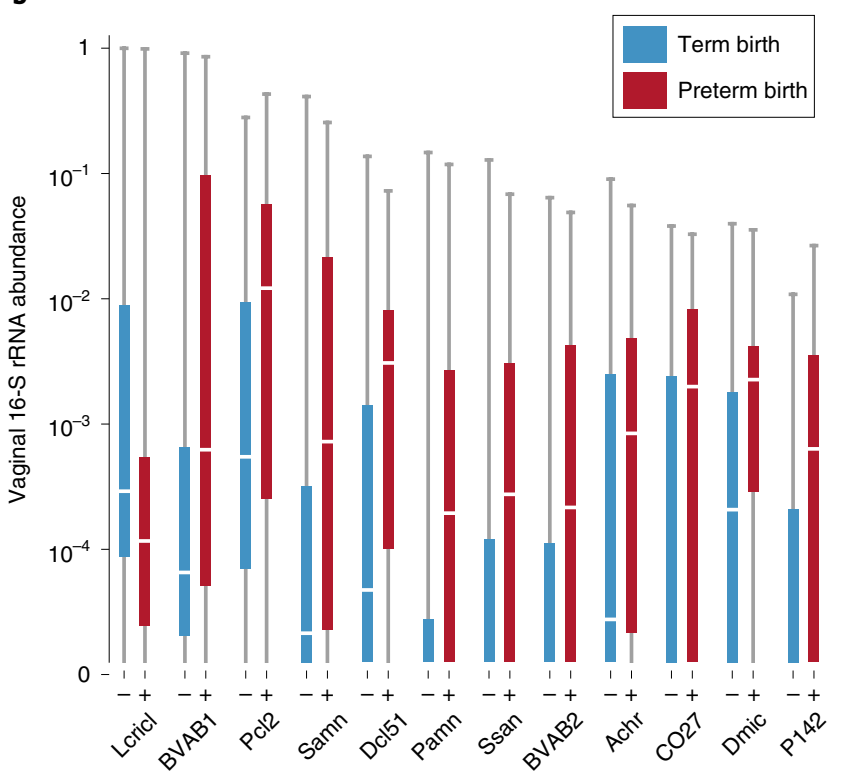

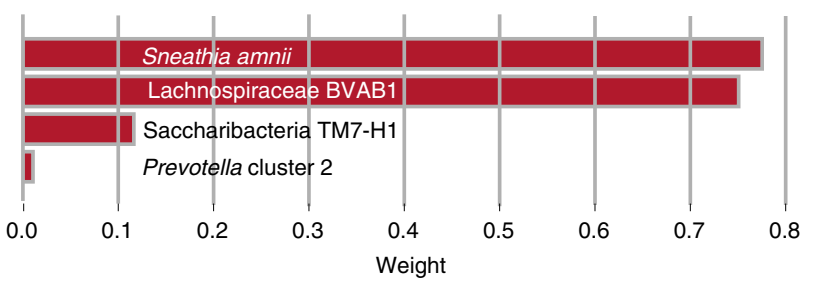

Fig. 2 | Bacterial taxa associated with spontaneous PTB. a, Vagitypes of 90 women who delivered at term ( $\geq 39$ weeks of gestation), and 45 women who delivered prematurely ( $<37$ weeks of gestation) showing 13 community states, or vagitypes. $\mathbf{b}$, Abundance of taxa significantly different in PTB $(n=45)$ and TB $(n=90)$ cohorts. These taxa have $P<0.05$ for the Mann-Whitney $U$-test (two-sided) for difference in proportional abundance between the cohorts, corrected using the Benjamini-Hochberg procedure with an FDR of 5\%. TB is indicated in blue as (-) and PTB in red as (+). Boxes show the median and interquartile range; whiskers extend from minimum to maximum values within each cohort. c, Network analysis of four taxa highly associated with PTBs. Negative correlations are shown in green, positive correlations in blue and predictive taxa in gray. Edge weights represent the strength of correlation. See Supplementary Table 3 for abbreviations. d, Predictive linear model for PTBs that produces a score based on weighted log(abundances) of four taxa in vaginal $16 \mathrm{~S}$ rRNA profiles in the 6- to 24-week gestational age range. Taxa abbreviations: Lcricl, L. crispatus cluster; BVAB1, Lachnospiraceae BVAB1; Pcl2, Prevotella cluster 2; Samn, S. amnii; Dcl51, Dialister cluster 51; Pamn, P. amnii; BVAB2, Clostridiales BVAB2; CO27, Coriobacteriaceae OTU27; Dmic, Dialister micraerophilus; P142, Parvimonas OTU142.

abundance of taxa from the 16S rRNA assay with the relative proportional abundance of metagenomic and metatranscriptomic data that mapped to non-ribosomal genes across the 56 taxa in our database. Although proportional differences were observed across detection methods, there was concordance in detection of taxa using 16S rRNA profiles, MGS and MTS (see Extended Data Fig. 6).

Paired MGS and MTS data were available for 41 women who delivered preterm and for 81 term controls. Thus, we analyzed a single time point per participant, with a mean gestational age of sampling at 25 weeks for the preterm cohort and 26 weeks for the full-term cohort; we also used a global scaling approach to normalize to all genes in the 56 taxa in our database. In the preterm samples, we observed higher transcript levels of genes from all of the taxa identified as candidate markers of PTB that were analyzed, with inclusion of samples collected even later in pregnancy than those used for $16 \mathrm{~S}$ rRNA analyses (Fig. 4). Conversely, we observed higher transcript levels of $L$. crispatus in the term samples. L. jensenii, L. gasseri, L. iners and G. vaginalis had relatively few genes that showed very different transcript levels between the term and preterm cohorts (Fig. 4).

Using the same approach with MGS data, we observed similar trends, but fewer genes were identified as statistically significant $\left(P_{\text {adj }}<0.05\right)$ overall (see Extended Data Fig. 7a and Supplementary Tables 9 and 10). Interestingly, $12.55 \%$ of the G. vaginalis genes analyzed using MGS data were significantly higher in the term cohort, whereas only one gene (that is, $0.02 \%$ of genes analyzed) that was identified as a hypothetical protein was higher $\left(P_{\text {adj }}<0.05\right)$ in the preterm cohort. We found the overall relative transcriptional rate of G. vaginalis was higher in preterm samples compared with term samples, using a calculated ratio of the proportion of reads mapped to genes in $G$. vaginalis reference genomes by MTS to reads mapped by MGS (Wilcoxon's, $P<0.05$; see Extended Data 

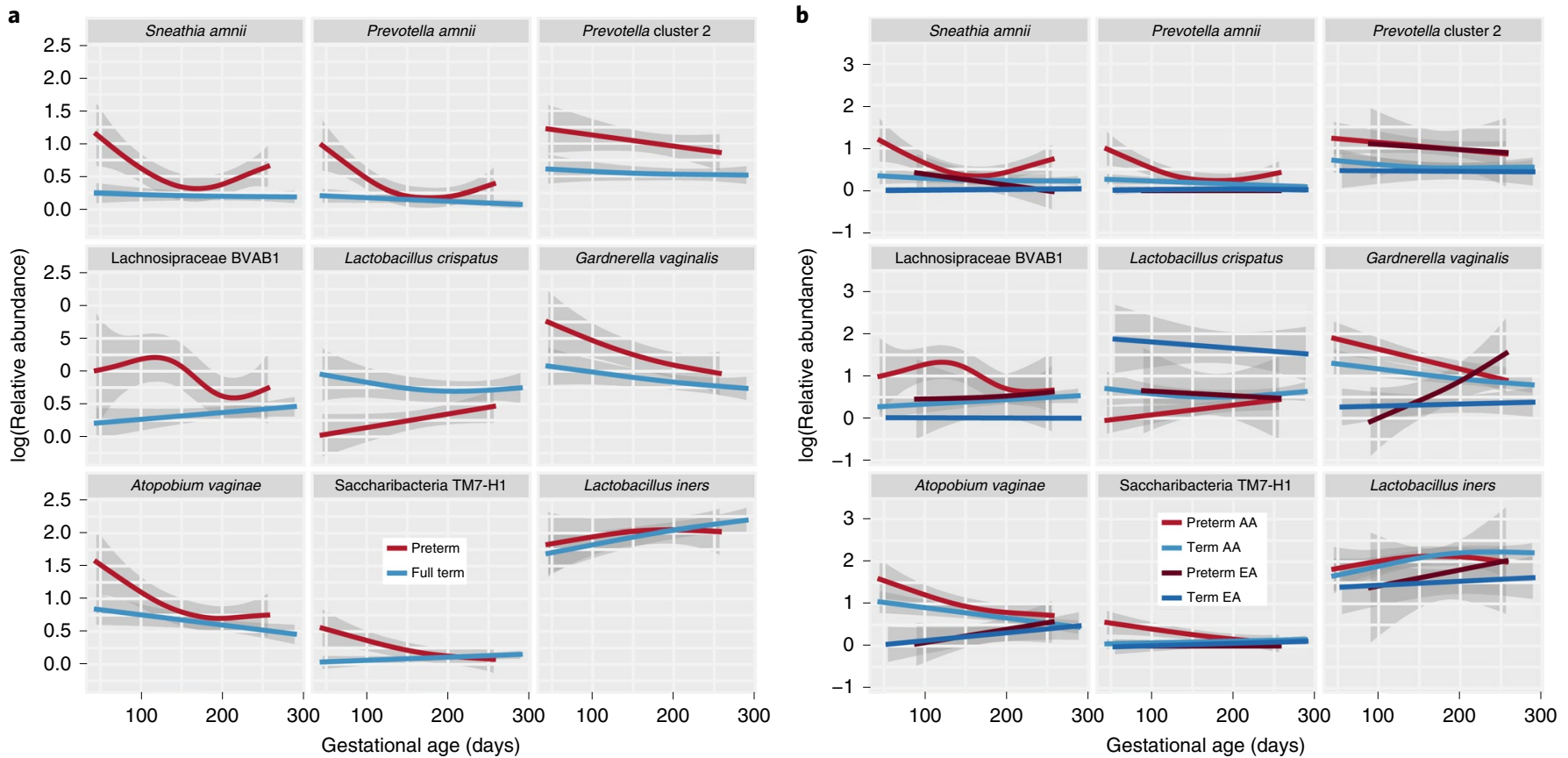

Fig. 3 | Longitudinal GAMM of vaginal microbiome composition during pregnancy. The model incorporates BMI, vaginal pH, pregnancy outcome (PTB, TB), a smoother for gestational age and a random subject effect to longitudinally model log-transformed relative abundances of vaginally relevant taxa (see Methods). Each figure plots log-transformed abundances of taxa throughout pregnancy. a, Plots comparing the PTB case ( $n=41)$ and TB cohorts $(n=90)$. b, Comparison of the results from preterm and full-term women of African and European ancestry (that is, EA PTB, $n=7 ;$ AA PTB, $n=31 ;$ EA TB, $n=13 ; \mathrm{AA} T \mathrm{~TB}, n=73$ ). Confidence intervals (98\%) are shown.
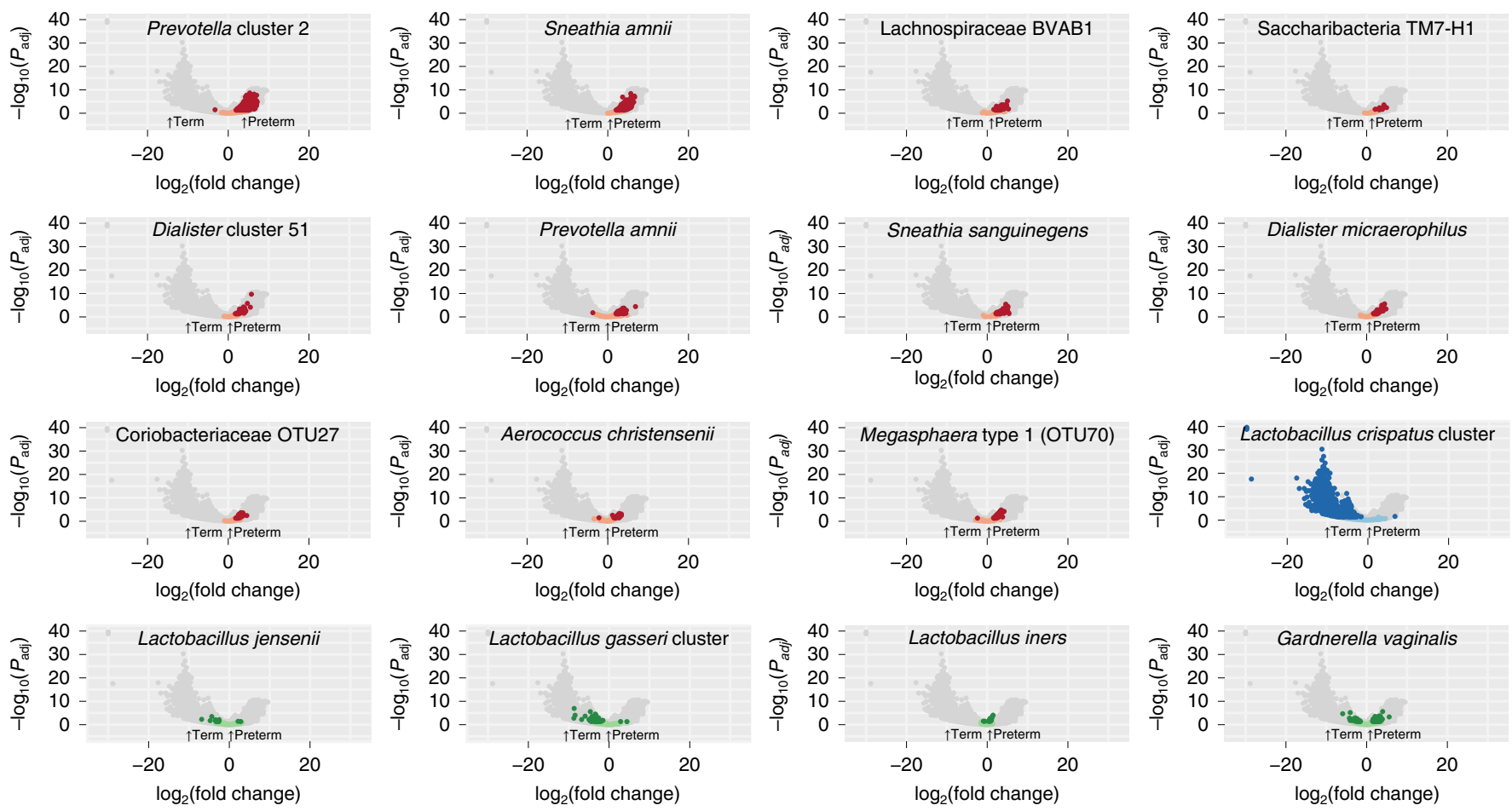

Fig. 4 | Volcano plot depicting transcripts that differ greatly between term and preterm cohorts in metatranscriptomics data for taxa of interest.

Analysis was performed by mapping reads from PTB $(n=41)$ and TB $(n=81)$ samples to a custom database of genomes representing 56 taxa.

Comparative analysis was performed with DESeq2 using a global scaling approach. Genes in candidate PTB taxa identified in $16 \mathrm{~S} r \mathrm{RNA}$ analyses that differ significantly at $P_{\text {adj }}<0.05$ with a two-sided Wald test, as implemented in DESeq2 with a Benjamini-Hochberg FDR correction, are shown in red and those that were not statistically significant are shown in pink. Genes are also shown for the L. crispatus cluster (associated with TB in the 165 rRNA analyses), with genes that differ significantly $\left(P_{\text {adj }}<0.05\right)$ in transcript levels shown in dark blue and those that do not in light blue. Four other common and abundant taxa (L. jensenii, L. gasseri cluster, L. iners and G. vaginalis) are shown, with dark green dots denoting genes that differ significantly $\left(P_{\text {adj }}<0.05\right)$ between cohorts and light green dots denoting those that were not statistically significant. 
Fig. 7b,c). Previous studies have suggested that PTB risk differs with carriage of different G. vaginalis clades $^{25}$. We extend this finding by showing data suggesting that PTB risk also differs with the transcriptional activity of G. vaginalis. Further investigations will be required to determine the underlying mechanisms that impact replication and transcription of different strains of $G$. vaginalis during pregnancy, and how these mechanisms affect women's reproductive health and pregnancy.

Genes encoding proteins involved in bacterial secretion systems play an important role in pathogenicity ${ }^{53}$. Thus, we examined all genes predicted to encode proteins involved in bacterial secretion in the database. We observed that $81 \%(71 / 91)$ of the predicted genes encoding secreted proteins that were far more transcriptionally abundant in the preterm cohort were from taxa identified as associated with PTB in our 16S rRNA analyses (see Extended Data Fig. 8a, Supplementary Tables 11 and 12, and Supplementary Data 4). The detected differences between the PTB and TB samples likely reflect the elevated proportional abundance of these taxa in PTB samples. Further studies are needed to determine whether these genes may contribute to mechanisms by which components of the vaginal microbiome may mediate or cause pathology or PTBs.

Host cytokine expression in PTB. Of the nine cytokine levels examined in the present study (interleukin (IL)-1 $\beta$, IL-6, IL-8, eotaxin, tumor necrosis factor (TNF)- $\alpha$, IL-17A, macrophage inflammatory protein (MIP)- $1 \beta$, interferon- $\gamma$-induced protein (IP)$10 /$ chemokine ligand (CXCL) 10, RANTES (regulated on activation, normal $\mathrm{T}$ cell expressed and secreted)), four (eotaxin, IL-1 $\beta$, IL-6 and MIP-1 $\beta$ ) were greatly increased in PTB relative to TB samples (false discovery rate (FDR)-adjusted $P<0.05$ for each), consistent with previous reports of elevated IL-1, IL-6, MIP-1, IP10/CXCL10 and other proinflammatory cytokines associated with PTB in blood, amniotic fluid or cervical-vaginal lavage samples ${ }^{54}$. For further examination of the role of cytokines in the progression of pregnancy to PTB, we performed an integrative sparse canonical correlation analysis (sCCA) to assess the association of specific bacterial taxa with the abundance levels of nine key cytokines. For each participant, the sample corresponding to the earliest gestational age per trimester was characterized. In women who delivered at term (Fig. 5 a), we observed a strong negative correlation between $L$. crispatus and several taxa associated with dysbiosis and PTB (for example, $G$. vaginalis, Prevotella cluster 2, S. amnii and, to a lesser extent, TM7$\mathrm{H1}$ ), as well as with the analyzed cytokines. The analyzed cytokines, which are largely proinflammatory, were loosely correlated both with each other and with taxa associated with dysbiosis and PTB. Notably, IP-10/CXCL10, which functions to induce chemotaxis of immune cells and promotes apoptosis, cell growth and angiostasis, and is generally considered to be proinflammatory ${ }^{55}$, was positively correlated with $L$. iners. This association was previously reported in the reproductive tracts of non-pregnant women from Kenya ${ }^{56}$. In contrast, in women who went on to experience PTB (Fig. 5b), the proinflammatory cytokines and dysbiotic taxa (for example, A. vaginae, G. vaginalis and Megasphaera type 1) formed a tighter cluster, indicating a stronger positive correlation, but IP-10/CXCL10 did not correlate with $L$. iners. Furthermore, BVAB1 was negatively correlated with IP-10/CXCL10 in these samples.

Cross-study comparisons of the vaginal microbiome and preterm birth. Several recent studies ${ }^{25,27,28,30}$ generally reported limited correlation between the composition of the vaginal microbiome and PTB in cohorts of African descent. We compared the distributions of distinct candidate taxa for PTB risk across four studies ${ }^{25,27,30}$ of the vaginal microbiome in cohorts of pregnant women with predominantly African ancestry, including the MOMS-PI PTB study, with a harmonized reanalysis of the raw $16 \mathrm{~S}$ rRNA sequencing reads (see Supplementary Tables 13 and 14, and Extended Data Figs. 9 and 10). There were non-trivial differences in every technical aspect of study design including sample collection, DNA extraction, PCR primers and conditions, sequencing platform, data quality and deposit which precluded an integrated analysis of these datasets (see Supplementary Table 13). Moreover, each of these studies varied markedly in cohort demographics, inclusion and exclusion criteria, and even the definition of PTB. PTB is understood to be a syndrome with many underlying causes ${ }^{9}$. Ascending infection of microbes from the vagina likely plays a causative role in some subtypes of PTB, but likely does not play a contributing role in all PTB. Thus, there were considerable differences (see Extended Supplementary Table 14) in the PTB case cohorts, as originally published in terms of the distribution of gestational age at delivery (see Extended Data Fig. 9), the percentage of women who had a non-medically indicated spontaneous PTB (37.5-100\%), preterm premature rupture of the membranes (12.5-57.8\%), treatment with progesterone (0-100\%) or a history of PTB (29.1-100\%). Our attempts to aggregate data from different studies highlight the opportunities for harmonization to obtain comparable data across studies.

We examined only the spontaneous preterm cases and we rematched controls 1:2 using the same approach from the present study to harmonize study designs across studies as far as possible for reanalysis. Sample sizes were small, in the range 5-18 spontaneous PTB cases, with controls matched 1:2. Although not statistically significant, likely due to sample size, cohort characteristics, and differences in experimental design and details as outlined above, we found partial support for candidate taxa identified in the present study (see Extended Data Fig. 10a-d). For example, the present study confirms an association between Sneathia sanguinegens and PTB, which was reported as significant $(P<0.05)$ before adjustment for multiple testing by Romero et al. ${ }^{30}$. Although there were only five spontaneous PTB cases reanalyzed in the Stout et al. cohort $^{27}$, and only ten in the reanalyzed Callahan et al..$^{25}$ cohort, we observed high concordance in the directionality of differences in abundance levels of preterm and term groups between these cohorts and the present study. We were also able to confirm that BVAB1, Megasphaera phylotype 1 and Sneathia species were elevated in a preterm cohort, which Nelson et al. ${ }^{26}$ previously reported as related to an increased risk for PTBs among women reporting a prior preterm delivery. The Nelson et al. ${ }^{26}$ study used quantitative PCR rather than 16S rRNA profiling, and the study was thus not included in the harmonized reanalysis. We also confirmed that $L$. iners and G. vaginalis, which were identified as vaginal microbial signatures associated with PTB in low-risk cohorts ${ }^{25}$, did not generalize to cohorts of African ancestry.

\section{Discussion}

From a subset of 597 of the 1,572 pregnancies longitudinally sampled for the MOMS-PI study, we have generated omics data from more than 12,000 samples in one of the largest and most comprehensive multi-omic studies published to date. In addition, our analyses of longitudinal omics data from vaginal samples from 45 women who delivered preterm and 90 controls showed a signature of PTB in a cohort of women of predominantly African ancestry, including several taxa that have previously been implicated in adverse outcomes of pregnancy, including premature delivery ${ }^{22,26,57-59}$, in addition to taxa that have not been previously linked to adverse pregnancy outcomes. Women of African ancestry have a greatly increased risk of PTB compared with women of European ancestry ${ }^{60}$. Previous studies $^{16,21}$ have shown that carriage of $L$. crispatus, which is negatively associated with PTB (see Fig. 2 and refs. ${ }^{22-25}$ ), is more prevalent in women of European ancestry, and BVAB1, which is positively associated with PTBs, is more common in women of African ancestry. Thus, our findings are consistent with a proposed framework in which there is a spectrum of vaginal microbiome states linked to risk for PTB, and that these states vary across populations. 

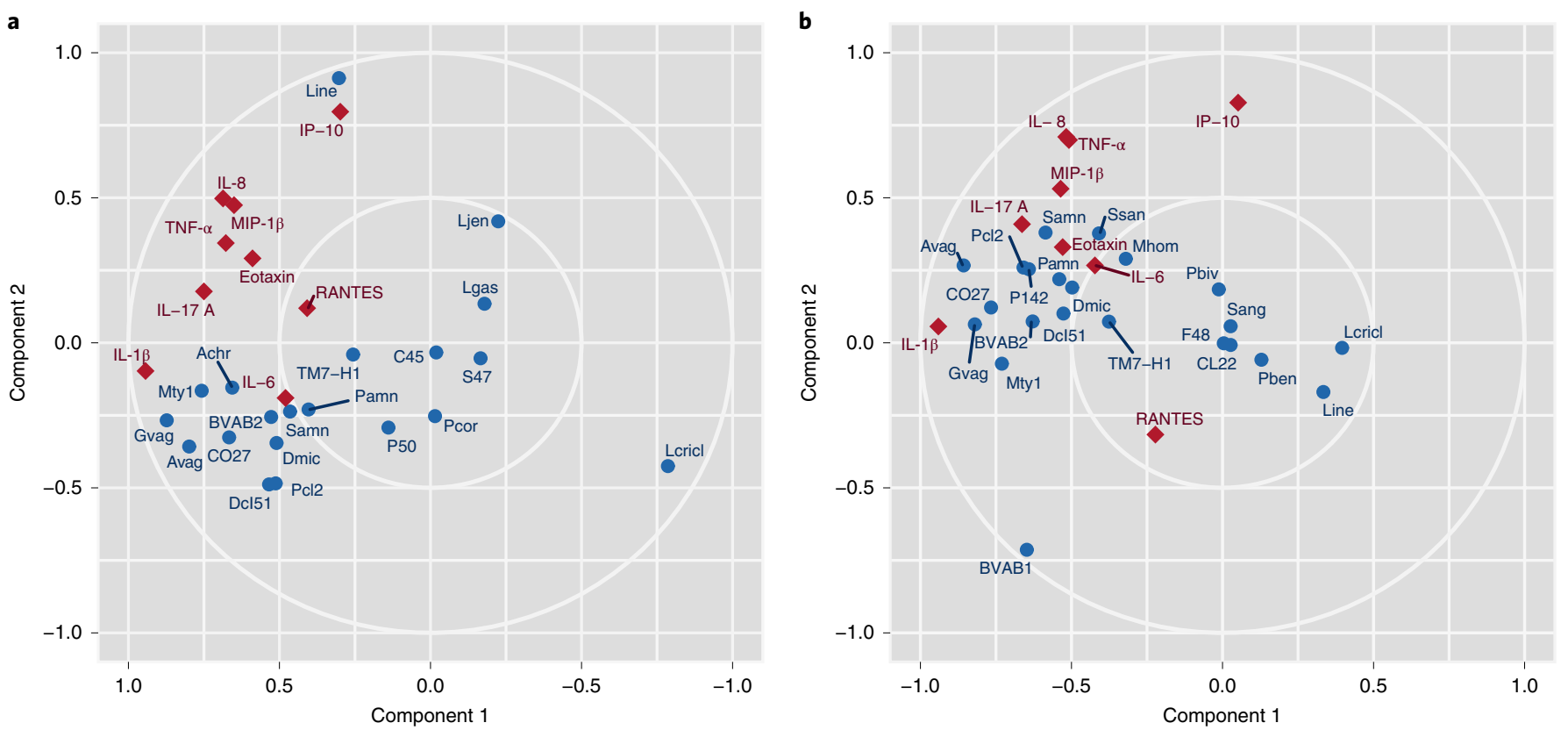

Fig. 5 | Sparse canonical correlation analysis. a,b, Cytokine abundance in vaginal samples from women who experienced TB $(n=90)(\mathbf{a})$ or PTB $(n=41)$ (b) were subjected to an integrative SCCA using log-transformed cytokine levels and log-transformed taxonomic profiling data (see Methods). Blue circles represent bacterial taxa and red diamonds represent cytokines. Note that the component 1 axis for the TB sCCA (left) has been reversed for effective visual comparison with PTB sCCA. See Supplementary Table 3 for abbreviations. Note that, in sCCA analysis, factors (cytokines or microbial taxa) that are clustered tightly are highly correlated, and factors that are distant from each other are inversely correlated.

Our longitudinal modeling showed taxa associated with PTB tended to decrease in abundance in the vaginal environment throughout pregnancy, particularly in women of African ancestry. This finding is consistent with previous observations that pregnancy is associated with reduced carriage of bacterial vaginosisassociated organisms ${ }^{21,27,57}$. Considering that an adverse pregnancy outcome may be caused by ascension of pathogenic microbes, this trend suggests that the microbiome composition early in pregnancy may be most useful in the prediction of adverse outcomes. In a complementary analysis of an ethnically diverse cohort of women who delivered at term or early term in the MOMS-PI TB study, we show differences in the longitudinal dynamics of the microbiome in women of African ancestry compared with women of European ancestry $^{42}$. In the present study, we developed a proof-of-concept model that suggests the presence of BVAB1, Prevotella cluster 2, S. amnii and TM7-H1 early in pregnancy may be useful for prediction of risk for PTB, particularly in high-risk populations. It is possible that BVAB1, Prevotella cluster 2, S. amnii and TM7-H1, and other taxa may have roles in the causation of PTB. As BVAB1 and TM7-H1 had not been cultivated or genetically characterized, we assembled their genomes from MGS data to search for clues to their pathogenic potential. We previously characterized the genome of $S$. amnii, identified potential cytotoxin genes and showed that cultured bacteria kill eukaryotic cells in vitro ${ }^{61}$. Although culture is not available for BVAB1 or TM7-H1, genomic factors identified in their genomes can now be genetically amplified and recombined into heterologous reporter systems and tested for pathogenic activity. Our MTS and MGS analyses supported the microbial signatures identified using $16 \mathrm{~S}$ rRNA profiles.

Analysis of vaginal cytokine data from the MOMS-PI PTB study is consistent with previous findings showing that bacterial taxa generally associated with dysbiosis are highly correlated with expression of proinflammatory cytokines, which may play a role in the induction of labor. Labor is associated with proinflammatory cytokine expression, and premature labor can be induced by host inflammatory responses. We observed that vaginal IP-10/CXCL10 levels were inversely correlated with BVAB1 in PTB, inversely correlated with $L$. crispatus in TB and positively correlated with $L$. iners in TB, suggesting complex host-microbiome interactions in pregnancy.

Our findings contribute to an understanding of how microbial markers for PTB vary across populations. Vaginal microbiome composition as a whole and carriage rates of specific microbial taxa vary dramatically across populations, and thus it is not unexpected that the importance of relevant markers differs accordingly. Further studies are needed to determine whether the signatures of PTB reported in the present study replicate in other cohorts of women of African ancestry, to examine whether the observed differences in vaginal microbiome composition between women of different ancestries has a direct causal link to the ethnic and racial disparities in PTB rates, and to establish whether population-specific microbial markers can be ultimately integrated into a generalizable spectrum of vaginal microbiome states linked to the risk for PTB. Taken together, our data suggest that, coupled with other clinical and possibly genetic factors, microbiome-associated taxonomic, metabolic and immunologic biomarkers may be useful in defining the risk of $\mathrm{PTB}$, and that this risk might be assessed early in pregnancy.

\section{Online content}

Any methods, additional references, Nature Research reporting summaries, source data, statements of code and data availability and associated accession codes are available at https://doi.org/10.1038/ s41591-019-0450-2.

Received: 19 December 2018; Accepted: 9 April 2019;

Published online: 29 May 2019

\section{References}

1. Blencowe, H. et al. National, regional, and worldwide estimates of preterm birth rates in the year 2010 with time trends since 1990 for selected countries: a systematic analysis and implications. Lancet 379, 2162-2172 (2012).

2. Liu, L. et al. Global, regional, and national causes of under-5 mortality in 2000-15: an updated systematic analysis with implications for the Sustainable Development Goals. Lancet 388, 3027-3035 (2016). 
3. Marret, S. et al. Neonatal and 5-year outcomes after birth at 30-34 weeks of gestation. Obstet. Gynecol. 110, 72-80 (2007).

4. Wolke, D., Eryigit-Madzwamuse, S. \& Gutbrod, T. Very preterm/very low birthweight infants' attachment: infant and maternal characteristics. Arch. Dis. Child. Fetal Neonat. Ed. 99, F70-F75 (2014).

5. Behrman, R. E. \& Butler, A. S. (eds), for Institute of Medicine Committee on Understanding Premature Birth and Assuring Healthy Outcomes. Societal Costs of Preterm Birth (National Academies Press, 2007).

6. Strauss, J. F. et al. Spontaneous preterm birth: advances toward the discovery of genetic predisposition. Am. J. Obstet. Gynecol. 218, 294-314.e2 (2018).

7. Goldenberg, R. L., Culhane, J. F., Iams, J. D. \& Romero, R. Epidemiology and causes of preterm birth. Lancet 371, 75-84 (2008).

8. Donders, G. G. et al. Predictive value for preterm birth of abnormal vaginal flora, bacterial vaginosis and aerobic vaginitis during the first trimester of pregnancy. Br. J. Obstet. Gynaecol. 116, 1315-1324 (2009).

9. Romero, R., Dey, S. K. \& Fisher, S. J. Preterm labor: one syndrome, many causes. Science 345, 760-765 (2014).

10. Lamont, R. F. Infection in the prediction and antibiotics in the prevention of spontaneous preterm labour and preterm birth. Br. J. Obstet. Gynaecol. 110 (Suppl 20), 71-75 (2003).

11. Lockwood, C. J. Predicting premature delivery-no easy task. N. Engl. J. Med. 346, 282-284 (2002).

12. Fredricks, D. N., Fiedler, T. L., Thomas, K. K., Oakley, B. B. \& Marrazzo, J. M. Targeted PCR for detection of vaginal bacteria associated with bacterial vaginosis. J. Clin. Microbiol. 45, 3270-3276 (2007).

13. Sobel, J. D. Bacterial vaginosis. Annu. Rev. Med. 51, 349-356 (2000)

14. Bradshaw, C. S. \& Sobel, J. D. Current treatment of bacterial vaginosis: limitations and need for innovation. J. Infect. Dis. 214, S14-S20 (2016).

15. Chavoustie, S. E. et al. Experts explore the state of bacterial vaginosis and the unmet needs facing women and providers. Int. J. Gynaecol. Obstet. 137, 107-109 (2017).

16. Ravel, J. et al. Vaginal microbiome of reproductive-age women. Proc. Natl Acad. Sci. USA 108 (Suppl 1), 4680-4687 (2011).

17. Ma, B., Forney, L. J. \& Ravel, J. Vaginal microbiome: rethinking health and disease. Annu. Rev. Microbiol. 66, 371-389 (2012).

18. MacIntyre, D. A. et al. The vaginal microbiome during pregnancy and the postpartum period in a European population. Sci. Rep. 5, 8988 (2015).

19. Martin, D. H. \& Marrazzo, J. M. The vaginal microbiome: current understanding and future directions. J. Infect. Dis. 214 (Suppl 1), S36-S41 (2016).

20. Zhou, X. et al. Differences in the composition of vaginal microbial communities found in healthy Caucasian and black women. ISME J. 1, 121-133 (2007).

21. Fettweis, J. M. et al. Differences in vaginal microbiome in African American women versus women of European ancestry. Microbiology 160, 2272-2282 (2014).

22. Brown, R. G. et al. Vaginal dysbiosis increases risk of preterm fetal membrane rupture, neonatal sepsis and is exacerbated by erythromycin. BMC Med. 16, 9 (2018).

23. Kindinger, L. M. et al. The interaction between vaginal microbiota, cervical length, and vaginal progesterone treatment for preterm birth risk. Microbiome 5, 6 (2017)

24. DiGiulio, D. B. et al. Temporal and spatial variation of the human microbiota during pregnancy. Proc. Natl Acad. Sci. USA 112, 11060-11065 (2015).

25. Callahan, B. J. et al. Replication and refinement of a vaginal microbial signature of preterm birth in two racially distinct cohorts of US women. Proc. Natl Acad. Sci. USA 114, 9966-9971 (2017).

26. Nelson, D. B. et al. Early pregnancy changes in bacterial vaginosis-associated bacteria and preterm delivery. Paediatr. Perinat. Epidemiol. 28, 88-96 (2014).

27. Stout, M. J. et al. Early pregnancy vaginal microbiome trends and preterm birth. Am. J. Obstet. Gynecol. 217, 356.e1-356.e18 (2017).

28. Nelson, D. B., Shin, H., Wu, J. \& Dominguez-Bello, M. G. The gestational vaginal microbiome and spontaneous preterm birth among nulliparous African American women. Am. J. Perinatol. 33, 887-893 (2016)

29. Han, Y. W., Shen, T., Chung, P., Buhimschi, I. A. \& Buhimschi, C. S. Uncultivated bacteria as etiologic agents of intra-amniotic inflammation leading to preterm birth. J. Clin. Microbiol. 47, 38-47 (2009).

30. Romero, R. et al. The vaginal microbiota of pregnant women who subsequently have spontaneous preterm labor and delivery and those with a normal delivery at term. Microbiome 2, 18 (2014).

31. Tabatabaei, N. et al. Vaginal microbiome in early pregnancy and subsequent risk of spontaneous preterm birth: a case-control study. Br. J. Obstet. Gynaecol. 126, 349-358 (2018).

32. Son, K.-A. et al. Prevalence of vaginal microorganisms among pregnant women according to trimester and association with preterm birth. Obstet. Gynecol. Sci. 61, 38-47 (2018).

33. Haque, M. M., Merchant, M., Kumar, P. N., Dutta, A. \& Mande, S. S. First-trimester vaginal microbiome diversity: a potential indicator of preterm delivery risk. Sci. Rep. 7, 16145 (2017).
34. Freitas, A. C. et al. The vaginal microbiome of pregnant women is less rich and diverse, with lower prevalence of Mollicutes, compared to non-pregnant women. Sci. Rep. 7, 9212 (2017).

35. Subramaniam, A. et al. Vaginal microbiota in pregnancy: evaluation based on vaginal flora, birth outcome, and race. Am. J. Perinatol. 33, 401-408 (2016).

36. Hyman, R. W. et al. Diversity of the vaginal microbiome correlates with preterm birth. Reprod. Sci. 21, 32-40 (2014).

37. Stafford, G. P. et al. Spontaneous preterm birth is associated with differential expression of vaginal metabolites by lactobacilli-dominated microflora. Front. Physiol. 8, 615 (2017).

38. Jefferson, K. K. et al. Relationship between vitamin D status and the vaginal microbiome during pregnancy. J. Perinatol. 39, 824-836 (2019).

39. Brown, R. G. et al. Prospective observational study of vaginal microbiota pre- and post-rescue cervical cerclage. Br. J. Obstet. Gynaecol. 126, 916-925 (2019).

40. Borgdorff, $\mathrm{H}$. et al. The association between ethnicity and vaginal microbiota composition in Amsterdam, the Netherlands. PloS ONE 12, e0181135 (2017).

41. The Integrative Human Microbiome Project. Dynamic analysis of microbiome-host omics profiles during periods of human health and disease. Cell Host Microbe 16, 276-289 (2014).

42. Serrano, M. G. et al. Nat. Med. https://doi.org/10.1038/s41591-019-0465-8 (2019)

43. Leal, M. et al. Burden of early-term birth on adverse infant outcomes: a population-based cohort study in Brazil. BMJ Open 7, e017789 (2017).

44. Murray, S. R. et al. Long term cognitive outcomes of early term (37-38 weeks) and late preterm (34-36 weeks) births: a systematic review. Wellcome Open Res. 2, 101 (2017).

45. Boyle, E. M. et al. Effects of gestational age at birth on health outcomes at 3 and 5 years of age: population based cohort study. BMJ 344, e896 (2012).

46. Brooks, J. P. et al. Changes in vaginal community state types reflect majo shifts in the microbiome. Microb. Ecol. Health Dis. 28, 1303265 (2017).

47. Fettweis, J. M. et al. Species-level classification of the vaginal microbiome. BMC Genom. 13 (Suppl 8), S17 (2012).

48. Romero, R. et al. The composition and stability of the vaginal microbiota of normal pregnant women is different from that of non-pregnant women. Microbiome 2, 4 (2014).

49. Walther-António, M. R. S. et al. Pregnancy's stronghold on the vaginal microbiome. PloS ONE 9, e98514 (2014).

50. Akira, S., Uematsu, S. \& Takeuchi, O. Pathogen recognition and innate immunity. Cell 124, 783-801 (2006).

51. Aldunate, M. et al. Antimicrobial and immune modulatory effects of lactic acid and short chain fatty acids produced by vaginal microbiota associated with eubiosis and bacterial vaginosis. Front. Physiol. 6, 164 (2015).

52. He, X. et al. Cultivation of a human-associated TM7 phylotype reveals a reduced genome and epibiotic parasitic lifestyle. Proc. Natl Acad. Sci. USA 112, 244-249 (2015)

53. Green, E. R. \& Mecsas, J. In Virulence Mechanisms of Bacterial Pathogens 5th edn (eds Kudva, I. et al.) 215-239 (2016).

54. Wei, S.-Q., Fraser, W. and Luo, Z.-C. Inflammatory cytokines and spontaneous preterm birth in asymptomatic women: a systematic review. Obstet. Gynecol. 116, 393-401 (2010).

55. Liu, M. et al. CXCL10/IP-10 in infectious diseases pathogenesis and potential therapeutic implications. Cytokine Growth Factor Rev. 22, 121-130 (2011).

56. Jespers, V. et al. A longitudinal analysis of the vaginal microbiota and vaginal immune mediators in women from sub-Saharan. Afr. Sci. Rep. 7, 11974 (2017).

57. Freitas, A. C., Bocking, A., Hill, J. E. \& Money, D. M. VOGUE Research Group. Increased richness and diversity of the vaginal microbiota and spontaneous preterm birth. Microbiome 6, 117 (2018).

58. Baldwin, E. A. et al. Persistent microbial dysbiosis in preterm premature rupture of membranes from onset until delivery. PeerJ 3, e1398 (2015).

59. Paramel Jayaprakash, T. et al. High diversity and variability in the vaginal microbiome in women following preterm premature rupture of membranes (PPROM): a prospective cohort study. PloS ONE 11, e0166794 (2016).

60. Blencowe, H. et al. Born too soon: the global epidemiology of 15 million preterm births. Reprod. Health 10, S2 (2013).

61. Harwich, M. D.Jr et al. Genomic sequence analysis and characterization of Sneathia amnii sp. nov. BMC Genom. 13 (Suppl 8), S4 (2012).

\section{Acknowledgements}

We gratefully acknowledge participants who contributed to the study and the teams of research coordinators, the sample processors and clinicians and nurses who assisted with sample collection. This study was funded by NIH grant nos. UH3AI083263 and U54HD080784 to G.A.B., K.K.J. and J.F.S. and was supported with funds from the Common Fund, the National Center for Complementary and Integrative Health, and the Office of Research on Women's Health. Other grants that provided partial support include a GAPPS BMGF PPB grant to G.A.B. and J.M.F., and NIH grants R21HD092965 to J.M.F. and E. Wickham, and 1R01HD092415 to G.A.B. T.A. was supported by NSF grant 1453658. N.R.J. was supported by grant no. R25GM090084 for the VCU Initiative for Maximizing Student Development program. Sequence analysis, with the exception of 
the TM7-H1 sequencing, was performed in the Nucleic Acids Research Facilities at VCU and analysis was performed with servers provided by the Center for High Performance Computing at VCU.

\section{Author contributions}

J.M.F., K.K.J., J.F.S. and G.A.B. comprised the executive committee. J.M.F., M.G.S., J.P.B., D.J.E., T.J.A., P.H.G., B.H., L.E., A.L.G., K.D.H.-M., K.K.J., J.F.S. and G.A.B. designed the study. P.H.G., S.C.V., S.H.M., S.K.R., M.R.D., A.L.S., M.G.G., C.E.R., N.R.W., K.D.H.-M., J.F.S. and J.M.F. comprised the clinical team. M.G.S., B.H., V.L., A.M.L., S.D.M., R.A.D., A.V.M., J.L.B., K.K.J., J.M.F. and G.A.B. generated the $16 \mathrm{~S} \mathrm{rRNA} /$ genomics/metagenomics data. M.G.S., D.J.E., B.H., J.X., S.J., A.M.L., J.I.D., R.A.D., J.M.F. and K.D.H.-M. generated the cytokine data. J.M.F., M.G.S., H.I.P., L.E., A.L.G., N.R.J., N.U.S., S.P.B., V.N.K., X.V.O., A.V.M. and G.A.B. managed the bioinformatics, genomics and data. J.P.B., D.J.E., H.I.P., T.J.A., S.S.F., Y.A.B., M.L.W., S.V.H., E.M.J., E.S. and J.M.F. did the statistical analysis and modeling. J.K., Y.-C.T. and J.M.F. did the TM7-H1 genome sequencing. C.E.R., M.G.G., D.O.C. and A.L.S. comprised the GAPPS team.

\section{Competing interests}

J.K. and Y.C.T. are full-time employees at Pacific Biosciences, a company developing single-molecule sequencing technologies. No other authors report any competing interests.

\section{Additional information}

Extended data is available for this paper at https://doi.org/10.1038/s41591-019-0450-2. Supplementary information is available for this paper at https://doi.org/10.1038/ s41591-019-0450-2.

Reprints and permissions information is available at www.nature.com/reprints. Correspondence and requests for materials should be addressed to G.A.B

Publisher's note: Springer Nature remains neutral with regard to jurisdictional claims in published maps and institutional affiliations.

(C) The Author(s), under exclusive licence to Springer Nature America, Inc. 2019

(c) (i) Open Access This article is licensed under a Creative Commons Attribution 4.0 International License, which permits use, sharing, adaptation, distribution and reproduction in any medium or format, as long as you give appropriate credit to the original author(s) and the source, provide a link to the Creative Commons license, and indicate if changes were made. The images or other third party material in this article are included in the article's Creative Commons license, unless indicated otherwise in a credit line to the material. If material is not included in the article's Creative Commons license and your intended use is not permitted by statutory regulation or exceeds the permitted use, you will need to obtain permission directly from the copyright holder. To view a copy of this license, visit http://creativecommons. org/licenses/by/4.0/. 


\section{Methods}

Participant enrollment, informed consent and health history collection. Participants for this study were enrolled from women visiting maternity clinics in Virginia and Washington State. All study procedures involving human subjects were reviewed and approved by the institutional review board at VCU (IRB no. HM15527). Participants were enrolled at multiple sites in Washington State by our partner registry, the GAPPS (see www.gapps.org) which was at the time under the umbrella of Seattle Children's IRB (FWA00002443), IRB Application number 12879. Study protocols were harmonized across sites, and data and samples from participants enrolled in Washington State were distributed to the VCU site. Samples were collected from ten maternal body sites (that is, vaginal, cervical, buccal and rectal mucosa, blood, urine, chest, dominant palm, antecubital fossa and nares), five types of birth products (that is cord blood, amniotic fluid, placental membranes, placental tissue and umbilical cord) and seven infant body sites (that is, buccal and rectal mucosa, meconium/stool, chest, right palm, nares and respiratory secretions if intubated). All study participants enrolled in Virginia and most participants enrolled at Washington State sites were also enrolled in the RAMS Registry at VCU. RAMS Registry protocols were approved at VCU (IRB no. HM15528); GAPPS-associated sites ceded review to the VCU IRB through reliance agreements. Additional samples were collected from the participants enrolled in Washington State, which are archived at the GAPPS. The present study was performed with compliance with all relevant ethical regulations. Written informed consent was obtained from all participants and parental permission and assent were obtained for participating minors aged at least 15 years.

Pregnant women were provided literature on the project and invited to participate in the study. Women who (1) were incapable of understanding the informed consent or assent forms or (2) were incarcerated were excluded from the study. Comprehensive demographic, health history and dietary assessment surveys were administered, and relevant clinical data (for example, gestational age, height, weight, blood pressure, vaginal pH, diagnosis) were recorded. Relevant clinical information was also obtained from neonates at birth and discharge.

At subsequent prenatal visits, triage, in labor and delivery, and at discharge, additional surveys were administered, relevant clinical data were recorded and samples were collected. Vaginal and rectal samples were not collected at labor and delivery or at discharge. Women with any of the following conditions were excluded from sampling at a given visit:

(1) Incapable of self-sampling due to mental, emotional or physical limitations

(2) More than minimal vaginal bleeding as judged by the clinician

(3) Ruptured membranes before 37 weeks

(4) Active herpes lesions in the vulvovaginal region

MOMS-PI PTB study case-control design. We initially selected 47 preterm cases of singleton, non-medically indicated PTBs from women who delivered between 23 weeks of gestation and 36 weeks and 6 days of gestation, and were selected from the 627 pregnancies in the Virginia arm of the study for whom gestational age at delivery was available at the time of the study design (phase 1 RAMS Registry cohort). From the phase 1 RAMS Registry cohort, 82 delivered before 37 weeks of gestation. Twelve of the participants who delivered preterm had multiple gestation pregnancies, twenty-one experienced medically indicated delivery, one delivered after fetal demise and one delivered a fetus at a non-viable gestational age. The participants had completed the study through delivery, and their gestational age information had been recorded in the study operational database at the time of the study design. We case matched the preterm participants 2:1 with participants who completed the study with singleton term deliveries $\geq 39$ weeks, to avoid complications associated with early term birth ${ }^{43-45}$, with matching based on ethnicity, age and annual household income. With these criteria, we matched controls to cases as closely as possible, loosening criteria at each pass using an in-house script; a few difficult-to-match cases were matched by hand. Case matching was performed blinded to all other study data. Two of the 47 PTBs did not have 16S rRNA that passed quality control, so these PTB samples and their controls were excluded from the MOMS-PI PTB study.

MOMS-PI TB study design. From an early subset of women who delivered in the MOMS-PI, we selected 90 pregnancies, including 41 women of European descent and 49 of African descent, who experienced term ( $\geq 39$ weeks of gestation) or early term birth (between 37 weeks of gestation and 38 weeks and 6 days of gestation).

Early pregnancy study design. An early pilot study was selected from a subset of 69 women in the MOMS-PI cohort from whom vaginal samples had been collected before 14 weeks of gestation. Targeted vaginal lipidomic profiles, vaginal cytokine profiles and vaginal $16 \mathrm{~S}$ rRNA taxonomic profiles were generated.

Maternal sampling schedule. Samples were collected from appropriately consented women at the enrollment visit, longitudinally at each prenatal visit, at triage, at labor and delivery, at discharge ( $24-48 \mathrm{~h}$ after birth) and at postpartum follow-up visits. All swab samples were collected with BD BBL CultureSwab EZ swabs. Self-sampling has been shown to provide samples equivalent to those collected by a trained clinician. Vaginal and rectal samples were collected either by healthcare providers during a pelvic exam (no speculum) or by self-sampling longitudinally throughout pregnancy and at postpartum follow-up visits. Vaginal and rectal samples were not collected at the discharge visit. Research coordinators instructed the participants on self-sampling procedures, provided a self-sampling instructional brochure and provided the participant a room for self-sampling. Maternal buccal samples were collected by a research coordinator during a study visit or by self-sampling at all visit types. Blood samples were collected at select visits by phlebotomists. Urine samples were longitudinally collected using a cleancatch protocol for a subset of participants. Cervical samples were collected by a clinician during a pelvic exam using a speculum at select visits. Samples from the antecubital fossa and nares were collected by research coordinators longitudinally throughout pregnancy, at discharge visits and at postpartum follow-up visits, but not at triage visits. At discharge visits, dominant palm and chest (skin) samples were also collected by a research coordinator. For samples collected at RAMS Registry sites, the following birth products were also collected: placental membranes, placental tissue, umbilical cord, cord blood and amniotic fluid (cesarean section only). Birth products, urine and additional blood samples for the participants enrolled through GAPPS sites are archived at the GAPPS Repository.

Infant sampling schedule. Samples from neonates (for example, buccal, rectal, meconium/stool, right palm, chest and nares) were collected at birth $(\sim 1 \mathrm{~h}$ after birth) and at discharge ( $24-48 \mathrm{~h}$ after birth). For infants admitted to the neonatal intensive care unit (NICU), additional samples were collected at days 3 and 7 of life and weekly until discharge from the NICU, or until the infant's first birthday. In the NICU, respiratory secretions were also collected from infants who were intubated. Buccal, rectal, right palm, chest and nares swabs were collected by healthcare providers. Meconium/stool samples were collected by research coordinators from diapers using sterile CultureSwab EZ swabs.

Swab sample preprocessing. Swab samples were collected as follows: (1) maternal mid-vaginal wall: a double-tipped CultureSwab EZ swab was inserted $\sim 5 \mathrm{~cm}$ into the vagina, pressed against the vaginal sidewall, rotated for $5 \mathrm{~s}$ and removed; (2) maternal cervical: during a speculum exam, a single-tipped CultureSwab EZ swab was inserted into the endocervix to the depth of the entire tip of the swab, rotated $360^{\circ}$, held for $10 \mathrm{~s}$ and removed, being careful not to contact the vaginal walls; (3) maternal and infant buccal: a double-tipped CultureSwab EZ was placed firmly in the mid-portion of the cheek, rotated for $5 \mathrm{~s}$ and removed; (4) maternal rectal: a double-tipped CultureSwab EZ swab was inserted to a depth of $\sim 2.5 \mathrm{~cm}$ into the rectum, rotated for $5 \mathrm{~s}$ and removed; (5) infant rectal: a single-tipped CultureSwab EZ swab was inserted to a depth of up to $\sim 0.64 \mathrm{~cm}$ into the rectum, rotated for $5 \mathrm{~s}$ and removed; maternal and infant nares samples were collected using single-tip CultureSwab EZ swab dipped in sterile saline and inserted $\sim 1.27 \mathrm{~cm}$ for maternal samples (up to $\sim 0.64 \mathrm{~cm}$ for infant) in the left nostril, rotated for $5 \mathrm{~s}$ and removed; (6) maternal and infant chest samples were collected using a double-tipped CultureSwab EZ swab dipped in sterile saline, pressed against the chest and rotated for $5 \mathrm{~s}$; maternal dominant palm and infant right palm samples were collected; (7) maternal dominant palm and infant right palm samples were collected using a double-tipped CultureSwab EZ swab dipped in sterile saline, pressed against the palm and rotated for $5 \mathrm{~s}$; (8) vaginal $\mathrm{pH}$ was collected using commercial applicators with $\mathrm{pH}$ paper. Briefly, the applicators were inserted $\sim 3.8-5 \mathrm{~cm}$ into the vagina, applied gently to the vaginal wall and withdrawn. The research coordinator compared the color of the $\mathrm{pH}$ indicator with a color chart and recorded the vaginal $\mathrm{pH}$.

Swabs were preprocessed and stored at $-80^{\circ} \mathrm{C}$ within an hour of collection. Material from swabs was transferred to the solutions compatible with downstream omics assays by vigorous spinning of swabs against the side walls of tubes for $15 \mathrm{~s}$. Maternal vaginal, maternal and infant buccal, maternal and infant rectal, maternal cervical, maternal and infant chest, maternal dominant palm, infant right palm and maternal antecubital fossa swabs for DNA isolation were immersed in $750 \mu \mathrm{l}$ MoBio PowerSoil DNA Isolation buffer; maternal vaginal, maternal and infant buccal, maternal and infant rectal, maternal cervical, maternal and infant chest, maternal dominant palm, infant right palm and maternal antecubital fossa swabs collected to be preserved for cultivation studies were immersed in $1 \mathrm{ml}$ of culture medium (brain heart infusion supplemented with $1 \%$ yeast extract, $2 \%$ gelatin, $0.1 \%$ starch, $1 \%$ glucose and $20 \%$ glycerol); maternal vaginal swabs for RNA purification were immersed in RNAlater (Qiagen); maternal vaginal and maternal and infant buccal swabs for cytokine profiling were immersed in $500 \mu \mathrm{l}$ of $10 \mathrm{mM}$ Tris, $\mathrm{pH}$ 7.0, $1 \mathrm{mM}$ ethylenediaminetetraacetic acid; maternal vaginal and maternal and infant buccal swabs were also immersed in $250 \mu \mathrm{l}$ of $10 \mathrm{mM}$ Tris, $\mathrm{pH} 7.0,1 \mathrm{mM}$ ethylenediaminetetraacetic acid; and maternal vaginal swabs were immersed in $500 \mu \mathrm{l}$ of $0.01 \%$ butylhydroxytoluene phosphate-buffered saline solution for lipid analyses.

Sample processing. DNA purification was performed using the MoBio PowerSoil Kit, as described by the manufacturer. RNA purification was performed using the MoBio PowerMicrobiome RNA Isolation Kit as described by the manufacturer. Total RNA was depleted of human and microbial rRNA using the Epicentre/ Illumina Ribo-Zero Magnetic Epidemiology Kit, as described by the manufacturer. DNA and RNA samples were stored at $-80^{\circ} \mathrm{C}$.

16S rRNA taxonomic surveys of the vaginal microbiome. DNA in each sample was amplified with barcoded primers targeting the V1-V3 region of the $16 \mathrm{~S}$ rRNA 
and validated for vaginal taxa as previously reported ${ }^{47}$. Primer sequences are listed in Supplementary Table 8. The samples were randomized at the PCR stage and again at the sequencing stage. Samples were multiplexed (384 samples per run) and sequenced on our Illumina MiSeq sequencer using 600 cycles creating $2 \times$ $300 \mathrm{bp}$ paired-end reads to generate a depth of coverage of at least 50,000 reads per sample. The raw sequence data were demultiplexed into sample paired-end fastq files based on unique barcode sequences using a customized Python script. The preprocessing of sequences was performed using the MeFiT ${ }^{62}$ pipeline, with amplicons (on average $~ 540$ base-pairs (bp) long) generated by merging the overlapping tails of paired-end sequences, followed by quality filtering using a MEEP (maximum expected error rate) cutoff of 1.0. Data were processed using harmonized bioinformatics pipelines with the other iHMP projects for upload to the Human Microbiome Project Data Coordination Center (HMP DACC). Nonoverlapping, high-quality reads were assigned to operational taxonomic units (OTUs), using the reference-based clustering method implemented by the pick closed_reference_otus.py script in the QIIME package. For data uploaded to the HMP DACC, the reference database used is the subset of Greengenes 16S rRNA sequence database clustered at $97 \%$ identity.

For each sample, the raw paired-end reads were uploaded to the HMP DACC and NIH Sequence Read Archive (SRA) as FASTQ files (16SRawSeqSet node), the quality-filtered reads as FASTQ files (16STrimmedSeqSet node) and the qiime output OTU tables in the biom format (16s_community).

For analysis of vaginal samples in the MOMS-PI PTB cohort, an alternate bioinformatics method was used. Non-overlapping, high-quality reads were screened for chimeric sequences with UCHIME ${ }^{63}$ against our custom database of vaginally relevant taxa. Each processed $16 \mathrm{~S}$ rRNA gene sequence was taxonomically classified to the species level using STIRRUPS ${ }^{47}$, which aligns against a custom reference database using USEARCH. Reference sequences for Prevotella cluster 2 include P. buccalis, . timonensis, Prevotella OTU46 and Prevotella OTU47. Only samples with at least 1,000 reads that met filtering criteria were analyzed. For vaginal samples, the STIRRUPS output tables were also uploaded to the HMP DACC (16s_community).

Whole shotgun metagenomic/metatranscriptomic sequencing. DNA libraries were prepared using KAPA Biosystems HyperPlus Library Kit and sequenced on our Illumina HiSeq $4000(2 \times 150 \mathrm{~b}$ PE). We sequenced all available vaginal samples for the PTB and TB cohorts, multiplexed 24 samples per lane and obtained $\sim 1-2 \times 10^{7} 150$-nucleotide reads per sample. For MGS, we subjected 555 vaginal samples to sequencing and 542/555 (97.7\%) had a minimum of 100,000 post-quality control (QC)-filtered read pairs. For MTS, we selected samples in the second trimester and the last collected sample for sequencing. If samples were not available in the second trimester, samples before and after the targeted second trimester window were selected. RNA was extracted from a total of 337 samples, of which $243(72.10 \%)$ had at least $10 \mathrm{ng} \mathrm{\mu l}^{-1}$ of RNA and were submitted for sequencing. Of these $242 / 243$ (99.6\%) met our minimum of 100,000 postQC-filtered read pairs. The rRNA-depleted messenger RNA was prepared for sequencing by constructing complementary DNA libraries using the KAPA Biosystems KAPA RNA HyperPrep Kit. Indexed complementary DNA libraries were pooled in equimolar amounts and sequenced on the Illumina HiSeq 4000 instrument, running four multiplexed samples per lane, with an average yield of $\sim 100 \mathrm{~Gb}$ per lane, sufficient to provide $>100 \times$ coverage of the expression profiles of the most abundant 15-20 taxa in a sample.

Whole shotgun metagenomic/metatranscriptomic data pre-processing. Raw sequence data were demultiplexed into sample-specific fastq files using $b c l 2 f a s t q$ conversion software from Illumina. Adapter residues were trimmed from both the $5^{\prime}$ - and the $3^{\prime}$-end of the reads using Adapter Removal tool v.2.1.3. The sequences were trimmed for quality using MEEPTOOLS ${ }^{64}$, retaining reads with a minimum read length of $70 \mathrm{~b}$ and MEEP quality score $<1$. Human reads were identified and removed from each sample by aligning the reads to the ho1 19 build of the human genome, using the BWA aligner. Taxonomic classification and relative abundance of bacteria in the metagenomes and metatranscriptomes, using harmonized bioinformatics pipelines with the other iHMP projects for upload to the HMP DACC, were obtained using Metaphlan2 (ref. ${ }^{65}$ ), with default parameters. The human-filtered MGS and MTS nodes were created at HMP DACC, WGSRawSeqSet and MicrobTranscriptomicsRawSeqSet, respectively, which link to the controlled-access data at the database of Genotypes and Phenotypes (study no. 20280). Metaphlan2 output community profiles of metagenomes and metatranscriptomes have been uploaded to HMP DACC, wgs_community node and microb_metatranscriptome node, as tab-delimited text files.

Functional analysis of metagenomic and metatranscriptomic sequencing reads. For vaginal samples in the MOMS-PI PTB study, assignment of MGS and MTS reads to known genes/pathways was performed using ASGARD ${ }^{66}$, HUMAnN2 (ref. ${ }^{67}$ ) and ShortBRED ${ }^{68}$. The reads were also compared with appropriate databases (KEGG, GO, COG, etc.) using BLAST or other alignment tools to characterize functional data about these samples. HUMAnN2 output functional profiles of metagenomes and metatranscriptomes have been uploaded to HMP DACC, $w g s_{-}$ functional node and microb_metatranscriptome node, as tab-delimited text files.
BVAB1 genome assembly from metagenomic reads. Starting with high-quality, trimmed, MGS reads from one sample with a high abundance of BVAB1, human reads were removed by alignment to the human hg19 reference genome using BWA alignment software. Human-filtered reads were digitally normalized with BBMap (https://sourceforge.net/projects/bbmap) with a target coverage of $>40 \times$ to remove reads from highly repetitive elements of the genomes that may hamper the de novo assembly process, and to ensure that reads originating from PCR duplication were excluded before assembly. Reads were assembled with SPAdes v.3.8.0 using the '-meta' option to generate a consensus assembly scaffold. Before clustering the scaffolds generated by SPAdes v.3.8.0, the human depleted reads were aligned back to the scaffolds using Bowtie 2 with the '--very-sensitive' option for global alignment. The resulting bam files were converted into 'scaffold-to-average coverage' maps using a customized Python script. These contigs were clustered into individual genomes using $\mathrm{MyCC}^{69}$, with tetramer frequencies coupled with the average coverage. Assembly identity was confirmed by alignment with $16 \mathrm{~S}$ rRNA sequences from BVAB1. Reads were mapped back to individual MyCC clusters and then submitted to a new assembly using Newbler Assembler v.2.8. Where necessary, gaps were closed by sequencing of PCR amplicons using primers directed to contig ends. Coverage of the final genome averaged over $40 \times$ and completion was confirmed by the presence of all 40 highly conserved marker genes commonly used to assess genome assemblies ${ }^{69}$. Genome sequences were annotated with in-house pipelines using Prokka and ASGARD ${ }^{66}$

TM7-H1 genome assembly from metagenomic reads using PacBio. DNA from a sample with high proportional abundance of TM7-H1 was sent to Pacific Biosciences for PacBio sequencing, using the TdT protocol, which is suitable for sequencing lowinput samples. An HGAP metagenome assembly was performed using a white list to exclude reads mapped to human, which yielded three TM7-H1 contigs. Genome sequences were annotated with in-house pipelines using Prokka and ASGARD ${ }^{66}$.

\section{Mapping of metagenomic and metatatranscriptomic sequencing reads to a} customized vaginal genome database representing 56 STIRRUPS taxa. We curated a custom database of vaginal genomes for 56 taxa identified in the 16S rRNA dataset that had a $0.1 \%$ average proportional abundance or for which at least $5 \%$ of samples were present at $0.1 \%$. We also included reference genomes for Chlamydia trachomatis and Neisseria gonorrhoeae. Reference genomes are not available for several STIRRUPS taxa that met one or both of these criteria: Bacteroides coagulans, Clostridiales BVAB2, Dialister cluster 51, Dialister propionicifaciens, Lachnospiraceae OTU33, Prevotellaceae OTU61 and Proteobacteria OTU-T1. An average of $95.2 \%$ of MGS reads from samples in the full-term cohort and $94.3 \%$ of MGS reads from samples in the preterm cohort were identified as human (see Extended Data Fig. 4). A significantly smaller percentage of MTS reads (that is, an average of $30.0 \%$ and $32.8 \%$ in the full-term and preterm cohorts, respectively) were identified as human. This likely reflects the sampling of dead or dying vaginal epithelial cells. Bowtie2 default parameters were used to map filtered, non-human-read MTS and MGS reads to the customized vaginal genome database. All genomes were reannotated using Prokka. MacSysFinder was used to identify genes involved in bacterial secretion systems in the genomes in the reference database ${ }^{70}$. FeatureCounts was used to count paired-end reads where both ends mapped to non-ribosomal genes (coding sequences, transfer RNAs and transfer-messenger RNAs). Ribosomal genes were not included because we were interested in testing candidate taxa using other genes to build confidence that identification of candidate taxa was not dependent on $16 \mathrm{~S}$ rRNA microbiome-profiling protocol choices. Ribosomal genes were thus classified as unassigned, no features. Chimeras were excluded, only primary alignments were mapped and duplicate reads were excluded. Paired MGS and MTS data were available for 41 women who delivered preterm and 81 term controls.

To confirm that the microbial signatures of PTB identified using 16S rRNA data were not attributable to protocol choices or protocol biases, we used MGS and MTS data to support these findings. The MGS data and 16S rRNA profiles were typically generated from the same DNA preparations, but they used independent sequencing strategies and bioinformatics and analysis methods. The MTS data were generated from vaginal swab samples collected in parallel with those used for DNA extractions, but the downstream protocol was entirely different. We analyzed one time point per participant, with a mean gestational age of sampling at 25 weeks for the preterm cohort and 26 weeks for the full-term cohort, and used a global scaling approach to normalize to all genes in the 56 taxa in our database. DESeq2 (ref. ${ }^{71}$ ) was used to compare term and preterm cohorts using an organismindependent, global-scaling approach; genes with fewer than 1,000 total mapped genes across samples were excluded from analysis. Note that, with global scaling, it is not possible to differentiate differences caused by differential abundance and those due to differential expression in MTS data. Thus, read counts mapping to a bacterial gene from a specific taxon were normalized to the total number of reads mapping to all bacterial genes in the database.

Given that our MTS results reflect findings from 16S rRNA microbiome analyses, the results are most likely largely driven by differential abundance between term and preterm cohorts. For comparative analyses across $16 \mathrm{~S}$ rRNA, MGS and MTS, the proportional abundance was calculated based on the 56 taxa that could be measured across all three approaches. To calculate the taxonomic proportional abundance for an MGS or MTS sample, the sum of reads mapped 
to genes for a given taxon was divided by the total number of reads mapped to all bacterial genes in the database. For this analysis, the 16S rRNA counts for a sample were renormalized by dividing the total number of reads mapped to a taxon by the total reads mapped to all taxa represented in the custom genome database, to which the MGS and MTS samples were mapped.

Metabolic modeling. Draft constraint-based metabolic models ${ }^{72}$ for TM7-H1, BVAB1 and L. crispatus were generated using functional annotation information with Enzyme Commission numbers to describe function and KEGG IDs for nomenclature. Model contents are provided in Supplementary Information File 6 as .xls files and mapping files are provided as .xml files. Draft models have not been gap filled, given that most of these organisms are poorly characterized, and previously studied species of TM7 have been found to be non-free living.

MOMS-PI PTB study: cytokine profiling. The Bio-Plex Pro Human Cytokine 27-plex Assay panel (M50-0KCAF0Y, Bio-Rad) was used to measure cytokine concentrations according to the manufacturer's protocol. Briefly the frozen vaginal swab samples were thawed on ice and centrifuged at $10,000 \mathrm{~g}$ for $10 \mathrm{~min}$ at $4{ }^{\circ} \mathrm{C}$ and diluted fourfold in $100 \mathrm{mM}$ Tris buffer, $\mathrm{pH}$ 7.5. The assay was carried out on a black 96 -well plate (10021013, Bio-Rad), and $50 \mu$ l of cytokine standard, interassay QC (described below) and sample were added in duplicate to appropriate wells. The Bio-Plex MAGPIX Multiplex Reader was used for data acquisition with default settings. Bio-Plex Manager v.6.0 software was used for data analysis using five-parameter logistic (5-PL), non-linear regression model on optimization for all analytes within $70-130 \%$ of the recovery range.

The interassay QC control was prepared from lipopolysaccharide-stimulated cell culture medium. Briefly, VK2/E6E7 (American Type Culture Collection (ATCC) CRL-2616) cells were initially grown in T75 flasks in Dulbecco's modified Eagle's medium/F-12 supplemented with 10\% FBS (11320-033, 26140079, ThermoFisher) at $37^{\circ} \mathrm{C}, 5 \% \mathrm{CO}_{2}$ to confluency. These cells were trypsinized and reseeded at a concentration of $3 \times 10^{5}$ cells ml $^{-1}$ per well on a 24 -well plate ( 82050 892 , VWR). After $24 \mathrm{~h}$, the medium was replaced with the fresh medium containing $100 \mathrm{ng} \mathrm{ml}^{-1}$ lipopolysaccharide (L2630-10MG, Sigma). Twenty-four hours postlipopolysaccharide treatment, the cell culture medium was harvested, pooled and centrifuged at $3,000 \mathrm{~g}$ for $10 \mathrm{~min}$ at $4{ }^{\circ} \mathrm{C}$. The resultant soluble fraction was aliquoted and stored at $-80^{\circ} \mathrm{C}$ for use as assay QC. Out-of-range cytokine concentration values were imputed with the upper or lower limit of detection for the specific cytokine where necessary. Nine cytokines (that is, IL-1 $\beta$, eotaxin, IL- 8 , TNF- $\alpha$, IL-17A, MIP-1 $\beta$, IL-6, IP-10/CXCL10 and RANTES) had fewer than $30 \%$ out-ofrange values and were selected for analysis. Cytokine concentrations normalized per swab sample were uploaded to HMP DACC (host_cytokine node) as tab-delimited text files. Cytokine data from the MOMS-PI PTB study, the MOMS-PI TB study and the MOMS-PI Early Pregnancy study were not designed to be used in concert due to differences in lots of reagents and normalization protocols. Sub-study tags have been uploaded to the HMP DACC to discriminate samples by study.

MOMS-PI TB study: cytokine profiling. The Bio-Rad Bio-Plex Pro Human Cytokine 27-Plex Assay was employed with a Luminex 100/200 System to quantify cytokine levels in the vaginal samples. Frozen vaginal swab samples suspended in $500 \mathrm{\mu l} 100 \mathrm{mM}$ Tris- $\mathrm{HCl}, \mathrm{pH} 7.5$, were thawed on ice and centrifuged at $10,000 \mathrm{~g}$ for $10 \mathrm{~min}$ at $4^{\circ} \mathrm{C}$. The Bio-Plex assay was conducted on samples and serial dilutions of standards in duplicate, according to the manufacturer's instructions. Values were analyzed using a 5-PL, non-linear regression curve model. Cytokine values deemed out of range were assigned the upper or lower limit of detection for the specific cytokine. Cytokine concentrations were uploaded to the HMP DACC (host_cytokine node) as tab-delimited text files. The values at the DACC were not normalized to protein concentration as determined by the Bradford Assay ${ }^{39}$. Cytokine data from the MOMS-PI PTB study, the MOMS-PI TB study and the MOMS-PI Early Pregnancy study were not designed to be used in concert due to differences in lots of reagents and normalization protocols. Sub-study tags have been uploaded to the DACC to discriminate samples by study.

MOMS-PI Early Pregnancy study: cytokine profiling. The Bio-Rad Bio-Plex Pro Human Cytokine 27-Plex Assay was employed with a Luminex 100/200 System to quantify cytokine levels in the vaginal samples. Frozen vaginal swab samples suspended in $500 \mu \mathrm{l} 100 \mathrm{mM}$ Tris- $\mathrm{HCl}, \mathrm{pH} 7.5$, were thawed on ice and centrifuged at $10,000 \mathrm{~g}$ for $10 \mathrm{~min}$ at $4^{\circ} \mathrm{C}$. The Bio-Plex assay was conducted on samples and serial dilutions of standards in duplicate, according to the manufacturer's instructions. Values were analyzed using a 5-PL, non-linear regression curve model. Cytokine values deemed out of range were assigned the upper or lower limit of detection for the specific cytokine. IL-2, IL-5 and IL-15 levels were below the limit of detection in $>50 \%$ vaginal samples and were not included in subsequent analyses. Although biological replicates could not be performed due to the effects of multiple freezethaw cycles on sample viability, the intra-assay coefficient of variation was found to be acceptable at $<7 \%$ for all cytokines. Cytokine concentration $\left(\mathrm{pg} \mathrm{ml}^{-1}\right)$ was divided by total protein concentration $\left(\mathrm{mg} \mathrm{ml}^{-1}\right)$ to yield normalized cytokine concentration ( $p g$ cytokine mg protein ${ }^{-1}$ ) for each sample. Samples for which total protein could not be determined were not included in the analysis. Cytokine concentrations were uploaded to the HMP DACC (host_cytokine node) as tab-delimited text files. The values at the DACC were not normalized to protein concentration as determined by the Bradford Assay ${ }^{39}$. Cytokine data from the MOMS-PI PTB study, the MOMS-PI TB study and the MOMS-PI Early Pregnancy study were not designed to be used in concert due to differences in lots of reagents and normalization protocols. Sub-study tags have been uploaded to the DACC to discriminate samples by study.

Early Pregnancy study: lipidome profiling of vaginal samples. A pilot study was performed for lipidome profiling of vaginal samples. For eicosanoid and sphingolipid quantification, an equal volume of ethanol containing $10 \mathrm{ng}$ of eicosanoid internal standards or methanol containing $50 \mathrm{pmol}$ of sphingolipid internal standards was added to clarified vaginal swab contents dispersed in phosphate-buffered saline containing $0.01 \%$ butylhydroxytoluene. Eicosanoid internal standards consisted of 30 deuterated analytes, including $\left(d_{4}\right) 6$-ketoprostaglandin (PG)F-1 $\alpha,\left(d_{4}\right)$ PGF- $2 \alpha,\left(d_{4}\right)$ PGE-2, $\left(d_{4}\right)$ PGD-2, $\left(d_{4}\right)$ LTB $_{4}$ (leukotriene $\left.\mathrm{B}_{4}\right),\left(d_{4}\right)$ thromboxane $(\mathrm{Tx}) \mathrm{B}_{2},\left(d_{4}\right) \mathrm{LTC}_{4},\left(d_{5}\right) \mathrm{LTD}_{4},\left(d_{5}\right) \mathrm{LTE}_{4}$, $\left(d_{8}\right)$ 5-hydroxyeicosatetranoic acid, $\left(d_{8}\right)$ 15-hydroxyeicosatetranoic acid, $\left(d_{8}\right)$ 14,15-epoxyeicosatrienoic acid, $\left(d_{8}\right)$ arachidonic acid and $\left(d_{5}\right)$ eicosapentaenoic acid. Sphingolipid internal standards consisted of $d_{17}$ sphingosine, sphinganine, sphingosine-1-phosphate, sphinganine-1-phosphate and $d_{\text {18:1/12:0 }}$ ceramide-1phosphate, sphingomyelin, ceramide and monohexosylceramide (Avanti). After centrifugation at $12,000 \mathrm{~g}$ for $20 \mathrm{~min}$, the resultant mixture was subjected to UPLC electrospray ionization mass spectrometry/mass spectrometry analysis using a hybrid, triple quadrupole, linear ion trap mass analyzer (ABSCIEX 6500 QTRAP) via multiple-reaction monitoring. Detailed separation, elution and ionization conditions have been previously described ${ }^{73}$ and are summarized in the Supplementary Appendix 1. Spectral data were analyzed using MultiQuant software (ABSCIEX) and quantification was carried out by comparison against known quantities of internal standards and a seven-point dilution curve. The values were uploaded as tab-delimited text files to the HMP DACC. The values at the DACC were not normalized to protein concentration as determined by the Bradford Assay ${ }^{39}$. Cytokine data from the MOMS-PI PTB study, the MOMS-PI TB study and the MOMS-PI Early Pregnancy study were not designed to be used in concert due to differences in lots of reagents and normalization protocols. Substudy tags have been uploaded to the DACC to discriminate samples by study.

Community state types/vagitypes. Vaginal $16 \mathrm{~S}$ rRNA profiles were assigned to community state types (CSTs) based on the taxon with the largest proportion of reads. Samples in which the largest proportion was less than $30 \%$ were not assigned a CST/vagitype. This 'predominant taxon' rule has been shown to exhibit over $90 \%$ agreement with clustering-based methods across a variety of vaginal microbiome datasets $^{46}$, and yet is not population or dataset dependent and is therefore more conducive to use in a clinical setting. Differences in the numbers of $L$. crispatus CSTs among the PTB and TB cohorts were tested using a Fisher's exact test.

Markov chain analysis. The R package $m s m$ was used to fit a continuous-time Markov chain model for CST transitions. The model takes as input the subject, $\mathrm{CST} /$ vagitype and gestational age in days for each sample. The states were L. crispatus, L. iners, BVAB1, G. vaginalis and 'Other'. The pregnancy outcome (that is, preterm or term birth) was included as a covariate. To derive confidence intervals for maximum likelihood estimates of transition probabilities, the set of transitions modeled was restricted to those in which at least four transitions were observed between subsequent visits for individuals in the study. Without this restriction, the maximum likelihood method does not converge. A stationary distribution for each group was estimated by setting the time to 100,000 days. Dynamic balance was checked for the one-trimester transition probabilities by taking the difference between the forward and reverse transition probabilities.

Filtering out low-abundant taxa. As the first step in analyzing each dataset of vaginal 16S rRNA profiles, we analyzed the abundance of each taxa present in the profiles, and removed from further consideration low-abundant species. We used two abundance criteria: we retained taxa that either (1) $5 \%$ of the profiles exhibited an abundance of at least $1 \%$, or (2) at least $15 \%$ of profiles exhibited an abundance of at least $0.1 \%$. Taxa that failed to meet both (1) and (2) were removed.

Univariate analysis to identify taxa very different in abundance in PTB and TB cohorts. We analyzed vaginal $16 \mathrm{~S}$ rRNA profiles from 135 participants, 45 who delivered preterm and 90 who delivered term. The microbiome profile of the earliest sample from each of these women was used in this analysis. In this dataset, 26 taxa remained after filtering out the low-abundance taxa. For each of these 26 most abundant taxa, we performed a Mann-Whitney $U$-test to identify important differences in the presence and abundance in PTB and TB cohorts. For this analysis, abundance values below 0.00001 were rounded to zero. Taxa abundance was considered greatly different between cohorts if the $q$ value was less than an FDR of $5 \%$ after correction via the Benjamini-Hochberg procedure. For each taxon we also calculated the median and the 75th percentile in the PTB and TB cohorts.

Longitudinal models. A GAMM ${ }^{74}$ incorporating BMI, ethnicity (African, European), pregnancy outcome (preterm, full term), a smoother for gestational age and a random subject effect was used to longitudinally model log-transformed 
relative abundances of vaginally relevant taxa. Effect contributions were determined using analysis of variance tests. The model is described by the formula:

$\log (\text { Abundance })_{i j}=\beta_{0}+\beta_{1} \mathrm{PO}_{I}+\beta_{2} \mathrm{BMI}_{i j}+\beta_{3} \mathrm{pH}_{i j}+\beta_{4} I_{\text {ethnicity }}+f\left(\mathrm{ga}_{i j}\right) \times \mathrm{PO}_{i}+\gamma_{I}$ $+\varepsilon_{i j}$

where $\log (\text { Abundance })_{i j}$ is the log-transformed taxa relative abundance for the $j$ th observation of the $i$ th subject, $\mathrm{PO}$ is pregnancy outcome (preterm, full term), $\mathrm{pH}$ is vaginal $\mathrm{pH}, I_{\text {ethnicity }}$ is an indicator variable that takes on the value 0 for subjects of African ancestry and 1 for subjects of all other ancestries, ga is gestational age, $f($.) is a smooth function, $\gamma_{i}$ is the random effect for the $i$ th subject and $\varepsilon_{i j}$ is the error term.

The degree of smoothness for gestational age was estimated by restricted maximum likelihood ${ }^{75}$. Models were fit using the gamm4: GAMMs using mgcv and lme4 package in R, released by Wood and Scheipl in 2017.

Canonical correlation analysis of cytokines and vaginal microbiome profiles. An integrative analysis of both log-transformed 16S rRNA survey data and log-transformed cytokine data was performed using $\mathrm{sCCA}^{76}$. Classic canonical correlation analysis ${ }^{77}$ explores the correlation between two sets of quantitative variables measured on the same subjects. The sCCA introduces an 11-penalization term to handle the case of more variables than observations. Nine cytokines, with fewer than $30 \%$ out-of-range values, were selected for analysis (that is, IL- $1 \beta$, eotaxin, IL-8, TNF- $\alpha$, IL-17A, MIP-1 $\beta$, IL-6, IP-10/CXCL10, RANTES). Out-ofrange cytokine concentration values were then imputed with the upper or lower limit of detection for the specific cytokine where appropriate. For each subject, the observation corresponding to the earliest gestational age per trimester was used for analysis. We performed sCCA separately for full-term and preterm subjects using the sgcca function in the $\mathrm{R}$ package mixOmics ${ }^{78}$.

The results of sCCA are displayed in a correlation circle plot $^{77}$. The coordinates of the plotted points (variables) are the correlations between the variables and their canonical variates. Variables that have a strong positive correlation are projected close to each other on the plot, whereas variables that are negatively correlated are plotted opposite each other. The greater the distance from the origin, the stronger the relationship among variables ${ }^{77}$. The correlation circle plots are constructed using the plotVar function in the $\mathrm{R}$ package mixOmics.

Taxon co-occurrence. Bacterial taxa were determined to be present if they comprised $\geq 0.1 \%$ of the total vaginal microbiome profile. We utilized the statistical tool $\mathrm{REBACCA}^{79}$ to mitigate the effects of relative constraint. REBACCA was run using 50 bootstraps and a visualization of bacterial correlations was generated using Gephi. Correlations with more than 0.3 or less than -0.3 are shown, with negative correlations in red and positive correlations in blue. Edge weights are representative of the strength of correlation between taxa and the four major predictive taxa, shown in gray.

Predictive modeling of PTB using early pregnancy microbiome profiles. We constructed a linear predictive model of PTB as follows: from the full cohort, we selected subjects who had at least one vaginal $16 \mathrm{~S}$ rRNA sample early in the pregnancy, in days 42-167 (inclusive) of gestation. A total of 31 PTB and 59 TB subjects had at least one sample in this time window; if multiple samples were present in that window, we used the earliest sample.

We first filtered out low-abundant species in this dataset: 25 passed the selection criteria. For these taxa, the abundance data were soft-thresholded with a 0.001 threshold, to reduce the impact of statistical noise resulting from low-abundance values, by subtracting 0.001 from the abundance and setting all resulting negative values to 0 , and log-transformed through a transform $\log _{10}(($ abundance +0.001$) / 0.001)$, where dividing by 0.001 shifts the logarithm values for abundances in the zero (0.0) to 1 (1.0) range from negative to non-negative values. Ten taxa were significantly different between the PTB and TB cohorts.

The model construction uses a two-step procedure: first, we applied a MannWhitney $U$-test to all species that survived the abundance-based filtering criteria, retaining species with a two-sided $P$ value of 0.05 or less. Based on these species, the predictive model was trained using logistic regression with $\mathrm{L}_{1}$ regularization ${ }^{80}$, to reduce the impact of collinearity between species and the resulting sign reversals and false detections. Regularized logistic regression finds a vector of taxa weights w that minimizes: $\Sigma_{i} \ln \left(1+e^{\left(-y_{i} w^{T} x_{i}\right)}\right)+C\|\mathbf{w}\|_{1}$ over the training set of samples $\left(\boldsymbol{x}_{\boldsymbol{i}}, y_{i}\right)$. The constant $\mathrm{C}$ was selected based only on samples from the training set, using grid search and nested cross-validation.

The statistical significance of the model in the form of a $P$ value was estimated using a permutation test, consisting of training 10,000 models on data with the class variable randomly permuted before processing, and comparing the distribution of the 10,000 AUROC values with the AUROC values of the original model trained using unperturbed class variable. Performance of the model on previously unseen samples was assessed using the leave-one-out method. We assessed sensitivity, specificity and AUROC, as implemented in the Python scikitlearn package ${ }^{81}$
The PTB predictive score was defined as: $0.775 \log _{10}(\mathrm{Samn})+0.751$ $\log _{10}(\mathrm{BVAB} 1)+0.116 \log _{10}(\mathrm{TM} 7)+0.011 \log _{10}(\mathrm{Pcl} 2)$. To avoid negative values of the logarithm for abundance data on the $[0,1]$ scale, we used the logarithm in the form $\log _{10}(($ abundance +0.001$) / 0.001)$, where abundance is soft-thresholded at 0.001 , that is, 0.001 is subtracted from abundance values, and negative values are replaced by 0 . This transformation shifts $\log$ (abundance) from the negative $[-3,0]$ range to the non-negative [0-3] range, but does not affect the relative positions of the samples.

Predictive modeling of PTB using clinical variables. For comparison with the microbiome-based predictive model, we also trained a predictive model using only clinical variables, without any input from the subject's microbiome. We defined the following 11 clinical variables: short cervix: (YES/NO), cerclage (YES/NO), vaginal $\mathrm{pH}, \mathrm{BMI}$, progesterone (YES/NO), gravidity, parity, gravidity minus parity, history of miscarriage or stillbirth (YES/NO), history of PTB (YES/NO), use of antibiotics in 6 months before sampling. YES/NO features were converted to $1=\mathrm{YES}, 0=\mathrm{NO}$. Other features were normalized to be in the $0-1$ range by subtracting the minimal value, and then dividing by the difference between minimum and maximum in the dataset. The training of the clinical information-based predictive model operates in two stages, as with the microbiome-based model. First, features are filtered through a univariate Mann-Whitney $U$-test. Second, a regularized linear model is trained using features that remain after filtering. The leave-one-out estimates of the predictive power of the clinical information-based model are: AUROC 0.764, sensitivity $69.5 \%$, specificity $74.2 \%$.

Cross-study comparison cohort selection. We performed a systematic literature review to identify previous studies that used taxonomic markers to assess the vaginal microbiome and $\mathrm{PTB}^{22-27,30-37,57}$. Three studies included cohorts of pregnant women who were predominantly of African descent, were at high risk for PTB and had publicly available $16 \mathrm{~S}$ rRNA reads ${ }^{25,27,30}$ (bioProjects: PRJNA242473, PRJNA294119, PRJNA393472-(University of Alabama (UAB) cohort). De-identified clinical data for bioProject PRJNA294119 was kindly made available by Molly Stout. There was substantial variation in the inclusion and exclusion criteria for the case and control groups across studies. For example, Stout et al. ${ }^{27}$ excluded subjects taking supplemental progesterone from the study, whereas, in contrast, Callahan et al..$^{25}$ included only subjects receiving supplemental progesterone in the UAB high-risk cohort. The inclusion criteria for PTB differed substantially; the study by Romero et al..$^{30}$ included only participants who experienced spontaneous labor or preterm premature rupture of membranes and delivered before 34 weeks. In contrast, participants with indicated PTB (for example, pre-eclampsia) accounted for most PTBs in the Stout et al. ${ }^{27}$ study. There were similar differences in the control groups across studies. There were critical differences across all aspects of experimental design: sample collection, hypervariable region(s) of 16S rRNA examined, primer selection and PCR, sequencing technology and bioinformatics pipelines.

Cross-study comparison cohort matching. In the present study, the selected cases were all spontaneous PTBs that occurred before 37 weeks of gestation; early term births (that is, those delivered between 37 weeks and 38 weeks 6 days) were excluded from the controls. To identify the most suitable replication cohorts available for the present study, we reanalyzed data generated from a subset of samples collected from women who experienced spontaneous PTB before 37 weeks of gestation and matched term controls delivered at a gestational age of 39 weeks or later. Only cases/controls with at least one sample, with a minimum of 1,000 reads after trimming and quality filtering, were included. Controls were matched $2: 1$ to cases from the same original study, based on the matching criteria used in the present study, as far as possible. This included ancestry/ethnicity for all three studies and age for two of the studies. Age was not available the Stout et al. study ${ }^{27}$ (that is, PRJNA294119). Annual household income was not used for matching because it was not available for any of the three studies. In the high-risk cohort from the Callahan et al..$^{25}$ study (that is, PRJNA393472-UAB cohort), there was not a sufficient number of controls who met the criteria, and therefore cases for the available controls to preserve the matching ratio. For the Romero et al. ${ }^{16}$ study, there was an excess of available controls, so we restricted selection of controls to those who had a sample collected before 37 weeks of gestation. Selection of rematched cases/controls was performed blinded to other data.

Replication cohort processing of $16 S$ rRNA data. We applied a standardized bioinformatics pipeline to the $16 \mathrm{~S}$ rRNA reads from the three original studies to harmonize with the present study as far as possible. Reads were downloaded from the SRA (bioProjects: PRJNA242473, PRJNA294119, PRJNA393472-UAB cohort). Cutadapt was used to trim adapters and primers, if present. We applied harmonized quality filtering across the studies. For studies using the 454 Roche sequencing technology, we applied filtering criteria established by our group in the Vaginal Human Microbiome Project which has been successfully used with our established STIRRUPS taxonomic classification ${ }^{47}$ and statistical analyses. Thus, we trimmed from the $3^{\prime}$-end of the read until the average quality score of the last $10 \mathrm{bp}$ was 20 or more. If the resulting read was less than $200 \mathrm{bp}$ in length, it was discarded. For the Stout et al..$^{27}$ study, we analyzed only the V1-V3 reads. For the Callahan et al. ${ }^{2.5}$ study, the reads were trimmed and quality filtered, and forward and reverse reads 
were merged before upload to the SRA. The quality filtering criteria used for the Illumina HiSeq data in that original study (that is, each read of $235 \mathrm{bp}$ or $245 \mathrm{bp}$ needed to have fewer than two expected errors) was consistent with the filtering criteria applied to the Illumina MiSeq data in the present study (that is, MEEP score cutoff of 1). The forward and reverse reads both covered the V4 region, so we used only the forward reads from that study for taxonomic analysis. For all studies, only samples with a minimum of 1,000 quality-filtered reads were used in downstream analyses. A total of 85 of the 149 samples in the Stout et al. study met this QC threshold and 348 of 349 samples in the Romero et al. ${ }^{16}$ study. All 1,280 samples from the Callahan et al. UAB cohor $\mathrm{t}^{25}$ had a minimum of 1,000 quality-filtered reads. Clinical data were available for two additional samples in the Callahan et al. UAB cohort for which sequence data were not available at the SRA.

Cross-study comparison cohort taxonomic classification. Although the primers used in the Stout et al. ${ }^{27}$ and Romero et al. ${ }^{16}$ studies were different from each other and those used in the present study, the same V1-V3 hypervariable region was amplified. Thus, the trimmed quality-filtered reads from the Romero et al. ${ }^{30}$ and the Stout et al..$^{27}$ studies were run through the STIRRUPS pipeline, using the same database as the present study. We do not have a curated custom database of vaginally relevant taxa for the $\mathrm{V} 4$ hypervariable region. Thus, we curated a small V4 database (https://github.com/Vaginal-Microbiome-Consortium/PTB) corresponding to our V1-V3 database for the taxa identified as very different between cases and controls in the present study. We used the STIRRUPS pipeline to align reads from the Callahan et al. UAB cohort ${ }^{25}$ against this small V4 database with taxa of interest.

Notably, in the Stout et al. replication cohort, all four taxa used in our predictive model (S. amnii, BVAB1, TM7-H1, Prevotella cluster 2) had higher abundance in the PTB group than in the TB group. Moreover, for 8 of the 14 taxa identified as differing between the PTB and TB groups in the analyses of our cohort, the difference in medians between the PTB and TB groups in the Stout cohort occurred with the same directionality as in our cohort. Of the remaining six taxa, four were infrequent and had medians below the abundance cutoff threshold in both TB and PTB groups of the Stout et al. cohort (that is, TM7-H1, BVAB2, Dialister micraerophilus, $P$. amnii), but they still had a higher abundance range in PTB than in TB groups, as in our cohort. Only Achr and Dcl51 showed an opposite trend of medians in the Stout et al. cohort compared with ours. In the Callahan et al. replication cohort, three of the four taxa used in our predictive model had a higher median in the PTB group than in the TB group, similar to our cohort. Moreover, in 11 of 14 taxa, the median between the PTB and TB groups in the Callahan et al. cohort differed in the same direction as in our cohort. TM7 had a median below the cutoff threshold. Only Dialister cluster 51 and Megasphaera type 1 OTU70 showed an opposite trend in the medians of the Callahan et al. cohort compared with ours.

Nucleotide sequence accession numbers. The genome sequences of BVAB1 S1 (PQVO000000) and TM7-H1 E1 (CP026537) are available at the GenBank database.

Reporting Summary. Further information on research design is available in the Nature Research Reporting Summary linked to this article.

\section{Data availability}

Open-access data including raw $16 \mathrm{~S}$ rRNA sequences, cytokine data and limited metadata are available at the HMP DACC (https://portal.hmpdacc.org). Controlled-access data including raw MGS data, raw MTS data and metadata for all subjects analyzed in this study are available at National Center for Biotechnology Information's controlled-access dbGaP (study no. 20280; accession ID phs001523. v1.p1) and the SRA under BioProject IDs PRJNA326441, PRJNA326442 and PRJNA326441. The genomes of TM7-H1 (CP026537) and BVAB1 (PQVO000000) have been submitted to GenBank. Access to additional fields can be requested through the RAMS Registry (https://ramsregistry.vcu.edu). Additional project information is available at the project's website (http://vmc.vcu.edu/momspi).

\section{Code availability}

The custom code is available at https://github.com/Vaginal-MicrobiomeConsortium/PTB.

\section{References}

62. Parikh, H. I., Koparde, V. N., Bradley, S. P., Buck, G. A. \& Sheth, N. U. MeFiT: merging and filtering tool for illumina paired-end reads for $16 \mathrm{~S}$ rRNA amplicon sequencing. BMC Bioinforma 17, 491 (2016).

63. Edgar, R. C., Haas, B. J., Clemente, J. C., Quince, C. \& Knight, R. UCHIME improves sensitivity and speed of chimera detection. Bioinforma 27, 2194-2200 (2011)

64. Koparde, V. N., Parikh, H. I., Bradley, S. P. \& Sheth, N. U. MEEPTOOLS: a maximum expected error based FASTQ read filtering and trimming toolkit. Int. J. Comput. Biol. Drug Des. 10, 237-247 (2017).

65. Segata, N. et al. Metagenomic microbial community profiling using unique clade-specific marker genes. Nat. Methods 9, 811-814 (2012).

66. Alves, J. M. P. \& Buck, G. A. Automated system for gene annotation and metabolic pathway reconstruction using general sequence databases. Chem. Biodivers. 4, 2593-2602 (2007).

67. Langille, M. G. I. et al. Predictive functional profiling of microbial communities using 16S rRNA marker gene sequences. Nat. Biotechnol. 31, 814-821 (2013)

68. Kaminski, J. et al. High-specificity targeted functional profiling in microbial communities with ShortBRED. PloS Comput. Biol. 11, e1004557 (2015).

69. Lin, H.-H. \& Liao, Y.-C. Accurate binning of metagenomic contigs via automated clustering sequences using information of genomic signatures and marker genes. Sci. Rep. 6, 24175 (2016).

70. Abby, S. S. et al. Identification of protein secretion systems in bacterial genomes. Sci. Rep. 6, 23080 (2016)

71. Love, M. I., Huber, W. \& Anders, S. Moderated estimation of fold change and dispersion for RNA-seq data with DESeq2. Genome Biol. 15, 550 (2014).

72. Fong, S. S. Computational approaches to metabolic engineering utilizing systems biology and synthetic biology. Comput. Struct. Biotechnol. J. 11, 28-34 (2014).

73. Simanshu, D. K. et al. Non-vesicular trafficking by a ceramide-1-phosphate transfer protein regulates eicosanoids. Nature 500, 463-467 (2013).

74. Lin, X. \& Zhang, D. Inference in generalized additive mixed modelsby using smoothing splines. J. R. Stat. Soc. Ser. B 61, 381-400 (1999).

75. Harville, D. Maximum likelihood approaches to variance component estimation and to related problems. J. Am. Stat. Assoc. 72, 320-338 (1977).

76. Witten, D. M., Tibshirani, R. \& Hastie, T. A penalized matrix decomposition, with applications to sparse principal components and canonical correlation analysis. Biostatistics 10, 515-534 (2009).

77. González, I., Cao, K.-A. L., Davis, M. J. \& Déjean, S. Visualising associations between paired 'omics' data sets. BioData Min. 5, 19 (2012).

78. Rohart, F., Gautier, B., Singh, A. \& Lê Cao, K.-A. mixOmics: an R package for 'omics feature selection and multiple data integration. PLoS Comput. Biol. 13, e1005752 (2017)

79. Ban, Y., An, L. \& Jiang, H. Investigating microbial co-occurrence patterns based on metagenomic compositional data. Bioinforma 31, 3322-3329 (2015).

80. Ng, A. Y. Feature selection, L1 vs. L2 regularization, and rotational invariance. In Proc. 21st International Conference on Machine Learning 78 (ACM, 2004); https://doi.org/10.1145/1015330.1015435

81. Pedregosa, F. et al. Scikit-learn: machine learning in Python. J. Mach. Learn. Res. 12, 2825-2830 (2011). 

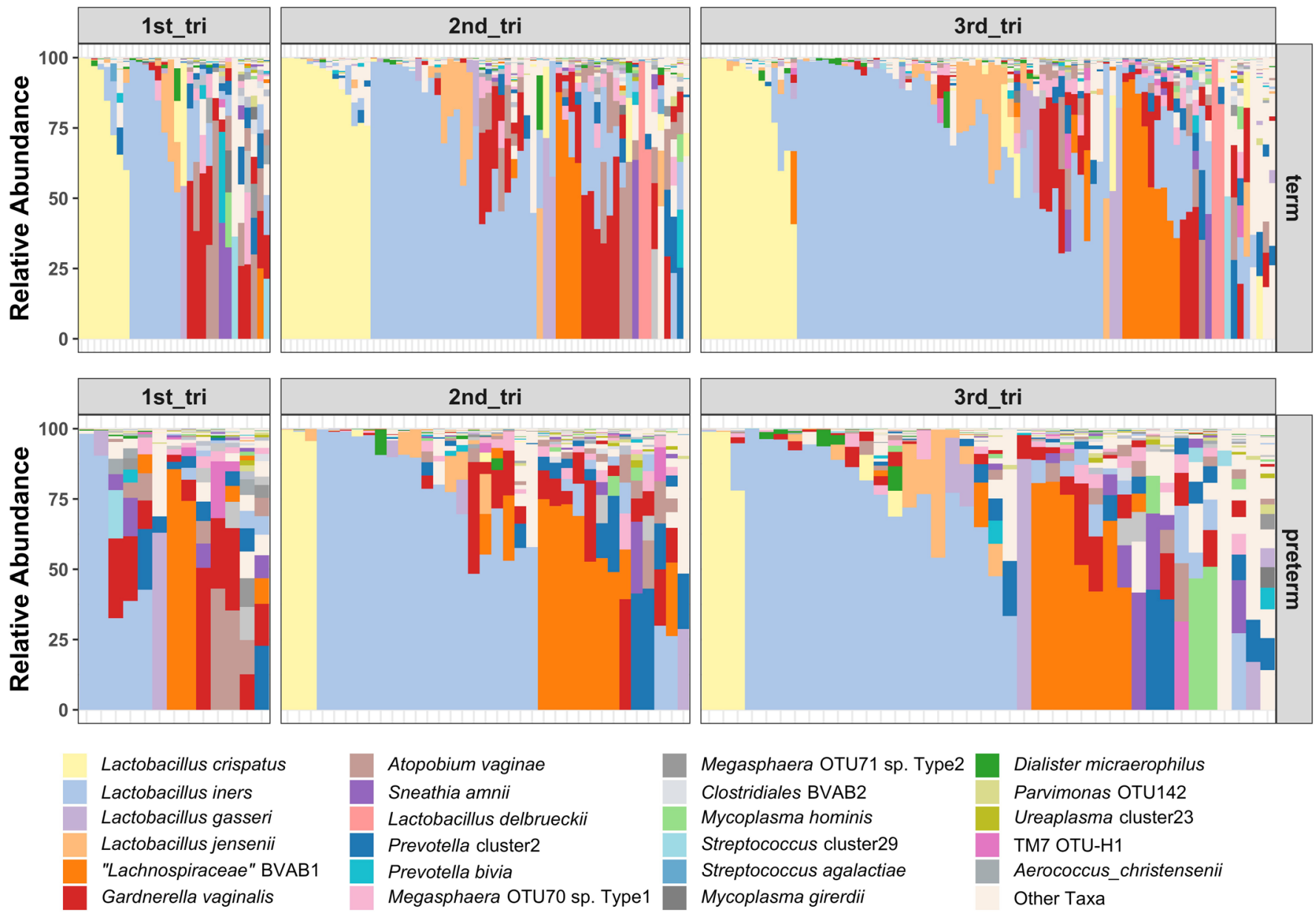

Megasphaera OTU71 sp. Type2 Clostridiales BVAB2 Mycoplasma hominis Streptococcus cluster29 Streptococcus agalactiae Mycoplasma girerdii
Dialister micraerophilus Parvimonas OTU142 Ureaplasma cluster23 TM7 OTU-H1 Aerococcus_christensenii Other Taxa

Extended Data Figure 1 | Species-level vaginal microbiome composition in women who experience TB or PTB. Stacked bar charts illustrating the vaginal microbiome profiles from $16 \mathrm{~S}$ rRNA surveys of one sample per trimester from each pregnancy. Samples are ordered according to decreasing relative abundance. Twenty-nine abundant taxa of interest are shown with all others pooled into 'Other'. 
a

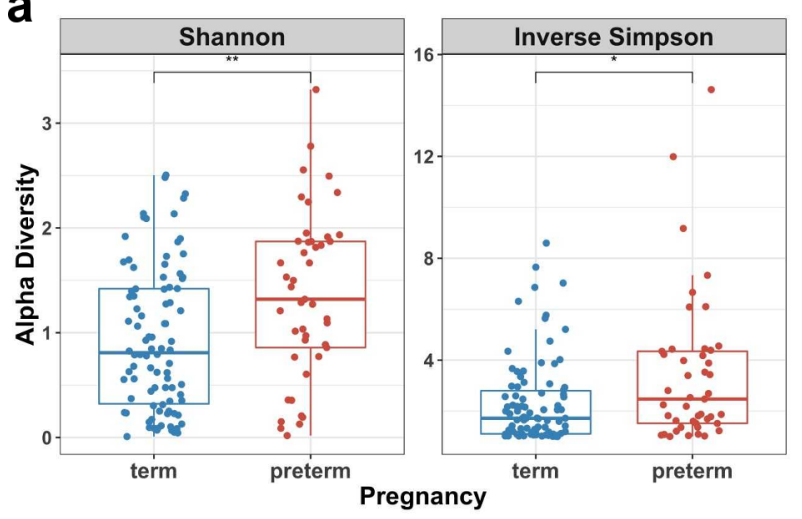

b
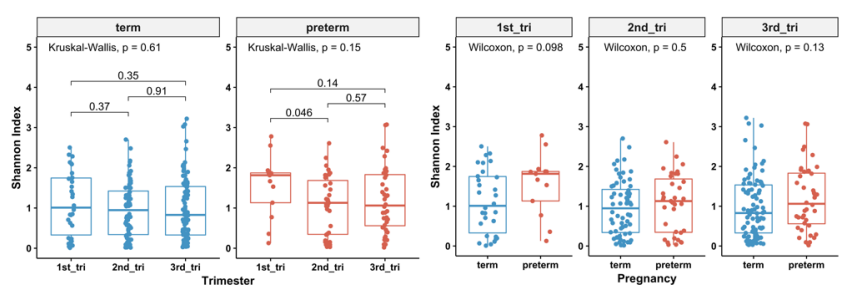

C

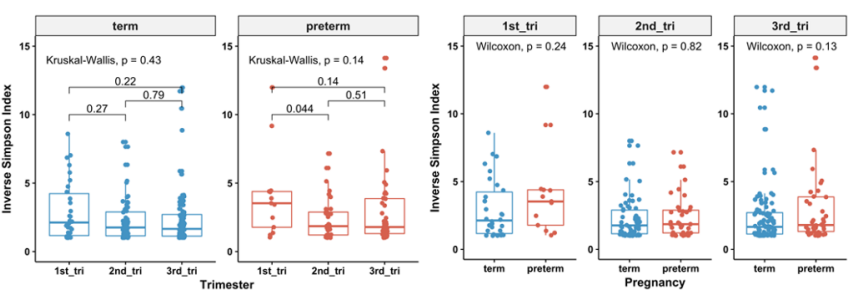

Extended Data Figure 2 | Alpha diversity measures for PTB and TB cohorts. a, Shannon index and inverse Simpson index for the cross-sectional cohort in Fig. 2 (PTB $n=45$, TB $n=90$ ) shows that alpha diversity is significantly higher $(P=0.0026, P=0.12$, respectively) in the preterm cohort using two-sided Wilcoxon's test followed by a Pvalue adjustment using Bonferroni's correction with $5 \%$ FDR. Boxes show median and interquartile range; whiskers extend from minimum to maximum values within each cohort. b,c Shannon index (b) and inverse Simpson index (c) diversity measures are shown with comparisons by trimester (TB first trimester: $n=30$; PTB first trimester: $n=13$; TB second trimester: $n=64$; PTB second trimester: $n=35$; TB third trimester: $n=90$; PTB third trimester: $n=40$ ). The Kruskal-Wallis two-sided test (analysis of variance) was used followed by post-hoc pairwise comparison with Bonferroni's correction and 5\% FDR. Comparison within trimesters was performed using two-sided Wilcoxon's test with Bonferroni's $P$ value adjustment. Boxes show median and interquartile range; whiskers extend from minimum to maximum values within each cohort. For the trimester comparisons, no tests were significant. 
a

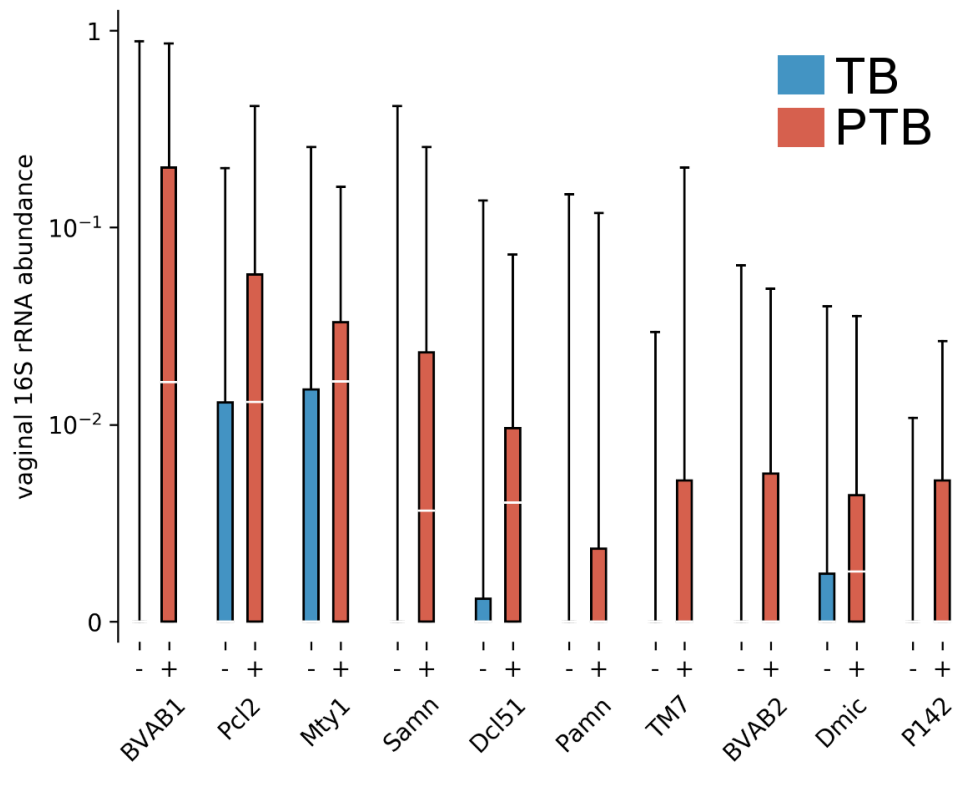

b

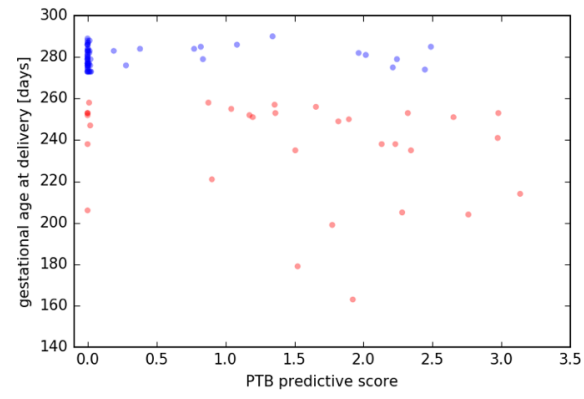

C

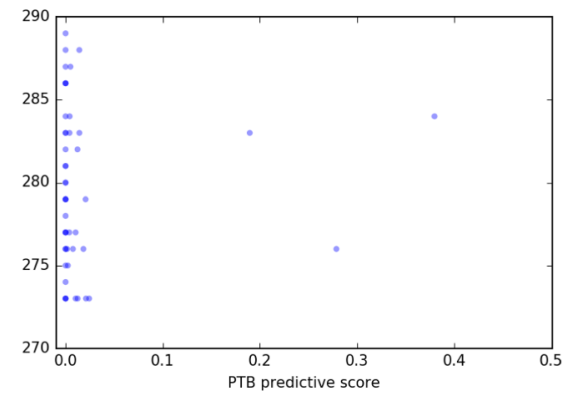

Extended Data Figure 3 | Taxa that significantly differ in PTB and TB cohorts. The distributions of proportional abundance of taxa greatly differ in PTB $(n=31)$ and TB $(n=59)$ cohorts; the earliest sample available for each subject within the first 24 weeks of pregnancy was used for each subject. Abundance values below 0.001 were rounded down to 0. The taxa are: BVAB1: Lachnospiraceae BVAB1, Pcl2: Prevotella cluster 2, Mty1: Megasphaera OTU70 type1, Samn: Sneathia amnii, TM7: TM7-H1, Dcl51: Dialister cluster 51, Pamn: Prevotella amnii, BVAB2: Clostridiales BVAB2, Dmic: Dialister micraerophilus and P142: Parvimonas OTU142. These 10 taxa have $P<0.05$ to support a significant difference in proportional abundance between PTB and TB cohorts using a Mann-Whitney U-test (two-sided) and the Benjamini-Hochberg correction procedure with an FDR of 5\%. Boxes show median and interquartile range; whiskers extend from minimum to maximum values within each cohort. $\mathbf{b}, \mathbf{c}$, Scatter plot of the PTB predictive score returned by the model (horizontal axis) plotted against gestational age at birth (vertical axis). Each point corresponds to a sample from a subject: red, PTB subjects $(n=31)$; blue, TB subjects $(n=59)$. c, Shows more detailed view of the region where most (48 of 59) of the TB samples are located. 


\section{PTB Metatranscriptomics}

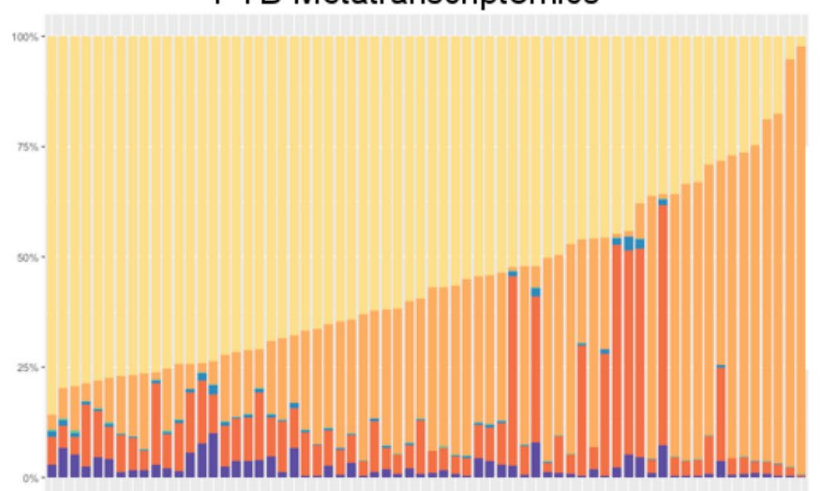

PTB Metagenomics

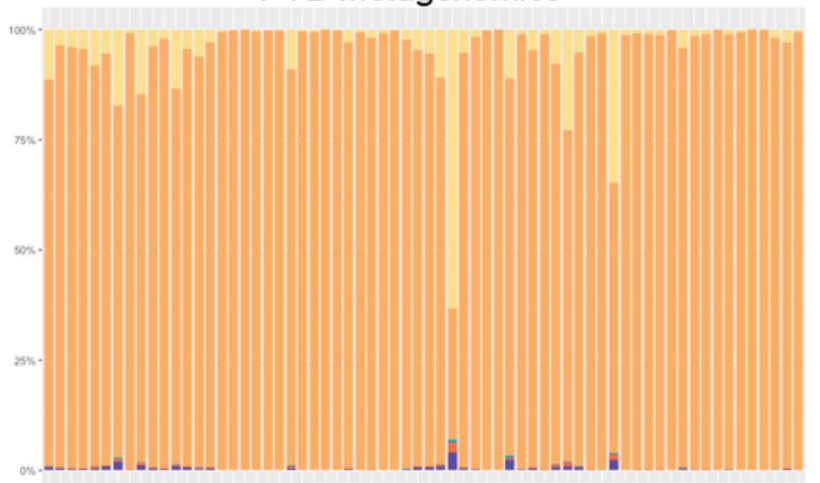

TB Metatranscriptomics

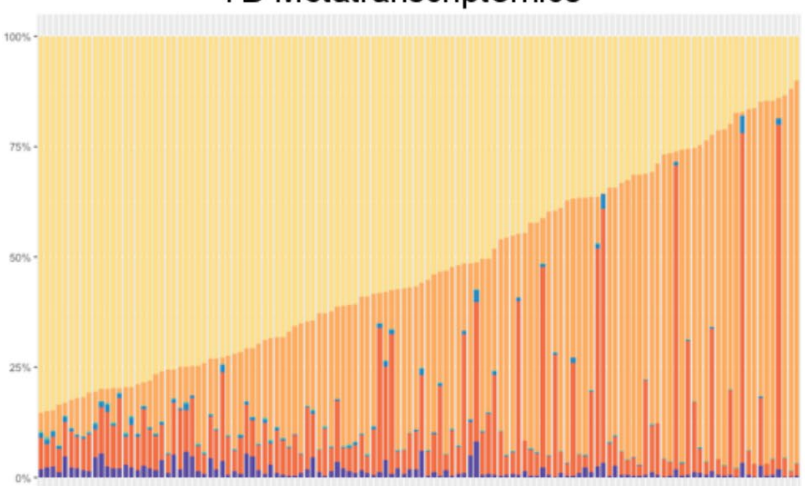

TB Metagenomics

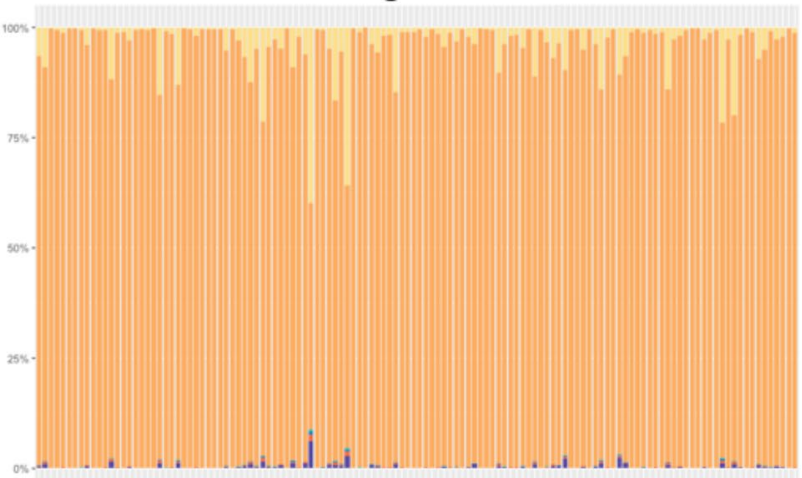

Assigned_Bacterial Unassigned_Ambiguity_Unassigned_NoFeatures

Assigned_Human_Unassigned_Chimera Uunassigned_Unmapped

Extended Data Figure 4 | Assignment of metatranscriptomics and metagenomics quality-filtered reads in PTB and TB cohorts. Non-human reads were mapped to a custom database corresponding to 56 bacterial taxa. Values are shown as the percentage abundance. 
2 16S rRNA surveys
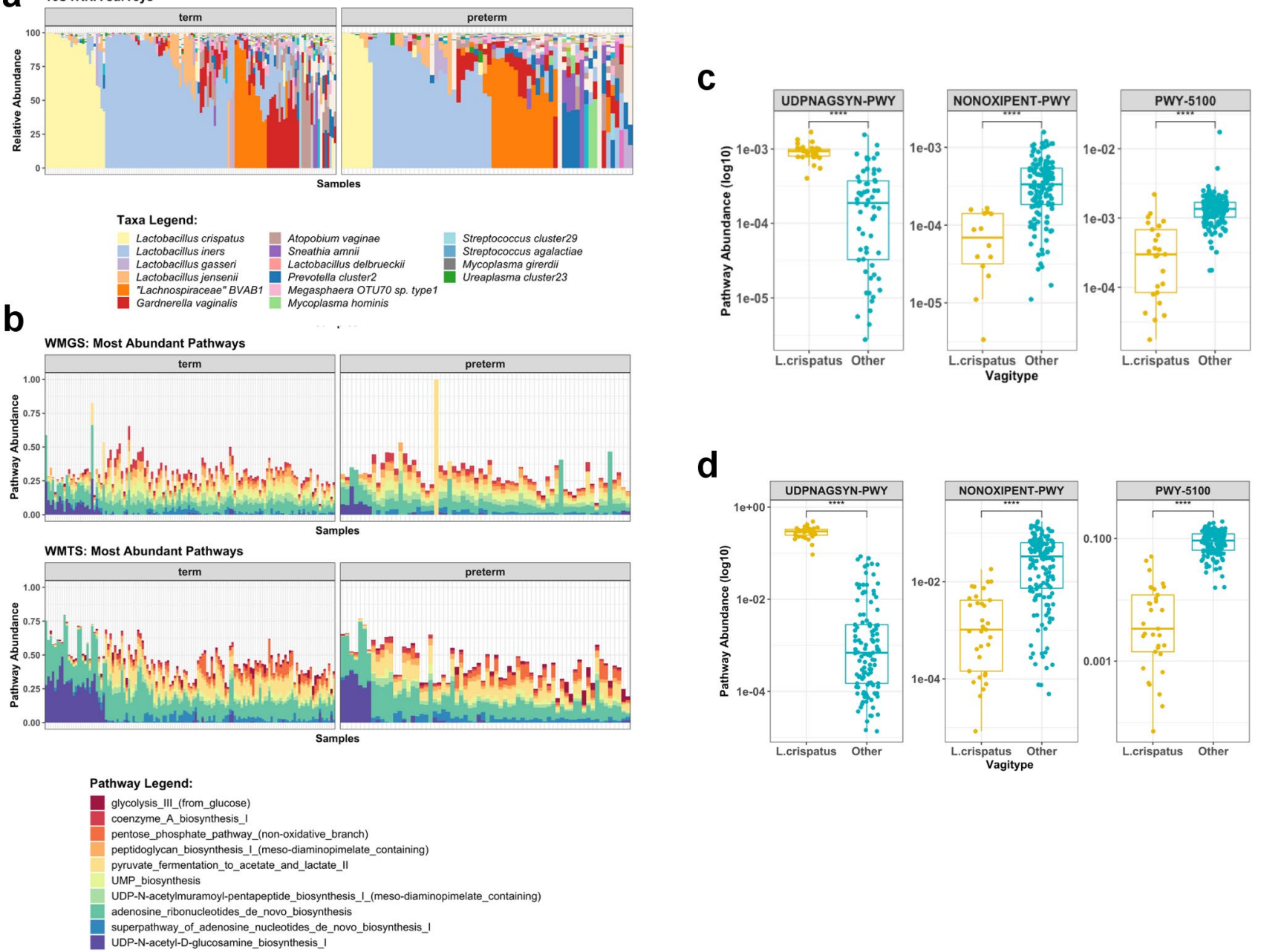

Extended Data Figure 5 | Metabolic pathway abundance by vagitype. a, Stacked barplots showing the vaginal microbial profiles from 165 rRNA surveys; b, the metabolic pathway abundances from paired MGS (top panel) and MTS (bottom panel) of vaginal samples collected during pregnancies of 90 women who delivered at term ( $\geq 39$ weeks) (left) and 45 women who delivered preterm ( $<37$ weeks) (right). Results show the top 10 metabolic pathways calculated by HUMAnN2. c,d, The presence of the UDP-N-acetyl-D-glucosamine biosynthesis pathway and non-oxidative branch of pentose phosphate pathway differ between $L$. crispatus and other vagitypes as measured by MGS data (c) (L. crispatus UDPNAGSYN: $n=32$, Other UDPNAGSYN: $n=59 ;$. crispatus NONOXYPENT-PWY: $n=14$, Other NONOXYPENT-PWY: $n=138$; L. crispatus PWY-5100: $n=25$,Other PWY-5100: $n=156$ ) and MTS data (d) (L. crispatus UDPNAGSYN: $n=33$, Other UDPNAGSYN: $n=117$; L. crispatus NONOXYPENT-PWY: $n=33$, Other NONOXYPENT-PWY: $n=157 ;$ L. crispatus PWY-5100: $n=33$,Other PWY-5100: $n=159)$. Two-sided Wilcoxon's $P$ test $(P<0.05)$ with Bonferroni's adjustment at a $5 \%$ FDR threshold was used. Boxes show median and interquartile range; whiskers extend from minimum to maximum values within each cohort. 

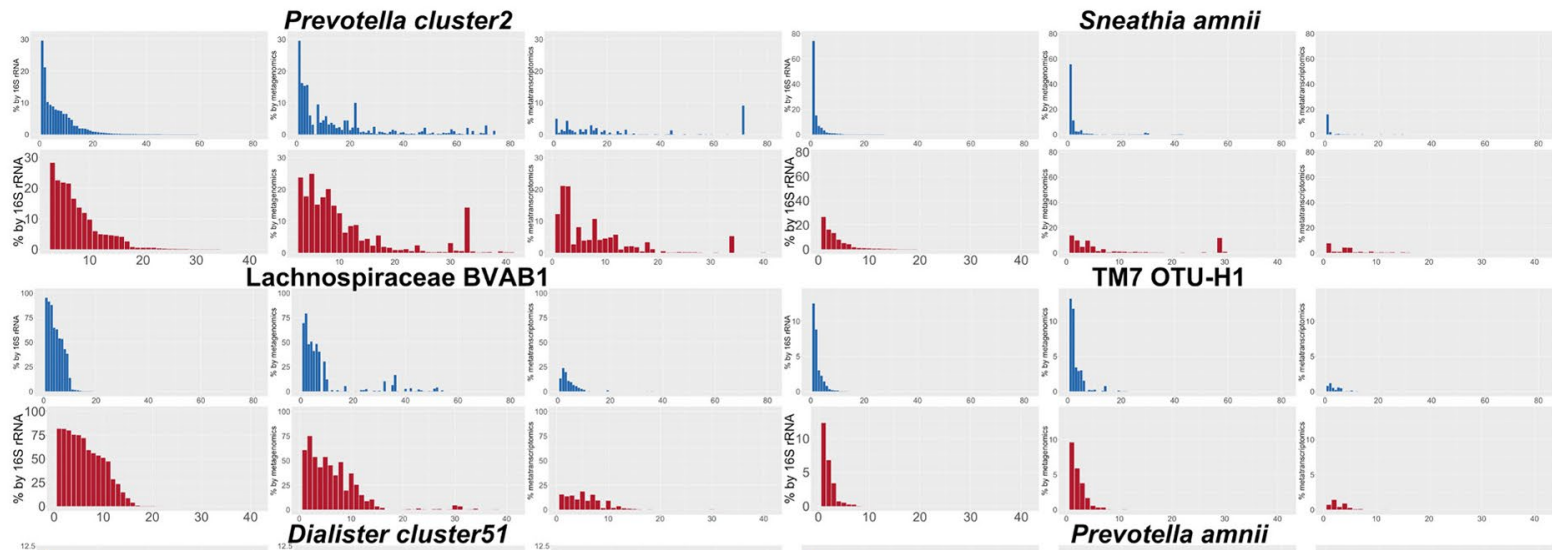

TM7 OTU-H1
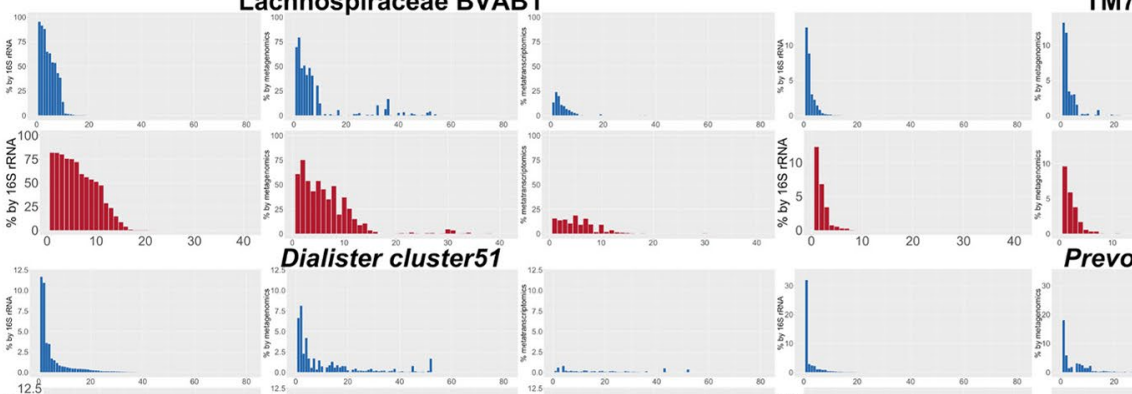

Inhlin..
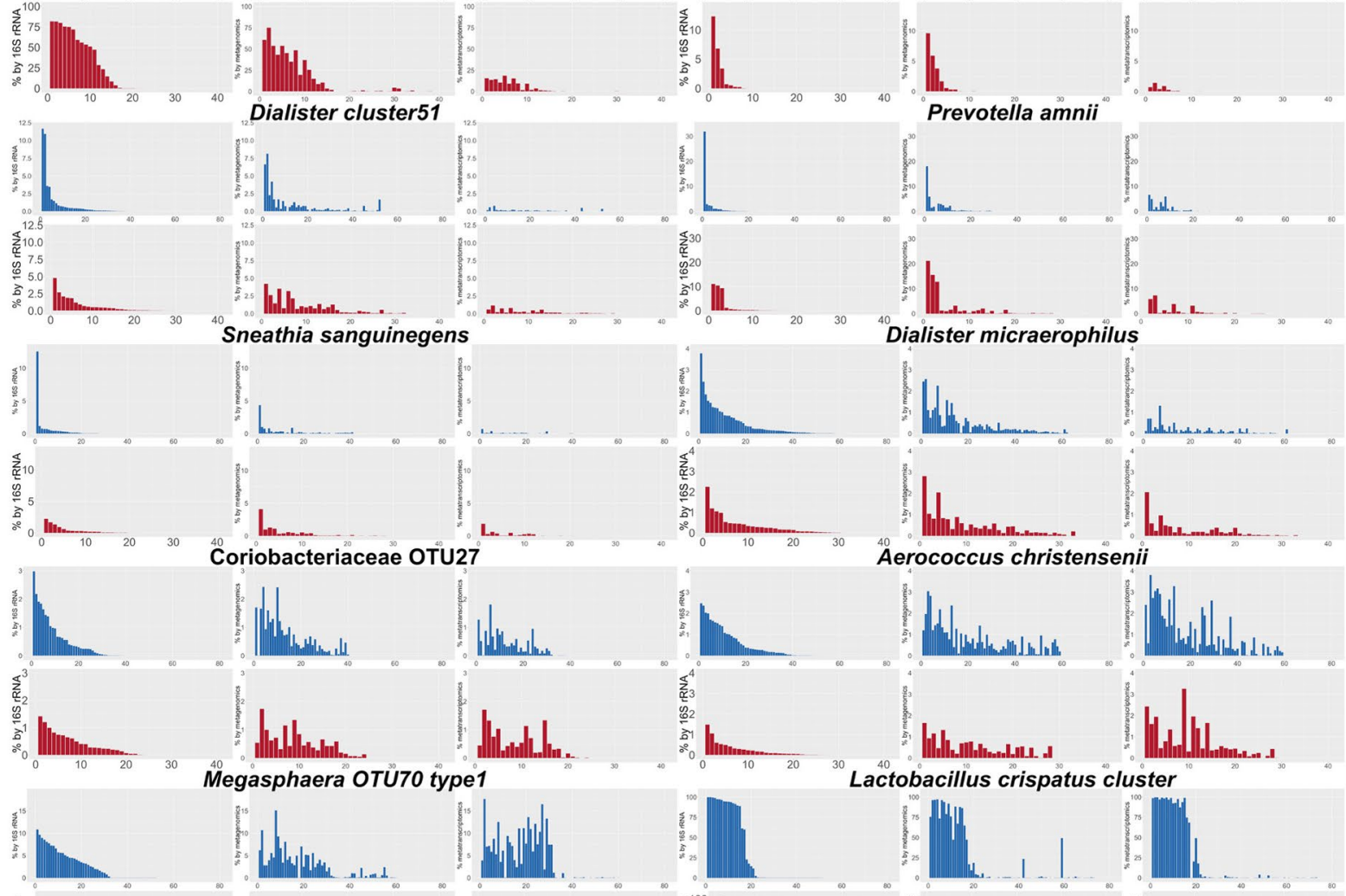

Megasphaera OTU70 type1
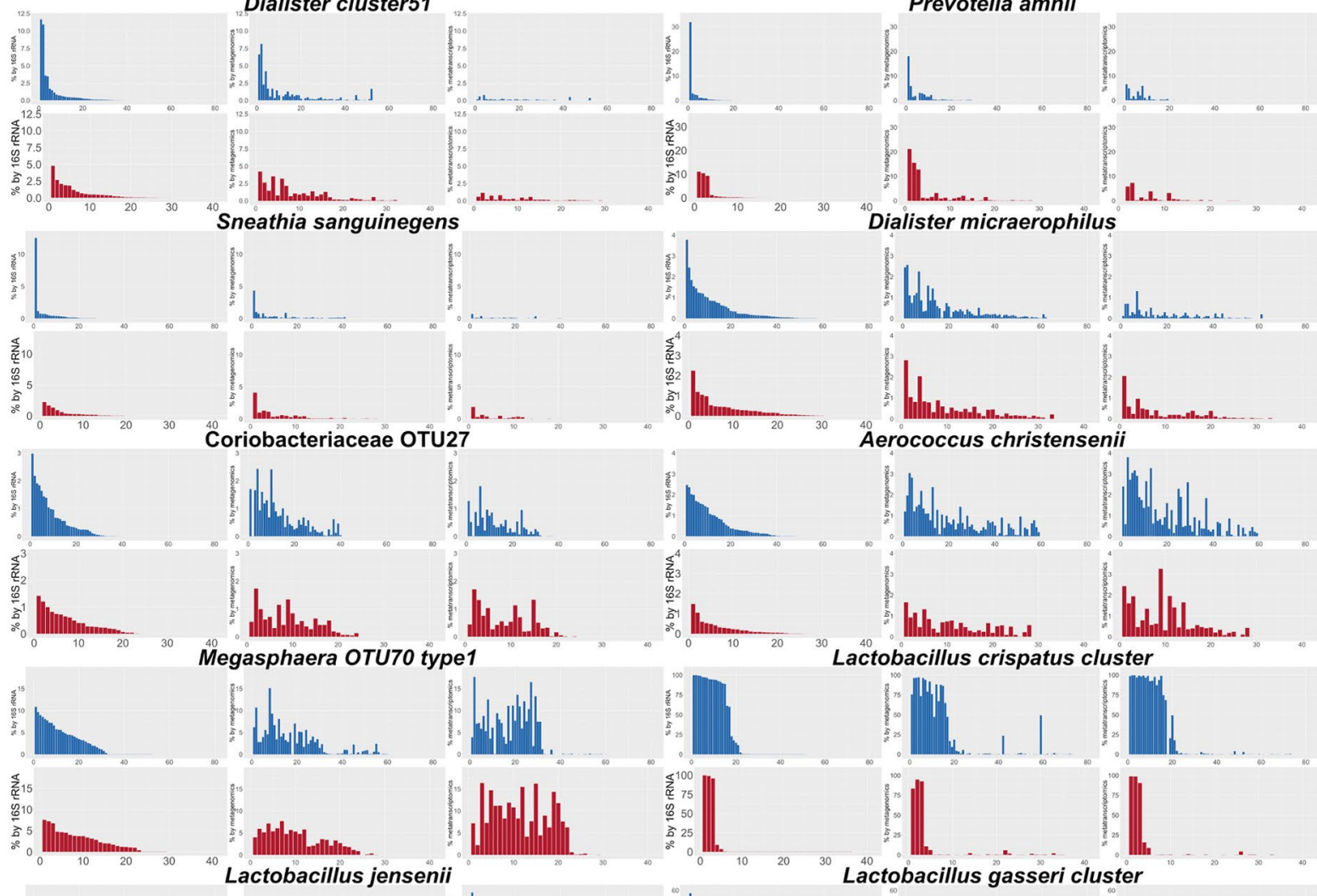

Aerococcus christensenii
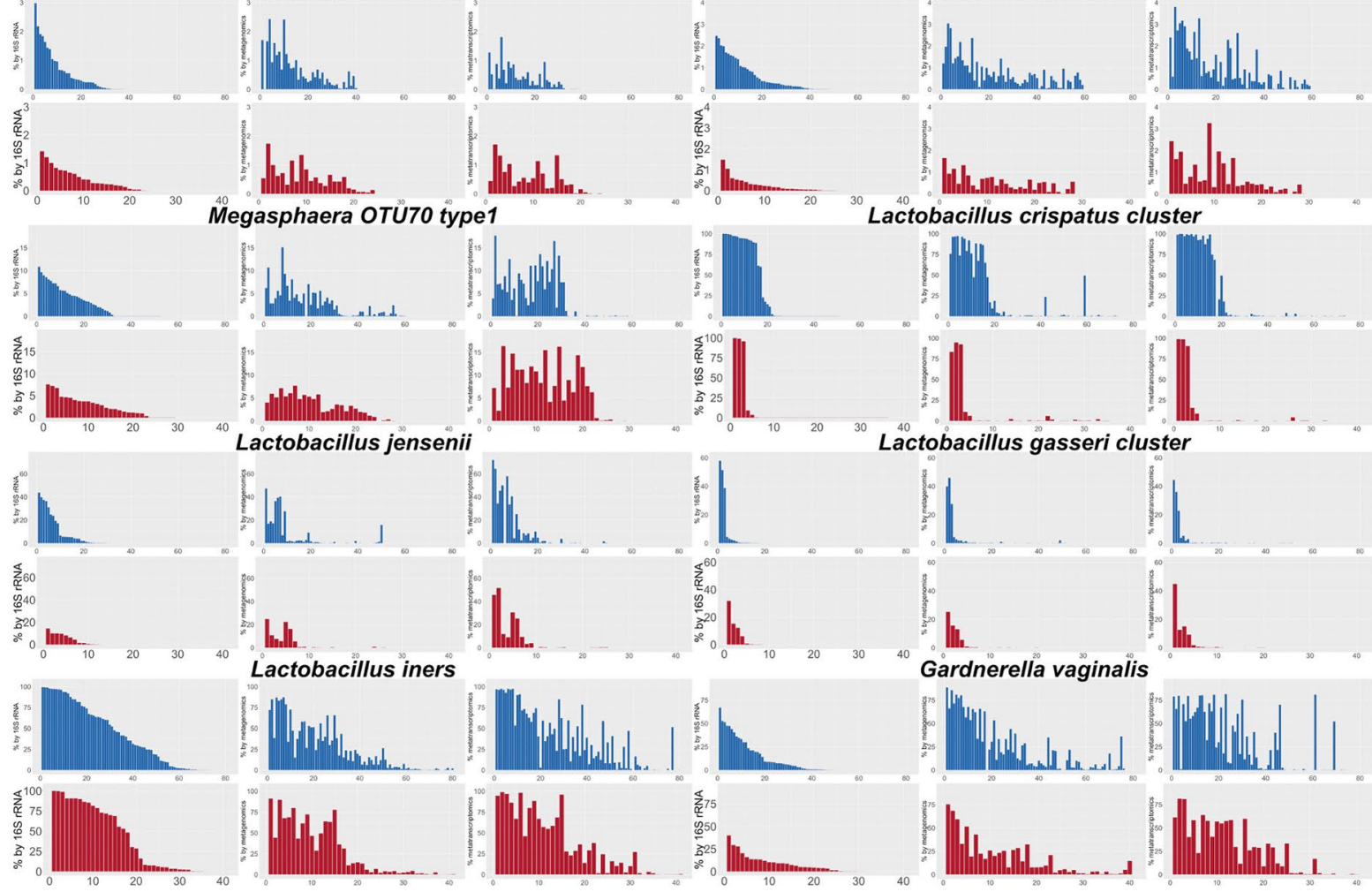

Extended Data Figure 6 | Comparison of the proportional abundance of taxa by 16S rRNA (left), metagenomics (middle) and metatranscriptomics (right) in term (blue) and preterm (red) cohorts. Proportional abundance is shown relative to the 56 bacterial taxa that were measured across all three assays. The $16 \mathrm{~S}$ rRNA, metagenomics and metatranscriptomics measures from PTB $(n=41)$ and TB $(n=81)$ were from a single time point per participant. The $y$ axis is scaled based on the maximum proportional abundance for the taxon. 
a
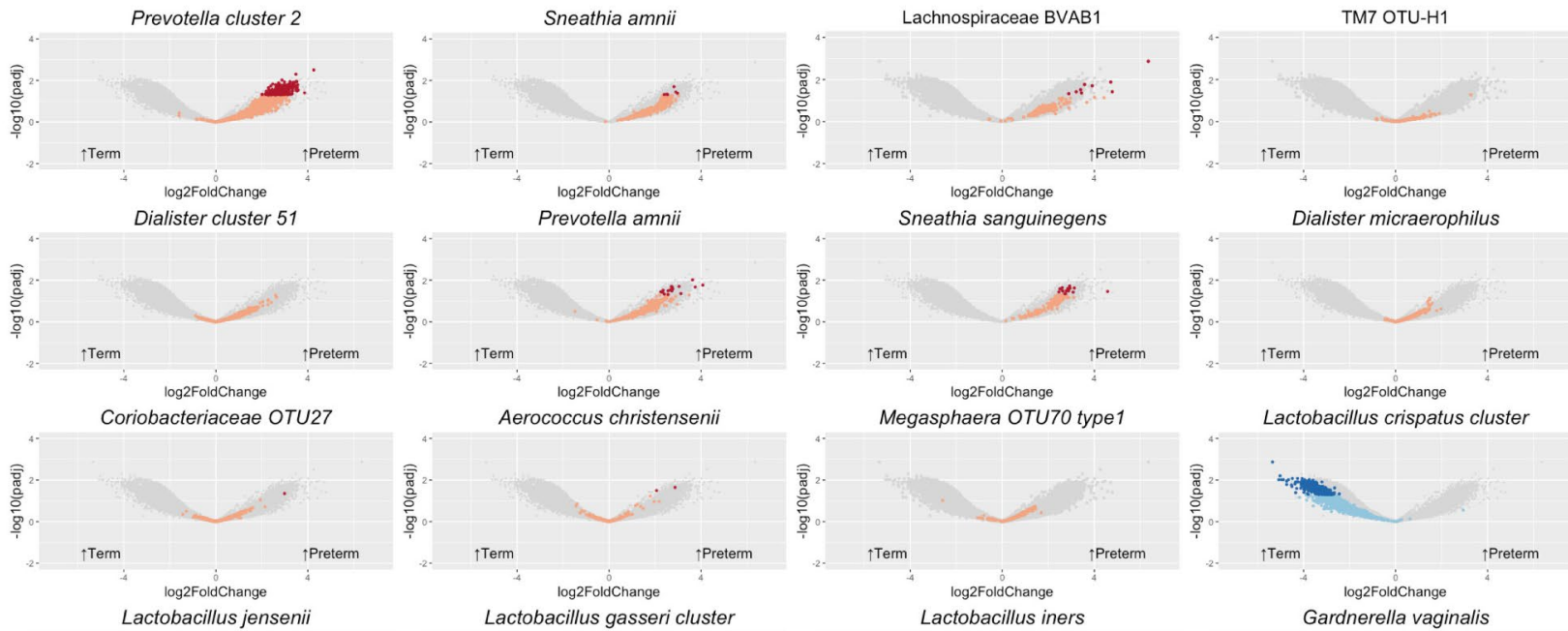

Lactobacillus crispatus cluster
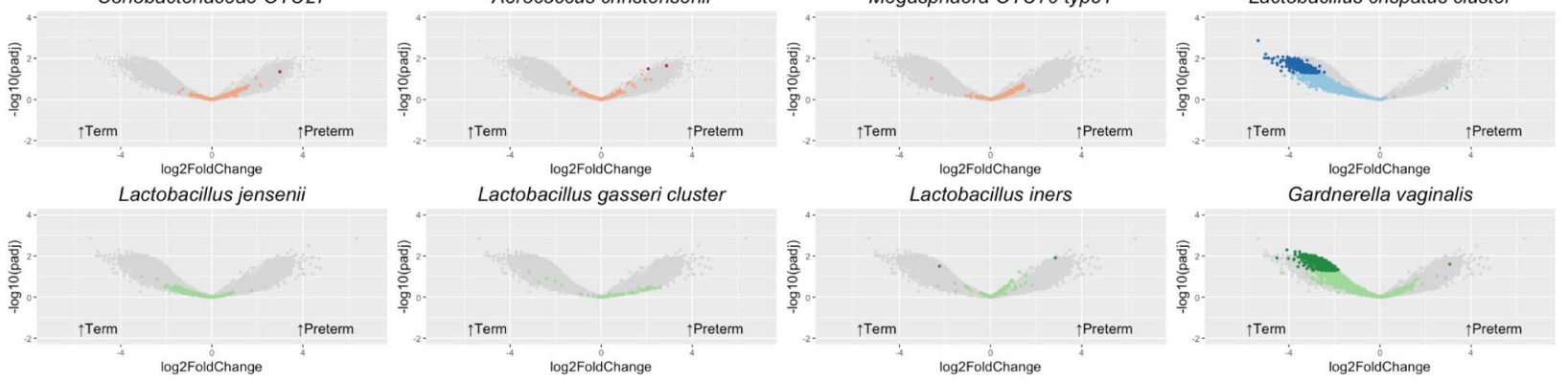

b

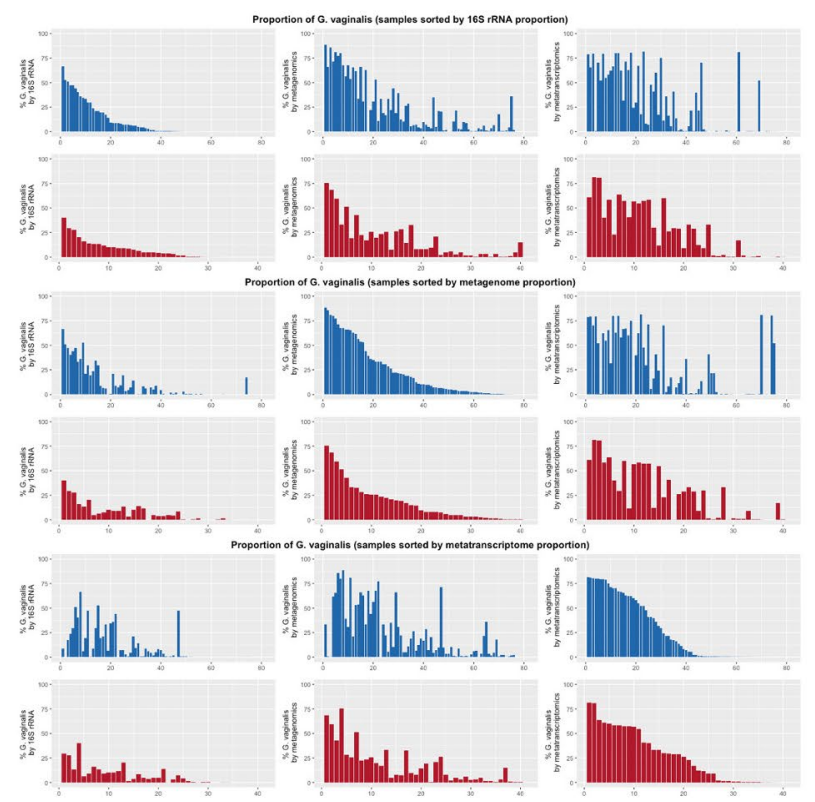

C

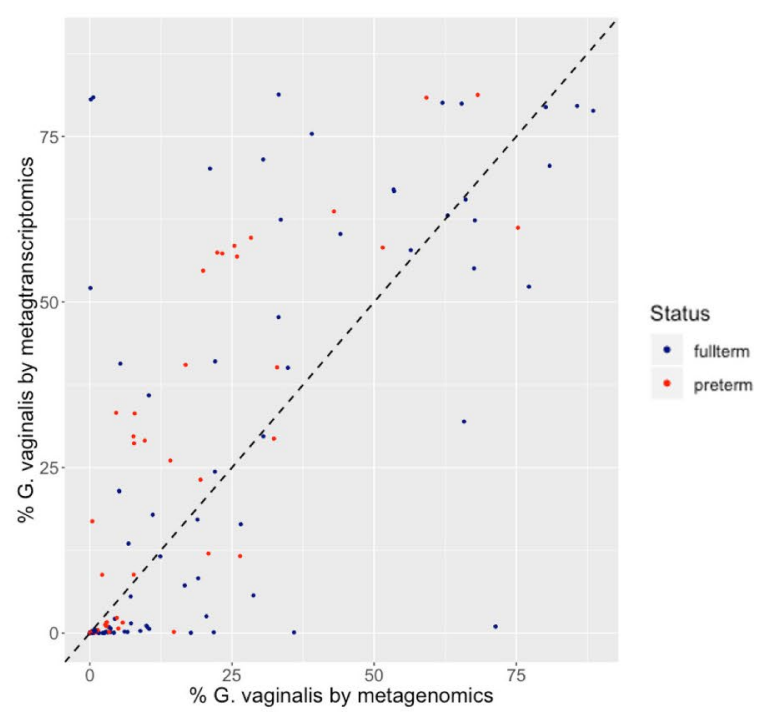

Extended Data Figure 7 | Genes differing in metagenomic and metatranscriptomic data between term and preterm cohorts. a, Volcano plot depicting genes that significantly differ between term $(n=41)$ and preterm $(n=81)$ cohorts in metagenomics data. Genes mapping to PTB-associated taxa, the $L$. crispatus cluster and four other common taxa that significantly differ at $P_{\text {adj }}<0.05$ (two-sided) with FDR correction are shown in dark red, dark blue and dark green, respectively. b. Comparison of proportional abundance of G. vaginalis in preterm (red) and term (blue) vaginal samples as assayed by $16 \mathrm{~S}$ rRNA (left), metagenomics (middle) and metatranscriptomics (right), sorted by $16 \mathrm{~S}$ rRNA (top), metagenomics (middle) and metatranscriptomics (bottom) data. $\mathbf{c}$, Scatter plot of the abundance of $G$. vaginalis by metagenomics ( $x$ axis) and abundance of $G$. vaginalis by metatranscriptomics ( $y$ axis) in pregnant women who delivered term (blue) or preterm (red). Two-sided Wilcoxon's test of the ratio of the log-transformed proportions (log (metatranscriptomics $G$. vaginalis proportion)/log(G. vaginalis metagenomics proportion)) showed a significant difference between term and preterm groups $(P=0.01069)$. 
a

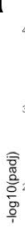

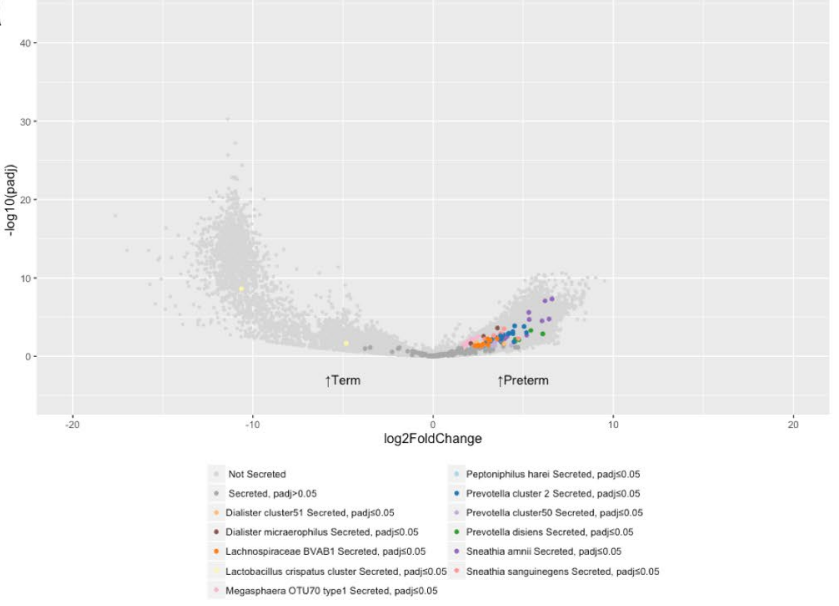

b

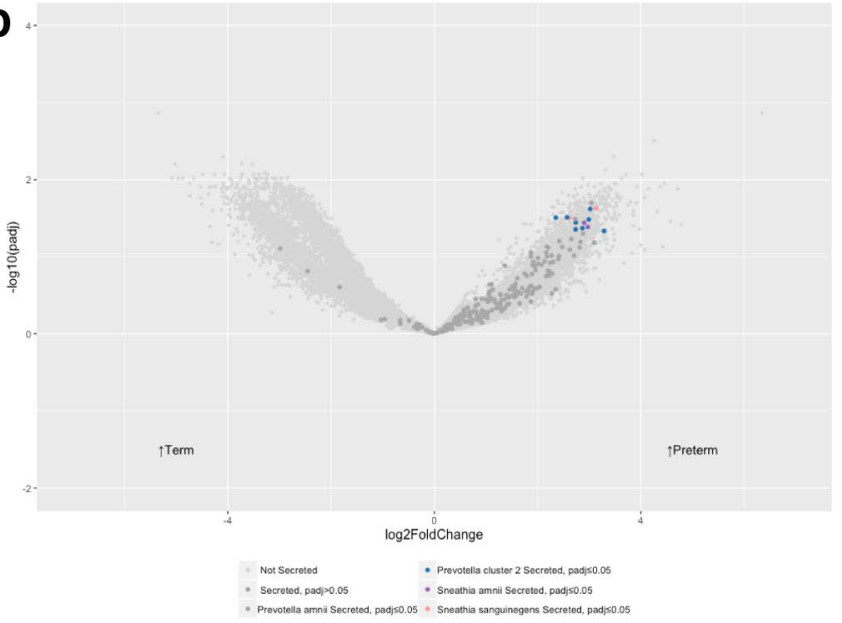

Extended Data Figure 8 | Genes predicted to encode secreted proteins or to be involved in bacterial secretion systems in metatranscriptomics and metagenomics datasets. Genes predicted to encode secreted proteins or to be involved in bacterial secretion systems that have very different transcript levels (a) or very different levels in metagenomics data $(\mathbf{b})$ in term $(n=81)$ and preterm $(n=41)$ cohorts. Analysis was performed by mapping metatranscriptomics (a) or metagenomics (b) reads to a customized database of genomes representing 56 taxa. Comparative analysis was performed with DESeq2 using a global scaling approach. All genes with an FDR-adjusted $P_{\text {adj }}<0.05$ (two-sided test), which are also predicted to be involved in bacterial secretion as identified by MacSyFinder, are shown and colored by taxon. 
a
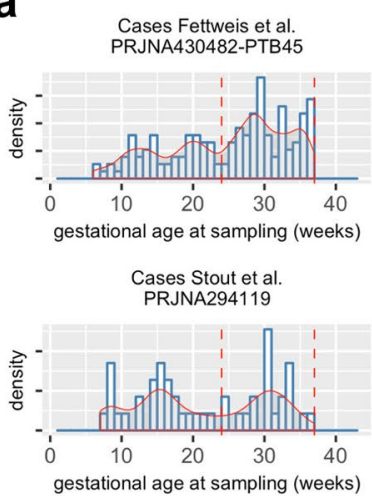

Cases Romero et al.

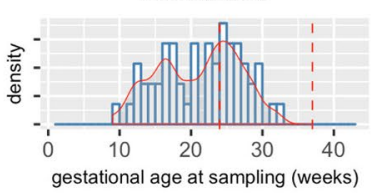

Cases Callahan et al.

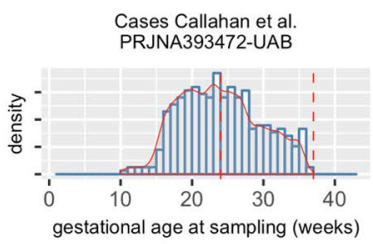

C

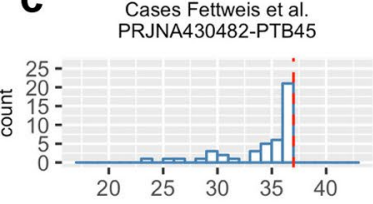

gestational age at delivery (weeks)

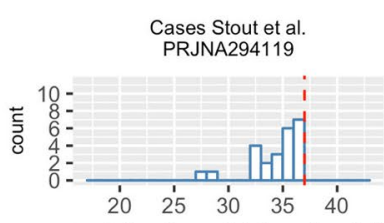

gestational age at delivery (weeks)

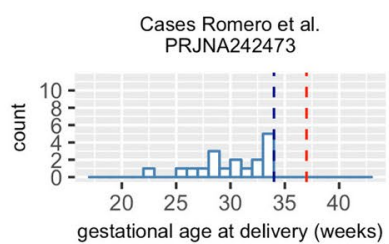

gestational age at delivery (weeks)

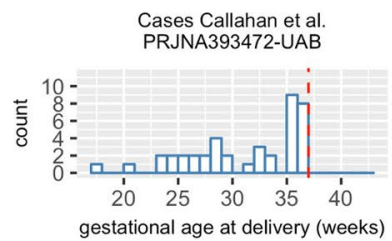

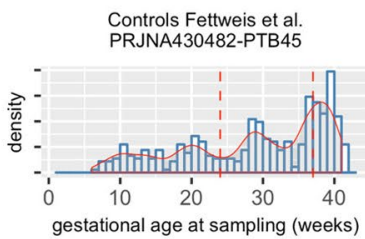
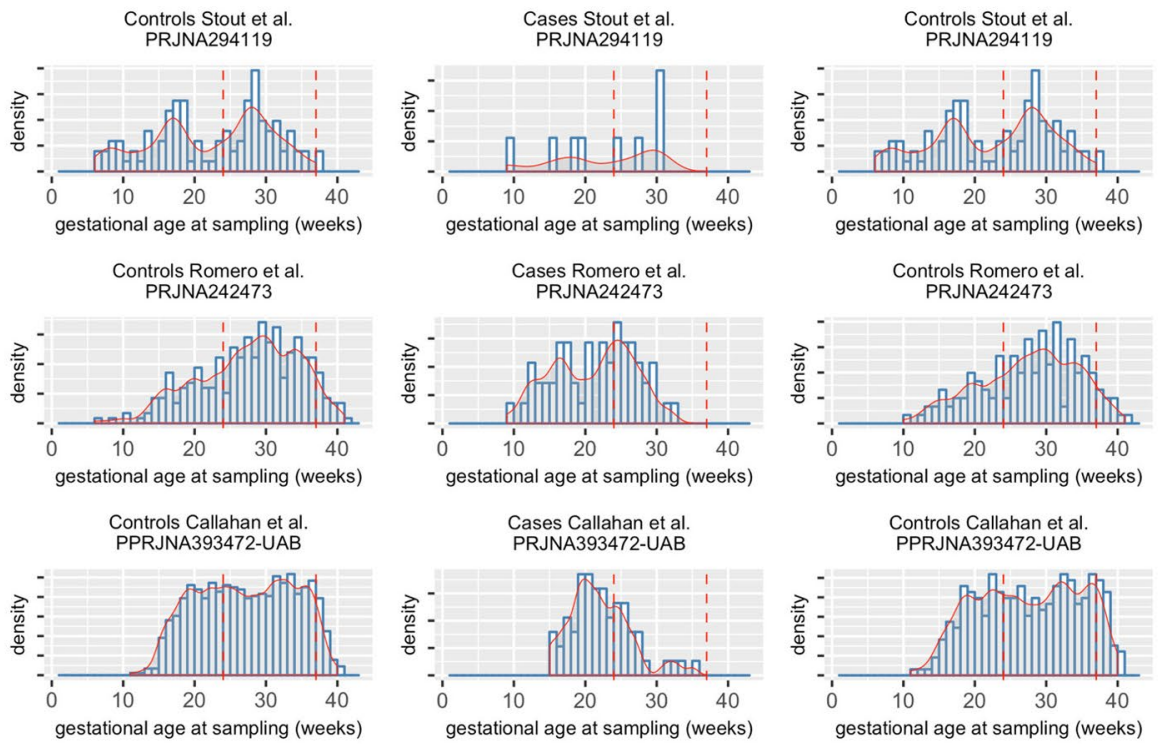
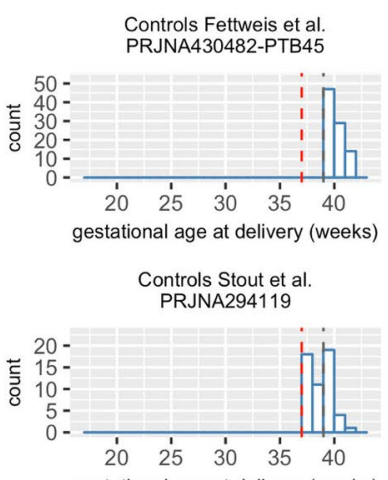

gestational age at delivery (weeks)
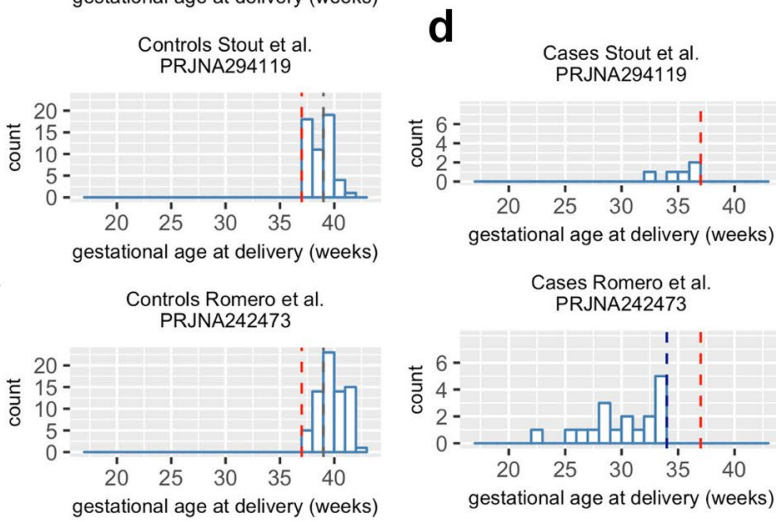

Cases Romero et al. PRJNA242473

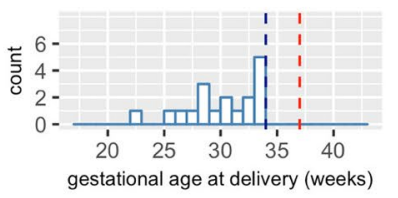
Cases Callahan et al.
PRJNA393472-UAB
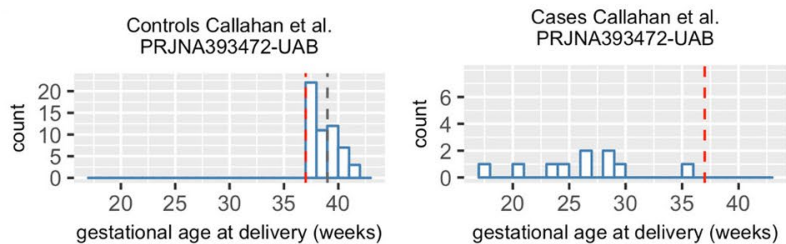

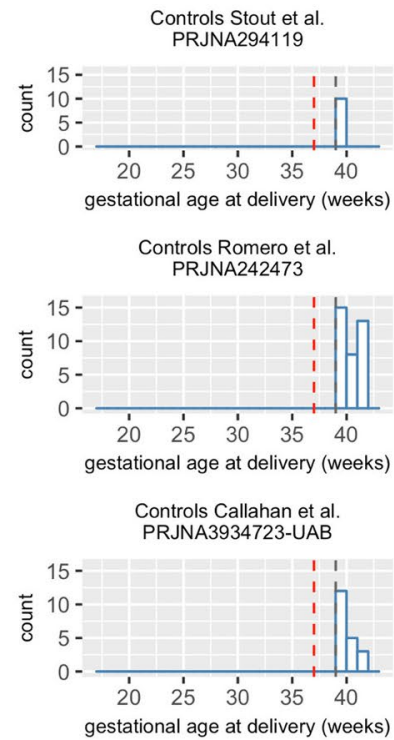

Extended Data Figure 9 | Distribution of sampling and gestational age at delivery across studies of the vaginal microbiome and PTB. The panels show the distribution of the gestational age at sampling (a) and gestational age at delivery (c) in preterm cases (left) and term controls (right) across four studies: the present study (top), the Stout et al. study, the Romero et al. study and the Callahan et al. UAB cohort (bottom), as originally published, and in b,d for the reanalyzed cohorts. 
a

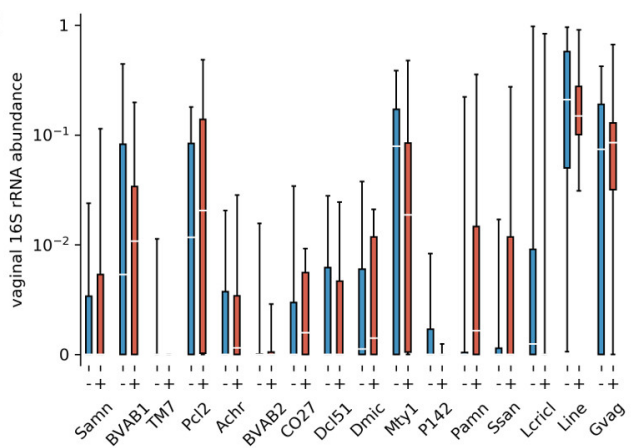

C

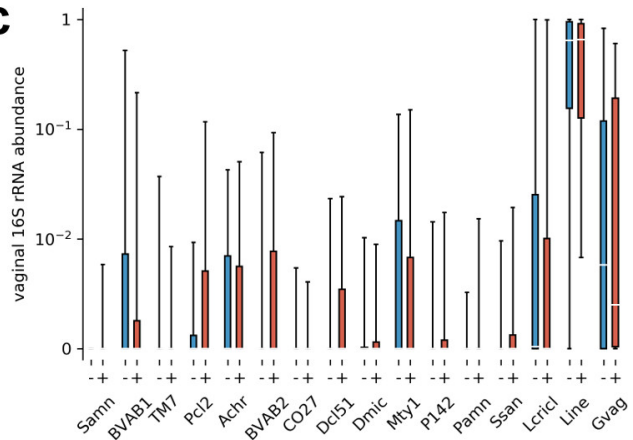

b

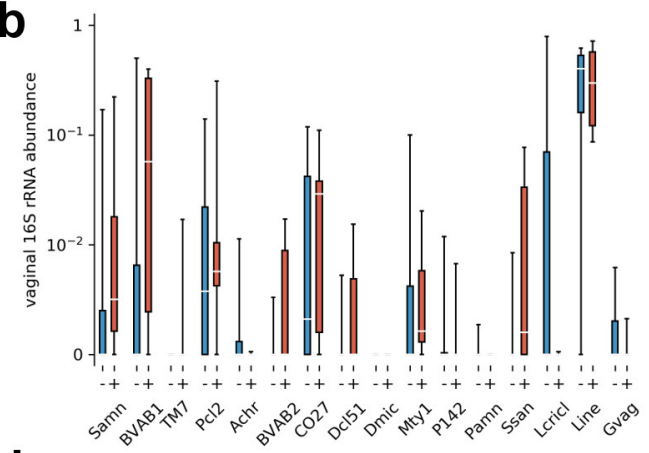

d

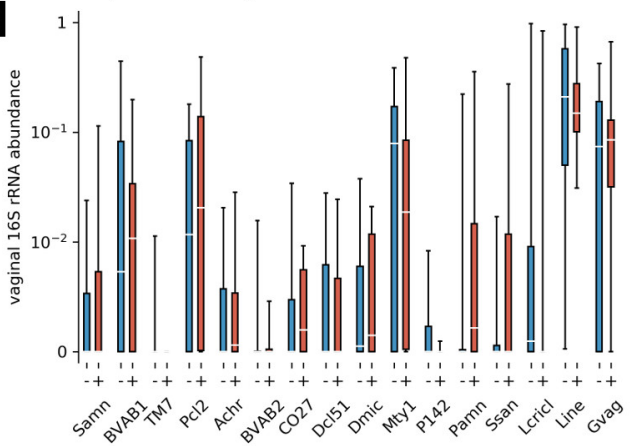

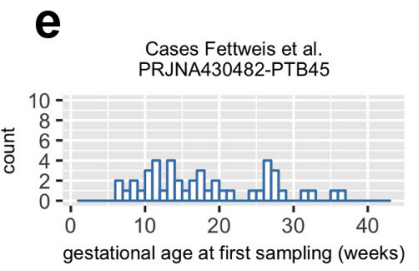

Cases Stout et al

PRJNA294119

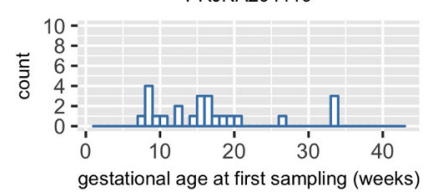

Cases Romero et al.
PRJNA242473

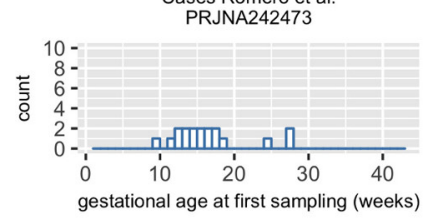

Cases Callahan et al.

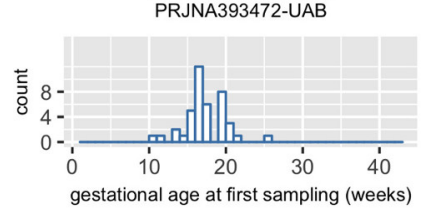

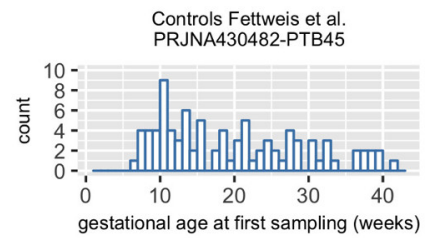

Controls Stout et al.

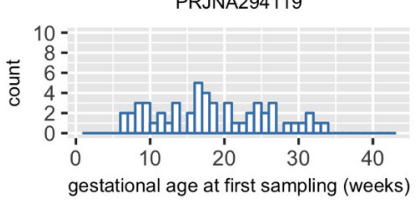

f
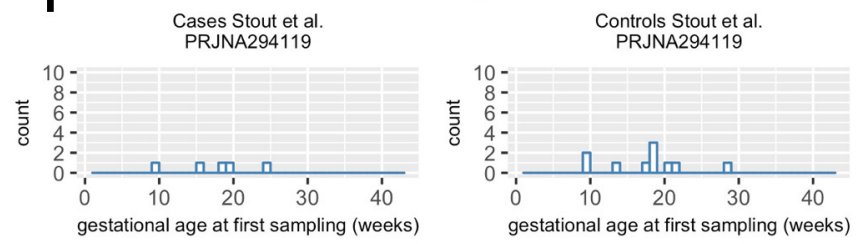

Controls Romero et al.
PRJNA242473
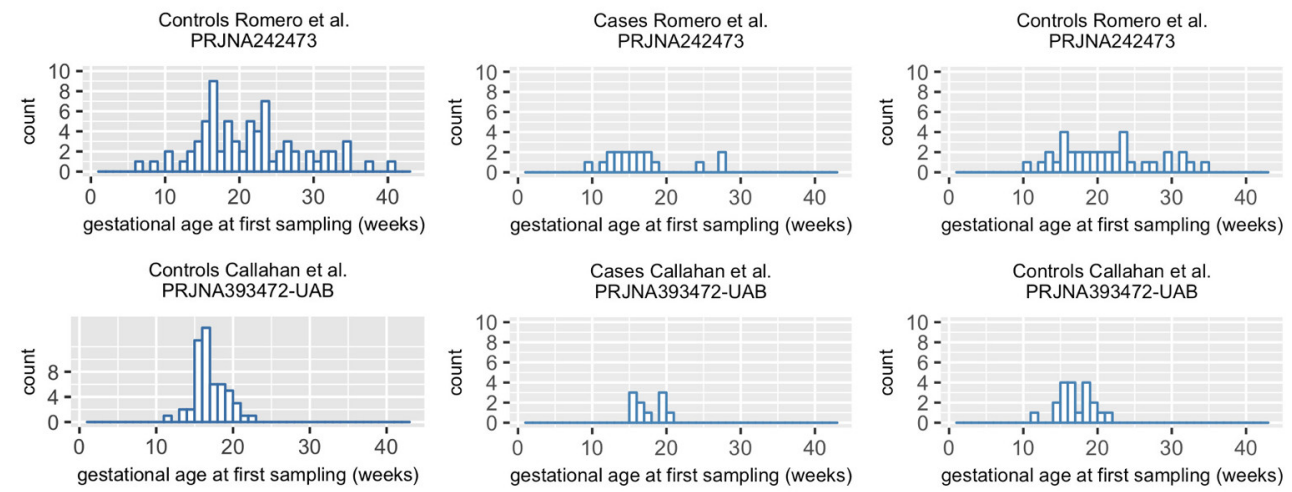

Extended Data Figure 10 | Proportional abundance shown as a log scale of candidate taxa identified in the present study across four PTB cohorts. a-d, Abundance values below 0.001 were rounded down to 0 . Boxes show median and interquartile range; whiskers extend from minimum to maximum values within each cohort for the present study $(P T B n=45, T B n=90)(\mathbf{a})$, the Stout et al. replication cohort $(P T B n=5, T B n=10)(\mathbf{b})$, the Romero et al. cohort (PTB $n=18, T B n=36$ ) (c) and the Callahan et al. UAB replication cohort (PTB $n=10, T B n=20)$ (d). The Mann-Whitney U-test (two-sided) for difference in proportional abundance between the PTB and TB cohorts, corrected using the Benjamini-Hochberg procedure with an FDR of 5\% did not show statistical significance for these taxa in the cohorts shown in $\mathbf{b}$-d. The earliest sample available for each subject was used (f). Earliest samples for the original four cohorts are shown in $\mathbf{e}$. 


\section{natureresearch}

Corresponding author(s): Gregory Buck

Last updated by author(s): Mar 18, 2019

\section{Reporting Summary}

Nature Research wishes to improve the reproducibility of the work that we publish. This form provides structure for consistency and transparency in reporting. For further information on Nature Research policies, see Authors \& Referees and the Editorial Policy Checklist.

\section{Statistics}

For all statistical analyses, confirm that the following items are present in the figure legend, table legend, main text, or Methods section.

n/a Confirmed

\ The exact sample size $(n)$ for each experimental group/condition, given as a discrete number and unit of measurement

$\square$ A statement on whether measurements were taken from distinct samples or whether the same sample was measured repeatedly

The statistical test(s) used AND whether they are one- or two-sided

Only common tests should be described solely by name; describe more complex techniques in the Methods section.

$\square$ A description of all covariates tested

$\square \bigotimes$ A description of any assumptions or corrections, such as tests of normality and adjustment for multiple comparisons

$\checkmark$ A full description of the statistical parameters including central tendency (e.g. means) or other basic estimates (e.g. regression coefficient)

$\triangle$ AND variation (e.g. standard deviation) or associated estimates of uncertainty (e.g. confidence intervals)

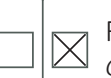

For null hypothesis testing, the test statistic (e.g. $F, t, r$ ) with confidence intervals, effect sizes, degrees of freedom and $P$ value noted

Give $P$ values as exact values whenever suitable.

$\square$ For Bayesian analysis, information on the choice of priors and Markov chain Monte Carlo settings

$\square$ For hierarchical and complex designs, identification of the appropriate level for tests and full reporting of outcomes

$\square$ Estimates of effect sizes (e.g. Cohen's $d$, Pearson's $r$ ), indicating how they were calculated

Our web collection on statistics for biologists contains articles on many of the points above.

\section{Software and code}

Policy information about availability of computer code

Data collection

Custom code will be available at https://github.com/Vaginal-Microbiome-Consortium/PTB prior to publication. Open source software is described in the test.

Data analysis

MeFiT for preprocessing of short read pair-end sequences, meep for quality filtering of paired-end sequences, QIIME for assignment of reads to Operational Taxonomic Units, Greengenes for $16 \mathrm{~S}$ rRNA refrence sequences, UCHIME to screen for chimeric sequences, STIRRUPS using USEARCH for species-level classification of reads, bcl2fastq conversion software from Illumina for data demultiplexing, Adapter Removal tool v 2.1.3 to trim adapters, meeptools for quality trimming of reads, BWA for alignment of reads to reference sequences, MetaPhIAn2 for analysis of metagenomic and metatranscriptomic sequence, ASGARD, HUMAnN2 and ShortBRED for assignment of genes to pathways, BLAST for alignment of sequences, BBMap for normalizing of reads, SPAdes ver 3.8.0 for assembly of reads, Bowtie2 for alignment of reads to scaffolds, MyCC for clustering metagenomic contigs into specific taxonomic units, Newbler Assembler v 2.8 for assembly of reads, Prokka for gene annotation, HGAP for assembly of metagenomes from Pac Bio long read data, MacSysFinder was used to identify genes involved in bacterial secretion systems, FeatureCounts was used to count paired-end reads where both ends mapped to non-ribosomal genes, DESeq2 was used to compare term and preterm cohorts using an organismindependent global-scaling approach, MultiQuant software was used for analysis of lipidomic spectral data, various version of R were used for statistical profiling, REBACCA statistical tool to mitigate effects of relative constrain, Gephi for visualization of bacterial correlations, and a variety of custom scripts (deposited at GitHub) were used to generate the figures in the manuscript.

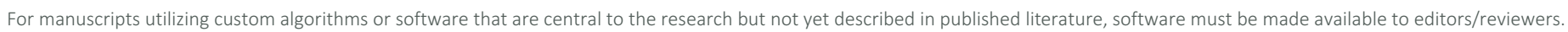
We strongly encourage code deposition in a community repository (e.g. GitHub). See the Nature Research guidelines for submitting code \& software for further information. 
Policy information about availability of data

All manuscripts must include a data availability statement. This statement should provide the following information, where applicable:

- Accession codes, unique identifiers, or web links for publicly available datasets

- A list of figures that have associated raw data

- A description of any restrictions on data availability

Open-access data including raw $16 \mathrm{~S}$ rRNA sequences, cytokine data and limited metadata are available at the HMP DACC (https://portal.hmpdacc.org/). Controlledaccess data including raw metagenomic sequences, raw metatranscriptomic sequences and metadata for all subjects analyzed in this study are available at NCBI's controlled-access dbGaP (study number: 20280; accession ID: phs001523.v1.p1) and the Sequence Read Archive (SRA) under BioProject ID PRJNA326441. The genomes of TM7-H1(CP026537) and BVAB1 (PQVO000000) have been submitted to GenBank. Access to additional fields can be requested through the RAMS Registry (https://ramsregistry.vcu.edu). Additional project information is available at the project's website (http://vmc.vcu.edu/momspi).

\section{Field-specific reporting}

Please select the one below that is the best fit for your research. If you are not sure, read the appropriate sections before making your selection.

$\bigotimes$ Life sciences

Behavioural \& social sciences

Ecological, evolutionary \& environmental sciences

For a reference copy of the document with all sections, see nature.com/documents/nr-reporting-summary-flat.pdf

\section{Life sciences study design}

All studies must disclose on these points even when the disclosure is negative.

Sample size In our initial power analysis (grant submission) we estimated with a $\Delta \geq 0.5$, we would have power $>98 \%$ with only 50 samples per group to identify differences between term and preterm samples. These were projected to include 'all' preterm births, including over $50 \%$ due to multiparous pregnancies and other medical reasons. In this project, we selected only 'spontaneous' preterm births, which have no obvious medical etiology. From the participants in the project, we were able to identify only 47 women who met all of our inclusion/exclusion criteria for spontaneous preterm births. These participants would have a higher delta than 'all' women who experience preterm birth. Moreover, several recent publications have used as low as 9 or fewer spontaneous preterm births, and no earlier publication had as many as 47 . Thus, we believed this was a sufficient number to begin with. We were able to match these 47 with 94 term births to increase the statistical power of our analysis. In the end, we eliminated 2 preterm births (and their 4 controls) due to not having sufficient data.

Data exclusions We started with 47 preterm births and 94 term births, but excluded 2 preterms and 4 terms because we lacked the $16 \mathrm{~S}$ rRNA taxonomic data.

Replication Experimental replication was not possible due to extensive cost. We attempted to replicate our findings using other data sets, with significant but not ideal results (see the manuscript). Sufficient data are not available in the community for such replication studies.

Randomization All samples were randomized for sequencing and cytokine assay experiments. For $16 \mathrm{~S}$ rRNA data, samples were randomized at the PCR stage and again at the sequencing stage.

Blinding

Case matching was performed blinded to all other study data.

\section{Reporting for specific materials, systems and methods}

We require information from authors about some types of materials, experimental systems and methods used in many studies. Here, indicate whether each material, system or method listed is relevant to your study. If you are not sure if a list item applies to your research, read the appropriate section before selecting a response.

Materials \& experimental systems

$\mathrm{n} / \mathrm{a}$ Involved in the study

\ $\square$ Antibodies

Х Eukaryotic cell lines

\ $\square$ Palaeontology

\ $\square$ Animals and other organisms

$\square \bigotimes$ Human research participants

$\bigotimes \mid \square$ Clinical data

\section{Methods}

n/a Involved in the study

$\bigotimes \square$ ChIP-seq

$\bigotimes \square$ Flow cytometry

$\triangle \square$ MRI-based neuroimaging 
Policy information about studies involving human research participants

Population characteristics The population includes pregnant women 15 years and older and their neonates. Women selected for the the MOMS-PI Preterm study were predominantly of African ancestry ( $78 \%$ ) with a household income of less than $\$ 20,000$ annually $(76 \%)$.

Recruitment

Participants for this study were enrolled from women visiting maternity clinics in Virginia and Washington State. All study procedures involving human subjects were reviewed and approved by the institutional review board at Virginia Commonwealth University (IRB\# HM15527). Participants were enrolled at multiple sites in Washington State by our partner registry, the Global Alliance to Prevent Prematurity and Stillbirth (GAPPS, www.gapps.org/). Study protocols were harmonized across sites, and data and samples from participants enrolled in Washington State were distributed to the VCU site. All study participants enrolled in Virginia and most participants enrolled at Washington State sites were also enrolled in the Research Alliance for Microbiome 3

nature research | reporting summary April 2018

Science (RAMS) Registry at Virginia Commonwealth University. RAMS Registry protocols were approved at Virginia Commonwealth University (IRB\# HM15528); GAPPS-associated sites ceded review to the VCU IRB through reliance agreements. The study was performed with compliance to all relevant ethical regulations. Written informed consent was obtained from all participants and parental permission and assent was obtained for participating minors at least 15 years of age.

Pregnant women were provided literature on the project and invited to participate in the study. Women who: i) were incapable of understanding the informed consent or assent forms, or ii) were incarcerated were excluded from the study. Comprehensive demographic, health history and dietary assessment surveys were administered, and relevant clinical data (e.g., gestational age, height, weight, blood pressure, vaginal pH, diagnosis, etc.) was recorded. Relevant clinical information was also obtained from neonates at birth and discharge.

At subsequent prenatal visits, triage, in labor and delivery, and at discharge, additional surveys were administered, relevant clinical data was recorded and samples were collected. Vaginal and rectal samples were not collected at labor and delivery or at discharge. Women with any of the following conditions were excluded from sampling at a given visit:

1. Incapable of self-sampling due to mental, emotional or physical limitations.

2. More than minimal vaginal bleeding as judged by the clinician.

3. Ruptured membranes prior to 37 weeks.

4. Active herpes lesions in the vulvovaginal region.

Case/control design. We selected 47 preterm cases of singleton, non-medically indicated preterm births from women who delivered between 23 weeks 1 day and 36 weeks 6 days gestation and were enrolled in the Virginia arm of the study and delivered at the site prior to August 2016. From this cohort of 627 women, 82 delivered prior to 37 weeks. Tweleve of the participants who delivered preterm had multiple gestation pregancies, 21 experienced medically indicated delivery, one delivered following fetal demise and one delivered a fetus at a non-viable gestational age. The participants had completed the study through delivery, and their gestational age information had been recorded in the study operational database as of July 2016. We case-matched the preterm participants 2:1 with participants who completed the study with singleton term deliveries $\geq 39$ weeks to avoid complications associated with early term birth51-53, matching based on ethnicity, age and income. With these criteria, we matched controls to cases as close as possible, loosening criteria at each pass using an in-house script; a few difficult-to-match cases were matched by hand. Case matching was performed blinded to all other study data. Two of the 47 preterm births did not have $16 \mathrm{~S}$ rRNA that passed QC, thus these PTB samples and their controls were excluded from the taxonomic 16S rRNA analyses (Fig. 1) and demographic data in Table 1.

Possible self-selection bias. Women were approached in our prenatal clinics, informed of the project with its goals and protocols, and asked if they would like to participate. Since women were given the choice to participate, there is always the possibility that there could be self selection bias. That said, we detected no such bias, as most women were motivated to participate as they were quite aware of the challenges of adverse events in pregnancy and preterm birth.

Ethics oversight Institutional Review Board for Human Subjects REsearch at VCU 